\title{
GRADED DECOMPOSITION MATRICES OF $v$-SCHUR ALGEBRAS VIA JANTZEN FILTRATION
}

\author{
PENG SHAN
}

\begin{abstract}
We prove that certain parabolic Kazhdan-Lusztig polynomials calculate the graded decomposition matrices of $v$-Schur algebras given by the Jantzen filtration of Weyl modules, confirming a conjecture of Leclerc and Thibon.
\end{abstract}

\section{Contents}

Introduction

1. Jantzen filtration of standard modules

2. Affine parabolic category $\mathcal{O}$ and $v$-Schur algebras

3. Generalities on $\mathscr{D}$-modules on ind-schemes

4. Localization theorem for affine Lie algebras of negative level

5. The geometric construction of the Jantzen filtration

6. Proof of the main theorem

Appendix A. Kashiwara-Tanisaki's construction, translation functors and Proof of Proposition 4.2

Appendix B. Proof of Proposition 5.4

Index of notation

Acknowledgement

References

\section{INTRODUCTION}

Let $v$ be a $r$-th root of unity in $\mathbb{C}$. The $v$-Schur algebra $\mathbf{S}_{v}(n)$ over $\mathbb{C}$ is a finite dimensional quasi-hereditary algebra. Its standard modules are the Weyl modules $W_{v}(\lambda)$ indexed by partitions $\lambda$ of $n$. The module $W_{v}(\lambda)$ has a simple quotient $L_{v}(\lambda)$. See Section 2.9 for more details.

The decomposition matrix of $\mathbf{S}_{v}(n)$ is given by the following algorithm. Let $\mathcal{F}_{q}$ be the Fock space of level one. It is a $\mathbb{Q}(q)$-vector space with a basis $\{|\lambda\rangle\}$ indexed by the set of partitions. Moreover, it carries an action of the quantum enveloping algebra $U_{q}\left(\widehat{\mathfrak{s l}}_{r}\right)$. Let $L^{+}$(resp. $L^{-}$) be the $\mathbb{Z}[q]$-submodule (resp. $\mathbb{Z}\left[q^{-1}\right]$ submodule) in $\mathcal{F}_{q}$ spanned by $\{|\lambda\rangle\}$. Following Leclerc and Thibon [LT1, Theorem 4.1], the Fock space $\mathcal{F}_{q}$ admits two particular bases $\left\{G_{\lambda}^{+}\right\},\left\{G_{\lambda}^{-}\right\}$with the properties that

$$
G_{\lambda}^{+} \equiv|\lambda\rangle \bmod q L^{+}, \quad G_{\lambda}^{-} \equiv|\lambda\rangle \bmod q^{-1} L^{-} .
$$

Received by the editors March 27, 2011.

2010 Mathematics Subject Classification. Primary 20G43. 
Let $d_{\lambda \mu}(q), e_{\lambda \mu}(q)$ be the elements in $\mathbb{Z}[q]$ such that

$$
G_{\mu}^{+}=\sum_{\lambda} d_{\lambda \mu}(q)|\lambda\rangle, \quad G_{\lambda}^{-}=\sum_{\mu} e_{\lambda \mu}\left(-q^{-1}\right)|\mu\rangle .
$$

For any partition $\lambda$ write $\lambda^{\prime}$ for the transposed partition. Then the Jordan-Hölder multiplicity of $L_{v}(\mu)$ in $W_{v}(\lambda)$ is equal to the value of $d_{\lambda^{\prime} \mu^{\prime}}(q)$ at $q=1$. This result was conjectured by Leclerc and Thibon [LT1, Conjecture 5.2] and has been proved by Varagnolo and Vasserot VV1.

We are interested in the Jantzen filtration of $W_{v}(\lambda)[\mathrm{JM}]$ :

$$
W_{v}(\lambda)=J^{0} W_{v}(\lambda) \supset J^{1} W_{v}(\lambda) \supset \ldots
$$

It is a filtration by $\mathbf{S}_{v}(n)$-submodules. The graded decomposition matrix of $\mathbf{S}_{v}(n)$ counts the multiplicities of $L_{v}(\mu)$ in the associated graded module of $W_{v}(\lambda)$. The graded version of the above algorithm was also conjectured by Leclerc and Thibon [LT1, Conjecture 5.3]. Our main result is a proof of this conjecture under a mild restriction on $v$.

Theorem 0.1. Suppose that $v=\exp (2 \pi i / \kappa)$ with $\kappa \in \mathbb{Z}$ and $\kappa \leqslant-3$. Let $\lambda, \mu$ be partitions of $n$. Then

$$
d_{\lambda^{\prime} \mu^{\prime}}(q)=\sum_{i \geqslant 0}\left[J^{i} W_{v}(\lambda) / J^{i+1} W_{v}(\lambda): L_{v}(\mu)\right] q^{i} .
$$

Let us outline the idea of the proof. We first show that certain equivalence of highest weight categories preserves the Jantzen filtrations of standard modules (Proposition 1.8). By constructing such an equivalence between the module category of the $v$-Schur algebra and a subcategory of the affine parabolic category $\mathcal{O}$ of negative level, we then transfer the problem of computing the Jantzen filtration of Weyl modules into the same problem for parabolic Verma modules (Corollary 2.14). The latter is solved using Beilinson-Bernstein's techniques (Sections 4, 5, 6).

\section{JANTZEN FILTRATION OF STANDARD MODULES}

1.1. Notation. We will denote by $A$-mod the category of finitely generated modules over an algebra $A$, and by $A$-proj its subcategory consisting of projective objects. Let $R$ be a commutative noetherian $\mathbb{C}$-algebra. By a finite projective $R$-algebra we mean an $R$-algebra that belongs to $R$-proj.

A $R$-category $\mathcal{C}$ is a category whose Hom sets are $R$-modules. All the functors between $R$-categories will be assumed to be $R$-linear, i.e., they induce morphisms of $R$-modules on the Hom sets. Unless otherwise specified, all the functors will be assumed to be covariant. If $\mathcal{C}$ is abelian, we will write $\mathcal{C}$-proj for the full subcategory consisting of projective objects. If there exists a finite projective algebra $A$ together with an equivalence of $R$-categories $F: \mathcal{C} \cong A$-mod, then we define $\mathcal{C} \cap R$-proj to be the full subcategory of $\mathcal{C}$ consisting of objects $M$ such that $F(M)$ belongs to $R$-proj. By Morita theory, the definition of $\mathcal{C} \cap R$-proj is independent of $A$ or $F$. Further, for any $\mathbb{C}$-algebra homomorphism $R \rightarrow R^{\prime}$ we will abbreviate $R^{\prime} \mathcal{C}$ for the category $\left(R^{\prime} \otimes_{R} A\right)$-mod. The definition of $R^{\prime} \mathcal{C}$ is independent of the choice of $A$ up to equivalence of categories.

For any abelian category $\mathcal{C}$ we will write $[\mathcal{C}]$ for the Grothendieck group of $\mathcal{C}$. Any exact functor $F$ from $\mathcal{C}$ to another abelian category $\mathcal{C}^{\prime}$ yields a group homomorphism $[\mathcal{C}] \rightarrow\left[\mathcal{C}^{\prime}\right]$, which we will again denote by $F$. 
A $\mathbb{C}$-category $\mathcal{C}$ is called artinian if the Hom sets are finite dimensional $\mathbb{C}$-vector spaces and every object has a finite length. The Jordan-Hölder multiplicity of a simple object $L$ in an object $M$ of $\mathcal{C}$ will be denoted by $[M: L]$.

We abbreviate $\otimes=\otimes_{\mathbb{C}}$ and Hom $=$ Hom $_{\mathbb{C}}$.

1.2. Highest weight categories. Let $\mathcal{C}$ be a $R$-category that is equivalent to the category $A$-mod for some finite projective $R$-algebra $A$. Let $\Delta$ be a finite set of objects of $\mathcal{C}$ together with a partial order $<$. Let $\mathcal{C}^{\Delta}$ be the full subcategory of $\mathcal{C}$ consisting of objects which admit a finite filtration such that the successive quotients are isomorphic to objects in

$$
\{D \otimes U \mid D \in \Delta, U \in R \text {-proj }\} .
$$

We have the following definition; see [R, Definition 4.11].

Definition 1.1. The pair $(\mathcal{C}, \Delta)$ is called a highest weight $R$-category if the following conditions hold:

- the objects of $\Delta$ are projective over $R$,

- we have $\operatorname{End}_{\mathcal{C}}(D)=R$ for all $D \in \Delta$,

- given $D_{1}, D_{2} \in \Delta$, if $\operatorname{Hom}_{\mathcal{C}}\left(D_{1}, D_{2}\right) \neq 0$, then $D_{1} \leqslant D_{2}$,

- if $M \in \mathcal{C}$ satisfies $\operatorname{Hom}_{\mathcal{C}}(D, M)=0$ for all $D \in \Delta$, then $M=0$,

- given $D \in \Delta$, there exists $P \in \mathcal{C}$-proj and a surjective morphism $f: P \rightarrow D$ such that ker $f$ belongs to $\mathcal{C}^{\Delta}$. Moreover, in the filtration of ker $f$ only $D^{\prime} \otimes U$ with $D^{\prime}>D$ appears.

The objects in $\Delta$ are called standard. We say that an object has a standard filtration if it belongs to $\mathcal{C}^{\Delta}$. There is another set $\nabla$ of objects in $\mathcal{C}$, called costandard objects, given by the following proposition.

Proposition 1.2. Let $(\mathcal{C}, \Delta)$ be a highest weight $R$-category. Then there is a set $\nabla=\left\{D^{\vee} \mid D \in \Delta\right\}$ of objects of $\mathcal{C}$, unique up to isomorphism, with the following properties:

(a) the pair $\left(\mathcal{C}^{\mathrm{op}}, \nabla\right)$ is a highest weight $R$-category, where $\nabla$ is equipped with the same partial order as $\Delta$,

(b) for $D_{1}, D_{2} \in \Delta$ we have $\operatorname{Ext}_{\mathcal{C}}^{i}\left(D_{1}, D_{2}^{\vee}\right) \cong \begin{cases}R & \text { if } i=0 \text { and } D_{1}=D_{2}, \\ 0 & \text { else. }\end{cases}$

See [R, Proposition 4.19].

1.3. Base change for highest weight categories. From now on, unless otherwise specified we will fix $R=\mathbb{C}[[s]]$, the ring of formal power series in the variable $s$. Let $\wp$ be its maximal ideal and let $K$ be its fraction field. For any $R$-module $M$, any morphism $f$ of $R$-modules and any $i \in \mathbb{N}$ we will write

$$
\begin{aligned}
M\left(\wp^{i}\right) & =M \otimes_{R}\left(R / \wp^{i} R\right), & & M_{K}=M \otimes_{R} K, \\
f\left(\wp^{i}\right) & =f \otimes_{R}\left(R / \wp^{i} R\right), & & f_{K}=f \otimes_{R} K .
\end{aligned}
$$

We will abbreviate

$$
\mathcal{C}(\wp)=R(\wp) \mathcal{C}, \quad \mathcal{C}_{K}=K \mathcal{C} .
$$

Let us first recall the following basic facts. 
Lemma 1.3. Let $A$ be a finite projective $R$-algebra. Let $P \in A$-mod.

(a) The $A$-module $P$ is projective if and only if $P$ is a projective $R$-module and $P(\wp)$ belongs to $A(\wp)$-proj.

(b) If $P$ belongs to $A$-proj, then we have a canonical isomorphism

$$
\operatorname{Hom}_{A}(P, M)(\wp) \stackrel{\sim}{\rightarrow} \operatorname{Hom}_{A(\wp)}(P(\wp), M(\wp)), \quad \forall M \in A-\bmod .
$$

Further, if $M$ belongs to $R$-proj, then $\operatorname{Hom}_{A}(P, M)$ also belongs to $R$-proj.

We will also need the following theorem of Rouquier [R, Theorem 4.15].

Proposition 1.4. Let $\mathcal{C}$ be a $R$-category that is equivalent to $A$-mod for some finite projective $R$-algebra $A$. Let $\Delta$ be a finite poset of objects of $\mathcal{C} \cap R$-proj. Then the category $(\mathcal{C}, \Delta)$ is a highest weight $R$-category if and only if $(\mathcal{C}(\wp), \Delta(\wp))$ is a highest weight $\mathbb{C}$-category.

Finally, the costandard objects can also be characterized in the following way.

Lemma 1.5. Let $(\mathcal{C}, \Delta)$ be a highest weight $R$-category. Assume that $\nabla^{\prime}=\left\{{ }^{\vee} D \mid D \in\right.$ $\Delta\}$ is a set of objects of $\mathcal{C} \cap R$-proj such that for any $D \in \Delta$ we have

$$
\left({ }^{\vee} D\right)(\wp) \cong D(\wp)^{\vee}, \quad\left({ }^{\vee} D\right)_{K} \cong D_{K} .
$$

Then we have ${ }^{\vee} D \cong D^{\vee} \in \nabla$.

Proof. We prove the lemma by showing that $\nabla^{\prime}$ has the properties (a), (b) in Proposition 1.2 with ${ }^{\vee} D$ playing the role of $D^{\vee}$. This will imply that ${ }^{\vee} D \cong D^{\vee} \in \nabla$. To check (a) note that $\nabla^{\prime}(\wp)$ is the set of costandard modules of $\mathcal{C}(\wp)$ by assumption. So $\left(\mathcal{C}(\wp)^{\text {op }}, \nabla^{\prime}(\wp)\right)$ is a highest weight $\mathbb{C}$-category. Therefore $\left(\mathcal{C}^{\text {op }}, \nabla^{\prime}\right)$ is a highest weight $R$-category by Proposition [1.4. Now, let us concentrate on (b). Given $D_{1}$, $D_{2} \in \Delta$, let $P_{\bullet}=0 \rightarrow P_{n} \rightarrow \cdots \rightarrow P_{0}$ be a projective resolution of $D_{1}$ in $\mathcal{C}$. Then $\operatorname{Ext}_{\mathcal{C}}^{i}\left(D_{1},{ }^{\vee} D_{2}\right)$ is the cohomology of the complex

$$
C_{\bullet}=\operatorname{Hom}_{\mathcal{C}}\left(P_{\bullet},{ }^{\vee} D_{2}\right) .
$$

Since $D_{1}$ and all the $P_{i}$ belong to $R$-proj and $R$ is a discrete valuation ring, by the Universal Coefficient Theorem the complex

$$
P_{\bullet}(\wp)=0 \rightarrow P_{n}(\wp) \rightarrow \cdots \rightarrow P_{0}(\wp)
$$

is a resolution of $D_{1}(\wp)$ in $\mathcal{C}(\wp)$. Further, each $P_{i}(\wp)$ is a projective object in $\mathcal{C}(\wp)$ by Lemma 1.3(a). So $\operatorname{Ext}_{\mathcal{C}(\wp)}^{i}\left(D_{1}(\wp),{ }^{\vee} D_{2}(\wp)\right)$ is given by the cohomology of the complex

$$
C \bullet(\wp)=\operatorname{Hom}_{\mathcal{C}(\wp)}\left(P \bullet(\wp),{ }^{\vee} D_{2}(\wp)\right) .
$$

Again, by the Universal Coefficient Theorem, the canonical map

$$
H_{i}\left(C_{\bullet}\right)(\wp) \longrightarrow H_{i}(C(\wp) \bullet)
$$

is injective. In other words, we have a canonical injective map

$$
\operatorname{Ext}_{\mathcal{C}}^{i}\left(D_{1},{ }^{\vee} D_{2}\right)(\wp) \longrightarrow \operatorname{Ext}_{\mathcal{C}(\wp)}^{i}\left(D_{1}(\wp),{ }^{\vee} D_{2}(\wp)\right) .
$$

Note that each $R$-module $C_{i}$ is finitely generated. Therefore $\operatorname{Ext}_{\mathcal{C}}^{i}\left(D_{1},{ }^{\vee} D_{2}\right)$ is also finitely generated over $R$. Note that if $i>0$, or $i=0$ and $D_{1} \neq D_{2}$, then the right hand side of (1.1) is zero by assumption. So $\operatorname{Ext}_{\mathcal{C}}^{i}\left(D_{1},{ }^{\vee} D_{2}\right)(\wp)=0$, and 
hence $\operatorname{Ext}_{\mathcal{C}}^{i}\left(D_{1},{ }^{\vee} D_{2}\right)=0$ by Nakayama's lemma. Now, let us concentrate on the $R$-module $\operatorname{Hom}_{\mathcal{C}}\left(D,{ }^{\vee} D\right)$ for $D \in \Delta$. First, we have

$$
\begin{aligned}
\operatorname{Hom}_{\mathcal{C}}\left(D,{ }^{\vee} D\right) \otimes_{R} K & =\operatorname{Hom}_{\mathcal{C}_{K}}\left(D_{K},\left({ }^{\vee} D\right)_{K}\right) \\
& =\operatorname{End}_{\mathcal{C}_{K}}\left(D_{K}\right) \\
& =\operatorname{End}_{\mathcal{C}}(D) \otimes_{R} K \\
& =K .
\end{aligned}
$$

Here the second equality is given by the isomorphism $D_{K} \cong\left({ }^{\vee} D\right)_{K}$ and the last equality follows from $\operatorname{End}_{\mathcal{C}}(D)=R$. Next, note that $\operatorname{Hom}_{\mathcal{C}}\left(D,{ }^{\vee} D\right)(\wp)$ is included into the vector space $\operatorname{Hom}_{\mathcal{C}(\wp)}\left(D(\wp),{ }^{\vee} D(\wp)\right)=\mathbb{C}$ by (1.1). So its dimension over $\mathbb{C}$ is less than one. Together with (1.2) this yields an isomorphism of $R$-modules $\operatorname{Hom}_{\mathcal{C}}\left(D,{ }^{\vee} D\right) \cong R$, because $R$ is a discrete valuation ring. So we have verified that $\nabla^{\prime}$ satisfies both properties (a) and (b) in Proposition 1.2. Therefore it coincides with $\nabla$ and ${ }^{\vee} D$ is isomorphic to $D^{\vee}$.

1.4. The Jantzen filtration of standard modules. Let $\left(\mathcal{C}_{\mathbb{C}}, \Delta_{\mathbb{C}}\right)$ be a highest weight $\mathbb{C}$-category and $(\mathcal{C}, \Delta)$ be a highest weight $R$-category such that $\left(\mathcal{C}_{\mathbb{C}}, \Delta_{\mathbb{C}}\right) \cong$ $(\mathcal{C}(\wp), \Delta(\wp))$. Then any standard module in $\Delta_{\mathbb{C}}$ admits a Jantzen type filtration associated with $(\mathcal{C}, \Delta)$. It is given as follows.

Definition 1.6. For any $D \in \Delta$ let $\phi: D \rightarrow D^{\vee}$ be a morphism in $\mathcal{C}$ such that $\phi(\wp) \neq 0$. For any positive integer $i$ let

$$
\pi_{i}: D^{\vee} \longrightarrow D^{\vee} / \wp^{i} D^{\vee}
$$

be the canonical quotient map. Set

$$
D^{i}=\operatorname{ker}\left(\pi_{i} \circ \phi\right) \subset D, \quad J^{i} D(\wp)=\left(D^{i}+\wp D\right) / \wp D .
$$

The Jantzen filtration of $D(\wp)$ is the filtration

$$
D(\wp)=J^{0} D(\wp) \supset J^{1} D(\wp) \supset \cdots .
$$

To see that the Jantzen filtration is well defined, one notices first that the morphism $\phi$ always exists because $\operatorname{Hom}_{\mathcal{C}}\left(D, D^{\vee}\right)(\wp) \cong R(\wp)$. Further, the filtration is independent of the choice of $\phi$. Because if $\phi^{\prime}: D \rightarrow D^{\vee}$ is another morphism such that $\phi^{\prime}(\wp) \neq 0$, the fact that $\operatorname{Hom}_{\mathcal{C}}\left(D, D^{\vee}\right) \cong R$ and $\phi(\wp) \neq 0$ implies that there exists an element $a$ in $R$ such that $\phi^{\prime}=a \phi$. Moreover, $\phi^{\prime}(\wp) \neq 0$ implies that $a$ is invertible in $R$. So $\phi$ and $\phi^{\prime}$ define the same filtration.

Remark 1.7. If the category $\mathcal{C}_{K}$ is semi-simple, then the Jantzen filtration of any standard module $D(\wp)$ is finite. In fact, $\operatorname{since}_{\operatorname{End}}(D)=R$ we have $\operatorname{End}_{\mathcal{C}_{K}}\left(D_{K}\right)=$ $K$. Therefore $D_{K}$ is an indecomposable object in $\mathcal{C}_{K}$. So the semi-simplicity of $\mathcal{C}_{K}$ implies that $D_{K}$ is simple. Similarly, $D_{K}^{\vee}$ is also simple. So the morphism $\phi_{K}: D_{K} \rightarrow D_{K}^{\vee}$ is an isomorphism. In particular, $\phi$ is injective. Now, consider the intersection

$$
\bigcap_{i} J^{i} D(\wp)=\bigcap_{i}\left(D^{i}+\wp D\right) / \wp D \text {. }
$$

Since we have $D^{i} \supset D^{i+1}$, the intersection on the right hand side is equal to $\left(\left(\bigcap_{i} D^{i}\right)+\wp D\right) / \wp D$. The injectivity of $\phi$ implies that $\bigcap_{i} D^{i}=\operatorname{ker} \phi$ is zero. Hence $\bigcap_{i} J^{i} D(\wp)=0$. Since $D(\wp) \in \mathcal{C}(\wp)$ has a finite length, we have $J^{i} D(\wp)=0$ for $i$ large enough. 
1.5. Equivalences of highest weight categories and Jantzen filtrations. Let $\left(\mathcal{C}_{1}, \Delta_{1}\right),\left(\mathcal{C}_{2}, \Delta_{2}\right)$ be highest weight $R$-categories (resp. $\mathbb{C}$-categories or $K$ categories). A functor $F: \mathcal{C}_{1} \rightarrow \mathcal{C}_{2}$ is an equivalence of highest weight categories if it is an equivalence of categories and if for any $D_{1} \in \Delta_{1}$ there exists $D_{2} \in \Delta_{2}$ such that $F\left(D_{1}\right) \cong D_{2}$. Note that for such an equivalence $F$ we also have

$$
F\left(D_{1}^{\vee}\right) \cong D_{2}^{\vee},
$$

because the two properties in Proposition 1.2 which characterize the costandard objects are preserved by $F$.

Let $F: \mathcal{C}_{1} \rightarrow \mathcal{C}_{2}$ be an exact functor. Since $\mathcal{C}_{1}$ is equivalent to $A$-mod for some finite projective $R$-algebra $A$, the functor $F$ is represented by a projective object $P$ in $\mathcal{C}_{1}$, i.e., we have $F \cong \operatorname{Hom}_{\mathcal{C}_{1}}(P,-)$. Set

$$
F(\wp)=\operatorname{Hom}_{\mathcal{C}_{1}(\wp)}(P(\wp),-): \mathcal{C}_{1}(\wp) \rightarrow \mathcal{C}_{2}(\wp) .
$$

Note that the functor $F(\wp)$ is unique up to equivalence of categories. It is an exact functor, and it is isomorphic to the functor $\operatorname{Hom}_{\mathcal{C}_{1}}(P,-)(\wp)$; see Lemma 1.3. In particular, for $D \in \Delta_{1}$ there are canonical isomorphisms

$$
F(D)(\wp) \cong F(\wp)(D(\wp)), \quad F\left(D^{\vee}\right)(\wp) \cong F(\wp)\left(D^{\vee}(\wp)\right) .
$$

Proposition 1.8. Let $\left(\mathcal{C}_{1}, \Delta_{1}\right),\left(\mathcal{C}_{2}, \Delta_{2}\right)$ be two equivalent highest weight $R$-categories. Fix an equivalence $F: \mathcal{C}_{1} \rightarrow \mathcal{C}_{2}$. Then the following holds.

(a) The functor $F(\wp)$ is an equivalence of highest weight categories.

(b) The functor $F(\wp)$ preserves the Jantzen filtration of standard modules, i.e., for any $D_{1} \in \Delta_{1}$ let $D_{2}=F\left(D_{1}\right) \in \Delta_{2}$, then

$$
F(\wp)\left(J^{i} D_{1}(\wp)\right)=J^{i} D_{2}(\wp), \quad \forall i \in \mathbb{N} .
$$

Proof. (a) If $G: \mathcal{C}_{2} \rightarrow \mathcal{C}_{1}$ is a quasi-inverse of $F$, then $G(\wp)$ is a quasi-inverse of $F(\wp)$. So $F(\wp)$ is an equivalence of categories. It maps a standard object to a standard one because of the first isomorphism in (1.5).

(b) The functor $F$ yields an isomorphism of $R$-modules

$$
\operatorname{Hom}_{\mathcal{C}_{1}}\left(D_{1}, D_{1}^{\vee}\right) \stackrel{\sim}{\rightarrow} \operatorname{Hom}_{\mathcal{C}_{2}}\left(F\left(D_{1}\right), F\left(D_{1}^{\vee}\right)\right),
$$

where the right hand side identifies with $\operatorname{Hom}_{\mathcal{C}_{2}}\left(D_{2}, D_{2}^{\vee}\right)$ via the isomorphism (1.4). Let $\phi_{1}$ be an element in $\operatorname{Hom}_{\mathcal{C}_{1}}\left(D_{1}, D_{1}^{\vee}\right)$ such that $\phi_{1}(\wp) \neq 0$. Let

$$
\phi_{2}=F\left(\phi_{1}\right): D_{2} \rightarrow D_{2}^{\vee} \text {. }
$$

Then we also have $\phi_{2}(\wp) \neq 0$.

For $a=1,2$ and $i \in \mathbb{N}$ let $\pi_{a, i}: D_{a}^{\vee} \rightarrow D_{a}^{\vee}\left(\wp^{i}\right)$ be the canonical quotient map. Since $F$ is $R$-linear and exact, the isomorphism $F\left(D_{1}^{\vee}\right) \cong D_{2}^{\vee}$ maps $F\left(\wp^{i} D_{1}^{\vee}\right)$ to $\wp^{i} D_{2}^{\vee}$ and induces an isomorphism

$$
F\left(D_{1}^{\vee}\left(\wp^{i}\right)\right) \cong D_{2}^{\vee}\left(\wp^{i}\right) .
$$

Under these isomorphisms the morphism $F\left(\pi_{1, i}\right)$ is identified with $\pi_{2, i}$. So we have

$$
\begin{aligned}
F\left(D_{1}^{i}\right) & =F\left(\operatorname{ker}\left(\pi_{1, i} \circ \phi_{1}\right)\right) \\
& =\operatorname{ker}\left(F\left(\pi_{1, i}\right) \circ F\left(\phi_{1}\right)\right) \\
& \cong \operatorname{ker}\left(\pi_{2, i} \circ \phi_{2}\right) \\
& =D_{2}^{i} .
\end{aligned}
$$


Now, apply $F$ to the short exact sequence

$$
0 \rightarrow \wp D_{1} \rightarrow D_{1}^{i}+\wp D_{1} \rightarrow J^{i} D_{1}(\wp) \rightarrow 0,
$$

we get

$$
\begin{aligned}
F\left(J^{i} D_{1}(\wp)\right) & \cong\left(F\left(D_{1}^{i}\right)+\wp F\left(D_{1}\right)\right) / \wp F\left(D_{1}\right) \\
& \cong J^{i} D_{2}(\wp) .
\end{aligned}
$$

Since $F\left(J^{i} D_{1}(\wp)\right)=F(\wp)\left(J^{i} D_{1}(\wp)\right)$, the proposition is proved.

\section{Affine parabolic CATEgory $\mathcal{O}$ And $v$-Schur algebras}

2.1. The affine Lie algebra. Fix an integer $m>1$. Let $G_{0} \supset B_{0} \supset T_{0}$ be respectively the linear algebraic group $G L_{m}(\mathbb{C})$, the Borel subgroup of upper triangular matrices and the maximal torus of diagonal matrices. Let $\mathfrak{g}_{0} \supset \mathfrak{b}_{0} \supset \mathfrak{t}_{0}$ be their Lie algebras. Let

$$
\mathfrak{g}=\mathfrak{g}_{0} \otimes \mathbb{C}\left[t, t^{-1}\right] \oplus \mathbb{C} \mathbf{1} \oplus \mathbb{C} \partial
$$

be the affine Lie algebra of $\mathfrak{g}_{0}$. Its Lie bracket is given by $\left[\xi \otimes t^{a}+x \mathbf{1}+y \partial, \xi^{\prime} \otimes t^{b}+x^{\prime} \mathbf{1}+y^{\prime} \partial\right]=\left[\xi, \xi^{\prime}\right] \otimes t^{a+b}+a \delta_{a,-b} \operatorname{tr}\left(\xi \xi^{\prime}\right) \mathbf{1}+b y \xi^{\prime} \otimes t^{b}-a y^{\prime} \xi \otimes t^{a}$, where tr $: \mathfrak{g}_{0} \rightarrow \mathbb{C}$ is the trace map. Set $\mathfrak{t}=\mathfrak{t}_{0} \oplus \mathbb{C} \mathbf{1} \oplus \mathbb{C} \partial$.

For any Lie algebra $\mathfrak{a}$ over $\mathbb{C}$, let $\mathcal{U}(\mathfrak{a})$ be its enveloping algebra. For any $\mathbb{C}$ algebra $R$, we will abbreviate $\mathfrak{a}_{R}=\mathfrak{a} \otimes R$ and $\mathcal{U}\left(\mathfrak{a}_{R}\right)=\mathcal{U}(\mathfrak{a}) \otimes R$.

In the rest of the paper, we will fix once for all an integer $c$ such that

$$
\kappa=c+m \in \mathbb{Z}_{<0} .
$$

Let $\mathcal{U}_{\kappa}$ be the quotient of $\mathcal{U}(\mathfrak{g})$ by the two-sided ideal generated by $\mathbf{1}-c$. The $\mathcal{U}_{\kappa}$-modules are precisely the $\mathfrak{g}$-modules of level $c$.

Given a $\mathbb{C}$-linear map $\lambda: \mathfrak{t} \rightarrow R$ and a $\mathfrak{g}_{R}$-module $M$ we set

$$
M_{\lambda}=\{v \in M \mid h v=\lambda(h) v, \forall h \in \mathfrak{t}\} .
$$

Whenever $M_{\lambda}$ is nonzero, we call $\lambda$ a weight of $M$.

We equip $\mathfrak{t}^{*}=\operatorname{Hom}_{\mathbb{C}}(\mathfrak{t}, \mathbb{C})$ with the basis $\epsilon_{1}, \ldots, \epsilon_{m}, \omega_{0}, \delta$ such that $\epsilon_{1}, \ldots, \epsilon_{m} \in$ $\mathfrak{t}_{0}^{*}$ is dual to the canonical basis of $\mathfrak{t}_{0}$,

$$
\delta(\partial)=\omega_{0}(\mathbf{1})=1, \quad \omega_{0}\left(\mathfrak{t}_{0} \oplus \mathbb{C} \partial\right)=\delta\left(\mathfrak{t}_{0} \oplus \mathbb{C} \mathbf{1}\right)=0 .
$$

Let $\langle\bullet: \bullet\rangle$ be the symmetric bilinear form on $\mathfrak{t}^{*}$ such that

$$
\left\langle\epsilon_{i}: \epsilon_{j}\right\rangle=\delta_{i j}, \quad\left\langle\omega_{0}: \delta\right\rangle=1, \quad\left\langle\mathfrak{t}_{0}^{*} \oplus \mathbb{C} \delta: \delta\right\rangle=\left\langle\mathfrak{t}_{0}^{*} \oplus \mathbb{C} \omega_{0}: \omega_{0}\right\rangle=0 .
$$

For $h \in \mathfrak{t}^{*}$ we will write $\|h\|^{2}=\langle h: h\rangle$. The weights of a $\mathcal{U}_{\kappa}$-module belong to

$$
\kappa^{*} \mathfrak{t}^{*}=\left\{\lambda \in \mathfrak{t}^{*} \mid\langle\lambda: \delta\rangle=c\right\} .
$$

Let $a$ denote the projection from $\mathfrak{t}^{*}$ to $\mathfrak{t}_{0}^{*}$. Consider the map

$$
z: \mathfrak{t}^{*} \rightarrow \mathbb{C}
$$

such that $\lambda \mapsto z(\lambda) \delta$ is the projection $\mathfrak{t}^{*} \rightarrow \mathbb{C} \delta$.

Let $\Pi$ be the root system of $\mathfrak{g}$ with simple roots $\alpha_{i}=\epsilon_{i}-\epsilon_{i+1}$ for $1 \leqslant i \leqslant m-1$ and $\alpha_{0}=\delta-\sum_{i=1}^{m-1} \alpha_{i}$. The root system $\Pi_{0}$ of $\mathfrak{g}_{0}$ is the root subsystem of $\Pi$ generated by $\alpha_{1}, \ldots, \alpha_{m-1}$. We will write $\Pi^{+}, \Pi_{0}^{+}$for the sets of positive roots in $\Pi, \Pi_{0}$, respectively.

The affine Weyl group $\mathfrak{S}$ is a Coxeter group with simple reflections $s_{i}$ for $0 \leqslant$ $i \leqslant m-1$. It is isomorphic to the semi-direct product of the symmetric group $\mathfrak{S}_{0}$ 
with the lattice $\mathbb{Z} \Pi_{0}$. There is a linear action of $\mathfrak{S}$ on $\mathfrak{t}^{*}$ such that $\mathfrak{S}_{0}$ fixes $\omega_{0}, \delta$, and acts on $\mathfrak{t}_{0}^{*}$ by permuting $\epsilon_{i}$ 's, and an element $\tau \in \mathbb{Z} \Pi_{0}$ acts by

$$
\tau(\delta)=\delta, \quad \tau\left(\omega_{0}\right)=\tau+\omega_{0}-\langle\tau: \tau\rangle \delta / 2, \quad \tau(\lambda)=\lambda-\langle\tau: \lambda\rangle \delta, \quad \forall \lambda \in \mathfrak{t}_{0}^{*} .
$$

Let $\rho_{0}$ be the half sum of positive roots in $\Pi_{0}$ and $\rho=\rho_{0}+m \omega_{0}$. The dot action of $\mathfrak{S}$ on $\mathfrak{t}^{*}$ is given by $w \cdot \lambda=w(\lambda+\rho)-\rho$. For $\lambda \in \mathfrak{t}^{*}$ we will denote by $\mathfrak{S}(\lambda)$ the stabilizer of $\lambda$ in $\mathfrak{S}$ under the dot action. Let $l: \mathfrak{S} \rightarrow \mathbb{N}$ be the length function.

2.2. The parabolic Verma modules and their deformations. The subset $\Pi_{0}$ of $\Pi$ defines a standard parabolic Lie subalgebra of $\mathfrak{g}$, which is given by

$$
\mathfrak{q}=\mathfrak{g}_{0} \otimes \mathbb{C}[t] \oplus \mathbb{C} \mathbf{1} \oplus \mathbb{C} \partial .
$$

It has a Levi subalgebra

$$
\mathfrak{l}=\mathfrak{g}_{0} \oplus \mathbb{C} \mathbf{1} \oplus \mathbb{C} \partial .
$$

The parabolic Verma modules of $\mathcal{U}_{\kappa}$ associated with $\mathfrak{q}$ are given as follows. Let $\lambda$ be an element in

$$
\Lambda^{+}=\left\{\lambda \in \kappa^{\kappa^{*}} \mid\langle\lambda: \alpha\rangle \in \mathbb{N}, \forall \alpha \in \Pi_{0}^{+}\right\} .
$$

Then there is a unique finite dimensional simple $\mathfrak{g}_{0}$-module $V(\lambda)$ of highest weight $a(\lambda)$. It can be regarded as a $\mathfrak{l}$-module by letting $h \in \mathbb{C} \mathbf{1} \oplus \mathbb{C} \partial$ act by the scalar $\lambda(h)$. It is, furthermore, a $\mathfrak{q}$-module if we let the nilpotent radical of $\mathfrak{q}$ act trivially. The parabolic Verma module of highest weight $\lambda$ is given by

$$
M_{\kappa}(\lambda)=\mathcal{U}(\mathfrak{g}) \otimes_{\mathcal{U}(\mathfrak{q})} V(\lambda) .
$$

It has a unique simple quotient, which we denote by $L_{\kappa}(\lambda)$.

Recall that $R=\mathbb{C}[[s]]$ and $\wp$ is its maximal ideal. Set

$$
\mathbf{c}=c+s \quad \text { and } \quad \mathbf{k}=\kappa+s .
$$

They are elements in $R$. Write $\mathcal{U}_{\mathbf{k}}$ for the quotient of $\mathcal{U}\left(\mathfrak{g}_{R}\right)$ by the two-sided ideal generated by $\mathbf{1}-\mathbf{c}$. So if $M$ is a $\mathcal{U}_{\mathbf{k}}$-module, then $M(\wp)$ is a $\mathcal{U}_{\kappa}$-module. Now, note that $R$ admits a $\mathfrak{q}_{R}$-action such that $\mathfrak{g}_{0} \otimes \mathbb{C}[t]$ acts trivially and $\mathfrak{t}$ acts by the weight $s \omega_{0}$. Denote this $\mathfrak{q}_{R^{-}}$-module by $R_{s \omega_{0}}$. For $\lambda \in \Lambda^{+}$the deformed parabolic Verma module $M_{\mathbf{k}}(\lambda)$ is the $\mathfrak{g}_{R}$-module induced from the $\mathfrak{q}_{R}$-module $V(\lambda) \otimes R_{s \omega_{0}}$. It is a $\mathcal{U}_{\mathbf{k}}$-module of highest weight $\lambda+s \omega_{0}$, and we have a canonical isomorphism

$$
M_{\mathbf{k}}(\lambda)(\wp) \cong M_{\kappa}(\lambda)
$$

We will abbreviate $\lambda_{s}=\lambda+s \omega_{0}$ and will write

$$
\mathbf{k}^{\mathbf{t}^{*}}=\left\{\lambda_{s} \mid \lambda \in \kappa_{\kappa} \mathbf{t}^{*}\right\} .
$$

Lemma 2.1. The $\mathfrak{g}_{K}$-module $M_{\mathbf{k}}(\lambda)_{K}=M_{\mathbf{k}}(\lambda) \otimes_{R} K$ is simple.

Proof. Assume that $M_{\mathbf{k}}(\lambda)_{K}$ is not simple. Then it contains a nontrivial submodule. This submodule must have a highest weight vector of weight $\mu_{s}$ for some $\mu \in \Lambda^{+}, \mu \neq \lambda$. By the linkage principle, there exists $w \in \mathfrak{S}$ such that $\mu_{s}=w \cdot \lambda_{s}$. Therefore $w$ fixes $\omega_{0}$, so it belongs to $\mathfrak{S}_{0}$. But then we must have $w=1$, because $\lambda, \mu \in \Lambda^{+}$. So $\lambda=\mu$. This is a contradiction. 
2.3. The Jantzen filtration of parabolic Verma modules. For $\lambda \in \Lambda^{+}$the Jantzen filtration of $M_{\kappa}(\lambda)$ is given as follows. Let $\sigma$ be the $R$-linear anti-involution on $\mathfrak{g}_{R}$ such that

$$
\sigma\left(\xi \otimes t^{n}\right)={ }^{t} \xi \otimes t^{-n}, \quad \sigma(\mathbf{1})=\mathbf{1}, \quad \sigma(\partial)=\partial .
$$

Here $\xi \in \mathfrak{g}_{0}$ and ${ }^{t} \xi$ is the transposed matrix. Let $\mathfrak{g}_{R}$ act on $\operatorname{Hom}_{R}\left(M_{\mathbf{k}}(\lambda), R\right)$ via $(x f)(v)=f(\sigma(x) v)$ for $x \in \mathfrak{g}_{R}, v \in M_{\mathbf{k}}(\lambda)$. Then

$$
\mathbf{D} M_{\mathbf{k}}(\lambda)=\bigoplus_{\mu \in \mathbf{k}^{\mathbf{t}^{*}}} \operatorname{Hom}_{R}\left(M_{\mathbf{k}}(\lambda)_{\mu}, R\right)
$$

is a $\mathfrak{g}_{R}$-submodule of $\operatorname{Hom}_{R}\left(M_{\mathbf{k}}(\lambda), R\right)$. It is the deformed dual parabolic Verma module with highest weight $\lambda_{s}$. The $\lambda_{s}$-weight spaces of $M_{\mathbf{k}}(\lambda)$ and $\mathbf{D} M_{\mathbf{k}}(\lambda)$ are both free $R$-modules of rank one. Any isomorphism between them yields, by the universal property of Verma modules, a $\mathfrak{g}_{R}$-module morphism

$$
\phi: M_{\mathbf{k}}(\lambda) \rightarrow \mathbf{D} M_{\mathbf{k}}(\lambda)
$$

such that $\phi(\wp) \neq 0$. The Jantzen filtration $\left(J^{i} M_{\kappa}(\lambda)\right)$ of $M_{\kappa}(\lambda)$ defined by [J] is the filtration given by Definition [1.6] using the morphism $\phi$ above.

2.4. The deformed parabolic category $\mathcal{O}$. The deformed parabolic category $\mathcal{O}$, denoted by $\mathcal{O}_{\mathbf{k}}$, is the category of $\mathcal{U}_{\mathbf{k}}$-modules $M$ such that

- $M=\bigoplus_{\lambda \in \mathrm{k}^{*}} M_{\lambda}$ with $M_{\lambda} \in R$-mod,

- for any $m \in M$ the $R$-module $\mathcal{U}\left(\mathfrak{q}_{R}\right) m$ is finitely generated.

It is an abelian category and contains deformed parabolic Verma modules. Replacing $\mathbf{k}$ by $\kappa$ and $R$ by $\mathbb{C}$ we get the usual parabolic category $\mathcal{O}$, denoted $\mathcal{O}_{\kappa}$.

Recall the map $z$ in (2.3). For any integer $r$ set

$$
{ }_{\kappa_{\kappa}} t^{*}=\left\{\mu \in{ }_{\kappa} t^{*} \mid r-z(\mu) \in \mathbb{Z}_{\geqslant 0}\right\} .
$$

Define ${ }_{\mathbf{k}}^{r} \mathbf{t}^{*}$ in the same manner. Let ${ }^{r} \mathcal{O}_{\kappa}$ (resp. ${ }^{r} \mathcal{O}_{\mathbf{k}}$ ) be the Serre subcategory of $\mathcal{O}_{\kappa}$ (resp. $\mathcal{O}_{\mathbf{k}}$ ) consisting of objects $M$ such that $M_{\mu} \neq 0$ implies that $\mu$ belongs to ${ }_{\kappa}^{r} t^{*}$ (resp. ${ }_{\mathrm{k}}^{\mathrm{t}^{*}}$ ). Write ${ }^{r} \Lambda^{+}=\Lambda^{+} \cap{ }_{\kappa}^{r} \mathrm{t}^{*}$. We have the following lemma.

Lemma 2.2. (a) For any finitely generated projective object $P$ in ${ }^{r} \mathcal{O}_{\mathbf{k}}$ and any $M \in{ }^{r} \mathcal{O}_{\mathbf{k}}$ the $R$-module $\operatorname{Hom}_{r_{\mathcal{O}_{\mathbf{k}}}}(P, M)$ is finitely generated and the canonical map

$$
\operatorname{Hom}_{r_{\mathcal{O}_{\mathbf{k}}}}(P, M)(\wp) \rightarrow \operatorname{Hom}_{r \mathcal{O}_{\kappa}}(P(\wp), M(\wp))
$$

is an isomorphism. Moreover, if $M$ is free over $R$, then $\operatorname{Hom}_{{ }^{O} \mathcal{O}_{\mathbf{k}}}(P, M)$ is also free over $R$.

(b) The assignment $M \mapsto M(\wp)$ yields a functor

$$
{ }^{r} \mathcal{O}_{\mathbf{k}} \rightarrow{ }^{r} \mathcal{O}_{\kappa} .
$$

This functor gives a bijection between the isomorphism classes of simple objects and a bijection between the isomorphism classes of indecomposable projective objects.

For any $\lambda \in{ }^{r} \Lambda^{+}$there is a unique finitely generated projective cover ${ }^{r} P_{\kappa}(\lambda)$ of $L_{\kappa}(\lambda)$ in ${ }^{r} \mathcal{O}_{\kappa}$; see [RW, Lemma 4.12]. Let $L_{\mathbf{k}}(\lambda),{ }^{r} P_{\mathbf{k}}(\lambda)$ be respectively the simple object and the indecomposable projective object in ${ }^{r} \mathcal{O}_{\mathbf{k}}$ that map respectively to $L_{\kappa}(\lambda), P_{\kappa}(\lambda)$ by the bijections in Lemma 2.2(b).

Lemma 2.3. The object ${ }^{r} P_{\mathbf{k}}(\lambda)$ is, up to isomorphism, the unique finitely generated projective cover of $L_{\mathbf{k}}(\lambda)$ in ${ }^{r} \mathcal{O}_{\mathbf{k}}$. It has a filtration by deformed parabolic Verma modules. In particular, it is a free $R$-module. 
The proof of Lemmas 2.2 and 2.3 can be given by imitating [F, Section 2]. There, Fiebig proved the analogue of these results for the (nonparabolic) deformed category $\mathcal{O}$ by adapting arguments of [RW]. The proof here goes in the same way, because the parabolic case is also treated in [RW]. We left the details to the reader. Note that the deformed parabolic category $\mathcal{O}$ for reductive Lie algebras has also been studied in $\mathrm{St}$.

2.5. The highest weight category $\mathcal{E}_{\mathbf{k}}$. Fix a positive integer $n \leqslant m$. Let $\mathcal{P}_{n}$ denote the set of partitions of $n$. Recall that a partition $\lambda$ of $n$ is a sequence of integers $\lambda_{1} \geqslant \cdots \geqslant \lambda_{m} \geqslant 0$ such that $\sum_{i=1}^{m} \lambda_{i}=n$. We associate $\lambda$ with the element $\sum_{i=1}^{m} \lambda_{i} \epsilon_{i} \in \mathfrak{t}_{0}^{*}$, which we denote again by $\lambda$. We will identify $\mathcal{P}_{n}$ with a subset of $\Lambda^{+}$by the following inclusion:

$$
\mathcal{P}_{n} \rightarrow \Lambda^{+}, \quad \lambda \mapsto \lambda+c \omega_{0}-\frac{\left\langle\lambda: \lambda+2 \rho_{0}\right\rangle}{2 \kappa} \delta .
$$

We will also fix an integer $r$ large enough such that $\mathcal{P}_{n}$ is contained in ${ }^{r} \Lambda^{+}$. Equip $\Lambda^{+}$with the partial order $\preceq$ given by $\lambda \preceq \mu$ if and only if there exists $w \in \mathfrak{S}$ such that $\mu=w \cdot \lambda$ and $a(\mu)-a(\lambda) \in \mathbb{N}_{0}^{+}$. Let $\unlhd$ denote the dominance order on $\mathcal{P}_{n}$ given by

$$
\lambda \unlhd \mu \Longleftrightarrow \sum_{j=1}^{i} \lambda_{j} \leqslant \sum_{j=1}^{i} \mu_{j}, \quad \forall 1 \leqslant i \leqslant m .
$$

Note that for $\lambda, \mu \in \mathcal{P}_{n}$ we have

$$
\lambda \preceq \mu \Longrightarrow \lambda \unlhd \mu,
$$

because $\lambda \preceq \mu$ implies that $\mu-\lambda \in \mathbb{N} \Pi_{0}^{+}$, which implies that

$$
\sum_{j=1}^{i} \mu_{j}-\sum_{j=1}^{i} \lambda_{j}=\left\langle\mu-\lambda, \epsilon_{1}+\cdots+\epsilon_{i}\right\rangle \geqslant 0, \quad \forall 1 \leqslant i \leqslant m .
$$

Now consider the following subset of ${ }^{r} \Lambda^{+}$:

$$
E=\left\{\mu \in{ }^{r} \Lambda^{+} \mid \mu=w \cdot \lambda \text { for some } w \in \mathfrak{S}, \lambda \in \mathcal{P}_{n}\right\} .
$$

Lemma 2.4. The set $E$ is finite.

Proof. Since $\mathcal{P}_{n}$ is finite, it is enough to show that for each $\lambda \in \mathcal{P}_{n}$ the set $\mathfrak{S} \cdot \lambda \cap^{r} \Lambda^{+}$ is finite. Note that for $w \in \mathfrak{S}_{0}$ and $\tau \in \mathbb{Z}_{0}$ we have $z(w \tau \cdot \lambda)=z(\tau \cdot \lambda)$. By (2.4) we have

If $z(\tau \cdot \lambda) \leqslant r$, then

$$
z(\tau \cdot \lambda)=z(\lambda)-\frac{\kappa}{2}\left(\left\|\tau+\frac{\lambda+\rho}{\kappa}\right\|^{2}-\left\|\frac{\lambda+\rho}{\kappa}\right\|^{2}\right) .
$$

$$
\left\|\tau+\frac{\lambda+\rho}{\kappa}\right\|^{2} \leqslant \frac{2}{-\kappa}(r-z(\lambda))+\left\|\frac{\lambda+\rho}{\kappa}\right\|^{2} .
$$

There exists only finitely many $\tau \in \mathbb{Z} \Pi_{0}$ which satisfies this condition, hence the set $E$ is finite.

Let $\mathcal{E}_{\kappa}$ be the full subcategory of ${ }^{r} \mathcal{O}_{\kappa}$ consisting of objects $M$ such that

$$
\mu \in{ }^{r} \Lambda^{+}, \mu \notin E \quad \Longrightarrow \quad \operatorname{Hom}_{r} \mathcal{O}_{\kappa}\left({ }^{r} P_{\kappa}(\mu), M\right)=0 .
$$

Note that since ${ }^{r} P_{\kappa}(\mu)$ is projective in ${ }^{r} \mathcal{O}_{\kappa}$, an object $M \in{ }^{r} \mathcal{O}_{\kappa}$ is in $\mathcal{E}_{\kappa}$ if and only if each simple subquotient of $M$ is isomorphic to $L_{\kappa}(\mu)$ for $\mu \in E$. In particular $\mathcal{E}_{\kappa}$ is abelian and it is a Serre subcategory of ${ }^{r} \mathcal{O}_{\kappa}$. Furthermore, $\mathcal{E}_{\kappa}$ is also an artinian 
category. In fact, each object $M \in \mathcal{E}_{\kappa}$ has a finite length because $E$ is finite and for each $\mu \in E$ the multiplicity of $L_{\kappa}(\mu)$ in $M$ is finite because $\operatorname{dim}_{\mathbb{C}} M_{\mu}<\infty$. Let $\mathfrak{g}^{\prime}$ denote the Lie subalgebra of $\mathfrak{g}$ given by

$$
\mathfrak{g}^{\prime}=\mathfrak{g}_{0} \otimes \mathbb{C}\left[t, t^{-1}\right] \oplus \mathbb{C} \mathbf{1} .
$$

Forgetting the $\partial$-action yields an equivalence of categories from $\mathcal{E}_{\kappa}$ to a category of $\mathfrak{g}^{\prime}$-modules; see [So, Proposition 8.1] for details. Since $\kappa$ is negative, this category of $\mathfrak{g}^{\prime}$-modules is equal to the category studied in [KL2].

Lemma 2.5. (a) For $\lambda \in E, \mu \in{ }^{r} \Lambda^{+}$such that $\left[M_{\kappa}(\lambda): L_{\kappa}(\mu)\right] \neq 0$ we have $\mu \in E$ and $\mu \preceq \lambda$.

(b) The module ${ }^{r} P_{\kappa}(\lambda)$ admits a filtration by $\mathcal{U}_{\kappa}$-modules

$$
{ }^{r} P_{\kappa}(\lambda)=P_{0} \supset P_{1} \supset \cdots \supset P_{l}=0
$$

such that $P_{0} / P_{1}$ is isomorphic to $M_{\kappa}(\lambda)$ and $P_{i} / P_{i+1} \cong M_{\kappa}\left(\mu_{i}\right)$ for some $\mu_{i} \succ \lambda$.

(c) The category $\mathcal{E}_{\kappa}$ is a highest weight $\mathbb{C}$-category with standard objects $M_{\kappa}(\lambda)$, $\lambda \in E$. The indecomposable projective objects in $\mathcal{E}_{\kappa}$ are the modules ${ }^{r} P_{\kappa}(\lambda)$ with $\lambda \in E$.

Proof. Let $\mathbf{U}_{v}$ be the quantized enveloping algebra of $\mathfrak{g}_{0}$ with parameter $v=$ $\exp (2 \pi i / \kappa)$. Then the Kazhdan-Lusztig tensor equivalence KL2, Theorem IV.38.1] identifies $\mathcal{E}_{\kappa}$ with a full subcategory of the category of finite dimensional $\mathbf{U}_{v^{-}}$ modules. It maps the module $M_{\kappa}(\lambda)$ to the Weyl module of $\mathbf{U}_{v}$ with highest weight $a(\lambda)$. Since $v$ is a root of unity, part (a) follows from the strong linkage principle for $\mathbf{U}_{v}$; see [An, Theorem 3.1]. Part (b) follows from (a) and [KL2, Proposition I.3.9]. Finally, part (c) follows directly from parts (a), (b).

Now, let us consider the deformed version. Let $\mathcal{E}_{\mathbf{k}}$ be the full subcategory of ${ }^{r} \mathcal{O}_{\mathbf{k}}$ consisting of objects $M$ such that

$$
\mu \in{ }^{r} \Lambda^{+}, \mu \notin E \quad \Longrightarrow \quad \operatorname{Hom}_{r} \mathcal{O}_{\mathbf{k}}\left({ }^{r} P_{\mathbf{k}}(\mu), M\right)=0 .
$$

Lemma 2.6. An object $M \in{ }^{r} \mathcal{O}_{\mathbf{k}}$ belongs to $\mathcal{E}_{\mathbf{k}}$ if and only if $M(\wp)$ belongs to $\mathcal{E}_{\kappa}$. In particular $M_{\mathbf{k}}(\lambda)$ and ${ }^{r} P_{\mathbf{k}}(\lambda)$ belong to $\mathcal{E}_{\mathbf{k}}$ for $\lambda \in E$.

Proof. By Lemma 2.2. (a) for any $\mu \in{ }^{r} \Lambda^{+}$the $R$-module $\operatorname{Hom}_{r} \mathcal{O}_{\mathbf{k}}\left({ }^{r} P_{\mathbf{k}}(\mu), M\right)$ is finitely generated and we have

$$
\operatorname{Hom}_{r} \mathcal{O}_{\mathbf{k}}\left({ }^{r} P_{\mathbf{k}}(\mu), M\right)(\wp)=\operatorname{Hom}_{r} \mathcal{O}_{\kappa}\left({ }^{r} P_{\kappa}(\mu), M(\wp)\right) \text {. }
$$

Therefore $\operatorname{Hom}_{{ }^{r} \mathcal{O}_{\mathbf{k}}}\left({ }^{r} P_{\mathbf{k}}(\mu), M\right)$ is nonzero if and only if $\operatorname{Hom}_{r} \mathcal{O}_{\kappa}\left({ }^{r} P_{\kappa}(\mu), M(\wp)\right)$ is nonzero by Nakayama's lemma. So the first statement follows from the definition of $\mathcal{E}_{\mathbf{k}}$ and $\mathcal{E}_{\kappa}$. The rest follows from Lemma 2.5(c).

Let

$$
P_{\mathbf{k}}(E)=\bigoplus_{\lambda \in E}{ }^{r} P_{\mathbf{k}}(\lambda), \quad P_{\kappa}(E)=\bigoplus_{\lambda \in E}^{r} P_{\kappa}(\lambda) .
$$

We have the following corollary.

Corollary 2.7. (a) The category $\mathcal{E}_{\mathbf{k}}$ is abelian.

(b) For $M \in \mathcal{E}_{\mathbf{k}}$ there exists a positive integer $d$ and a surjective map

$$
P_{\mathbf{k}}(E)^{\oplus d} \longrightarrow M \text {. }
$$

(c) The functor $\operatorname{Hom}_{r}{ }_{\mathbf{k}}\left(P_{\mathbf{k}}(E),-\right)$ yields an equivalence of $R$-categories

$$
\mathcal{E}_{\mathbf{k}} \cong \operatorname{End}_{{ }^{\mathcal{O}_{\mathbf{k}}}}\left(P_{\mathbf{k}}(E)\right)^{\mathrm{op}}-\bmod .
$$


Proof. Let $M \in \mathcal{E}_{\mathbf{k}}, N \in{ }^{r} \mathcal{O}_{\mathbf{k}}$. First assume that $N \subset M$. For $\mu \in{ }^{r} \Lambda^{+}$if $\operatorname{Hom}_{{ } \mathcal{O}_{\mathbf{k}}}\left({ }^{r} P_{\mathbf{k}}(\mu), N\right) \neq 0$, then $\operatorname{Hom}_{{ }^{r} \mathcal{O}_{\mathbf{k}}}\left({ }^{r} P_{\mathbf{k}}(\mu), M\right) \neq 0$, so $\mu$ belongs to $E$. Hence $N$ belongs to $\mathcal{E}_{\mathbf{k}}$. Now, if $N$ is a quotient of $M$, then $N(\wp)$ is a quotient of $M(\wp)$. Since $M(\wp)$ belongs to $\mathcal{E}_{\kappa}$, we also have $N(\wp) \in \mathcal{E}_{\kappa}$. Hence $N$ belongs to $\mathcal{E}_{\mathbf{k}}$ by Lemma 2.6. This proves part (a). Let us concentrate on (b). Since $M \in \mathcal{E}_{\mathbf{k}}$ we have $M(\wp) \in \mathcal{E}_{\kappa}$. The category $\mathcal{E}_{\kappa}$ is artinian with $P_{\kappa}(E)$ a projective generator. Hence there exists a positive integer $d$ and a surjective map

$$
f: P_{\kappa}(E)^{\oplus d} \longrightarrow M(\wp) \text {. }
$$

Since $P_{\mathbf{k}}(E)^{\oplus d}$ is projective in ${ }^{r} \mathcal{O}_{\mathbf{k}}$, this map lifts to a map of $\mathcal{U}_{\mathbf{k}}$-modules $\tilde{f}$ : $P_{\mathbf{k}}(E)^{\oplus d} \rightarrow M$ such that the following diagram commutes

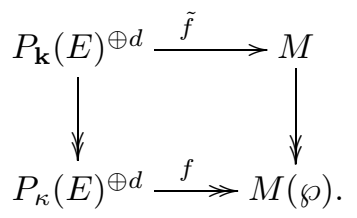

Now, since the map $\tilde{f}$ preserves weight spaces and all the weight spaces of $P_{\mathbf{k}}(E)^{\oplus r}$ and $M$ are finitely generated $R$-modules, by Nakayama's lemma, the surjectivity of $f$ implies that $\tilde{f}$ is surjective. This proves (b). Finally part (c) is a direct consequence of parts (a) and (b) by Morita theory.

Proposition 2.8. The category $\mathcal{E}_{\mathbf{k}}$ is a highest weight $R$-category with standard modules $M_{\mathbf{k}}(\mu), \mu \in E$.

Proof. Note that $\operatorname{End}_{r_{\mathcal{O}_{\mathbf{k}}}}\left(P_{\mathbf{k}}(E)\right)^{\text {op }}$ is a finite projective $R$-algebra by Lemmas 2.2 and 2.3. Since $\mathcal{E}_{\kappa}$ is a highest weight $\mathbb{C}$-category by Lemma 2.5 (c), the result follows from Proposition 1.4.

2.6. The highest weight category $\mathcal{A}_{\mathbf{k}}$. By definition $\mathcal{P}_{n}$ is a subset of $E$. Let $\mathcal{A}_{\mathbf{k}}$ be the full subcategory of $\mathcal{E}_{\mathbf{k}}$ consisting of the objects $M$ such that

$$
\operatorname{Hom}_{r} \mathcal{O}_{\mathbf{k}}\left(M_{\mathbf{k}}(\lambda), M\right)=0, \quad \forall \lambda \in E, \lambda \notin \mathcal{P}_{n} .
$$

We define the subcategory $\mathcal{A}_{\kappa}$ of $\mathcal{E}_{\kappa}$ in the same way. Let

$$
\Delta_{\mathbf{k}}=\left\{M_{\mathbf{k}}(\lambda) \mid \lambda \in \mathcal{P}_{n}\right\}, \quad \Delta_{\kappa}=\left\{M_{\kappa}(\lambda) \mid \lambda \in \mathcal{P}_{n}\right\} .
$$

Recall that $E \subset{ }^{r} \Lambda^{+}$is equipped with the partial order $\preceq$, and that $\mathcal{P}_{n} \subset E$. We have the following lemma.

Lemma 2.9. The set $\mathcal{P}_{n}$ is an ideal in $E$, i.e., for $\lambda \in E, \mu \in \mathcal{P}_{n}$, if $\lambda \preceq \mu$, then we have $\lambda \in \mathcal{P}_{n}$.

Proof. Let $\lambda \in E$ and $\mu \in \mathcal{P}_{n}$ and assume that $\lambda \preceq \mu$. Recall that $E \subset{ }_{\kappa} \mathrm{t}^{*}$, so we can write $a(\lambda)=\sum_{i=1}^{m} \lambda_{i} \epsilon_{i}$. Since $E \subset{ }^{r} \Lambda^{+}$we have $\lambda_{i} \in \mathbb{Z}$ and $\lambda_{i} \geqslant \lambda_{i+1}$. We need to show that $\lambda_{m} \in \mathbb{N}$. Since $\lambda \preceq \mu$ there exist $r_{i} \in \mathbb{N}$ such that $a(\mu)-a(\lambda)=$ $\sum_{i=1}^{m-1} r_{i} \alpha_{i}$. Therefore we have $\lambda_{m}=\mu_{m}+r_{m-1} \geqslant 0$.

Now, we can prove the following proposition.

Proposition 2.10. The category $\left(\mathcal{A}_{\mathbf{k}}, \Delta_{\mathbf{k}}\right)$ is a highest weight $R$-category with respect to the partial order $\unlhd$ on $\mathcal{P}_{n}$. The highest weight category $\left(\mathcal{A}_{\mathbf{k}}(\wp), \Delta_{\mathbf{k}}(\wp)\right)$ given by base change is equivalent to $\left(\mathcal{A}_{\kappa}, \Delta_{\kappa}\right)$. 
Proof. Since $\mathcal{E}_{\mathbf{k}}$ is a highest weight $R$-category and $\mathcal{P}_{n}$ is an ideal of $E,[\mathrm{R}$, Proposition 4.14] implies that $\left(\mathcal{A}_{\mathbf{k}}, \Delta_{\mathbf{k}}\right)$ is a highest weight $R$-category with respect to the partial order $\preceq$ on $\mathcal{P}_{n}$. By (2.7) this implies that $\left(\mathcal{A}_{\mathbf{k}}, \Delta_{\mathbf{k}}\right)$ is also a highest weight $R$-category with respect to $\unlhd$. Finally, the equivalence $\mathcal{A}_{\mathbf{k}}(\wp) \cong \mathcal{A}_{\kappa}$ follows from the equivalence $\mathcal{E}_{\mathbf{k}}(\wp) \cong \mathcal{E}_{\kappa}$ and loc. cit.

2.7. Costandard objects of $\mathcal{A}_{\mathbf{k}}$. Consider the (contravariant) duality functor $\mathbf{D}$ on $\mathcal{O}_{\mathbf{k}}$ given by

$$
\mathbf{D} M=\bigoplus_{\mu \in \mathbf{k}^{\mathbf{t}^{*}}} \operatorname{Hom}_{R}\left(M_{\mu}, R\right)
$$

where the action of $\mathcal{U}_{\mathbf{k}}$ on $\mathbf{D} M$ is given as in Section 2.3, with the module $M_{\mathbf{k}}(\lambda)$ there replaced by $M$. Similarly, we define the (contravariant) duality functor $\mathbf{D}$ on $\mathcal{O}_{\kappa}$ by

$$
\mathbf{D} M=\bigoplus_{\mu \in \mathbf{k}^{\mathbf{t}^{*}}} \operatorname{Hom}\left(M_{\mu}, \mathbb{C}\right),
$$

with the $\mathcal{U}_{\kappa}$-action given in the same way. This functor fixes the simple modules in $\mathcal{O}_{\kappa}$. Hence it restricts to a duality functor on $\mathcal{A}_{\kappa}$, because $\mathcal{A}_{\kappa}$ is a Serre subcategory of $\mathcal{O}_{\kappa}$. Therefore $\left(\mathcal{A}_{\kappa}, \Delta_{\kappa}\right)$ is a highest weight category with duality in the sense of CPS. It follows from [CPS, Proposition 1.2] that the costandard module $M_{\kappa}(\lambda)^{\vee}$ in $\mathcal{A}_{\kappa}$ is isomorphic to $\mathbf{D} M_{\kappa}(\lambda)$.

Lemma 2.11. The costandard module $M_{\mathbf{k}}(\lambda)^{\vee}$ in $\mathcal{A}_{\mathbf{k}}$ is isomorphic to $\mathbf{D} M_{\mathbf{k}}(\lambda)$ for any $\lambda \in \mathcal{P}_{n}$.

Proof. By definition we have a canonical isomorphism

$$
\left(\mathbf{D} M_{\mathbf{k}}(\lambda)\right)(\wp) \cong \mathbf{D}\left(M_{\kappa}(\lambda)\right) \cong M_{\kappa}(\lambda)^{\vee} .
$$

Recall from Lemma 2.1 that $M_{\mathbf{k}}(\lambda)_{K}$ is a simple $\mathcal{U}_{\mathbf{k}, K}$-module. Therefore we have $\left(\mathbf{D} M_{\mathbf{k}}(\lambda)\right)_{K} \cong M_{\mathbf{k}}(\lambda)_{K}$. So the lemma follows from Lemma 1.5 applied to the highest weight category $\left(\mathcal{A}_{\mathbf{k}}, \Delta_{\mathbf{k}}\right)$ and the set $\left\{\mathbf{D} M_{\mathbf{k}}(\lambda) \mid \lambda \in \mathcal{P}_{n}\right\}$.

2.8. Comparison of the Jantzen filtrations. By Definition 1.6 for any $\lambda \in \mathcal{P}_{n}$ there is a Jantzen filtration of $M_{\kappa}(\lambda)$ associated with the highest weight category $\left(\mathcal{A}_{\mathbf{k}}, \Delta_{\mathbf{k}}\right)$. Lemma 2.11 implies that this Jantzen filtration coincides with the one given in Section 2.3 .

2.9. The $v$-Schur algebra. In this section let $R$ denote an arbitrary integral domain. Let $\mathbf{v}$ be an invertible element in $R$. The Hecke algebra $\mathscr{H}_{\mathbf{v}}$ over $R$ is an $R$-algebra, which is free as a $R$-module with basis $\left\{T_{w} \mid w \in \mathfrak{S}_{0}\right\}$, the multiplication is given by

$$
\begin{array}{cc}
T_{w_{1}} T_{w_{2}}=T_{w_{1} w_{2}}, & \text { if } \\
\left(T_{s_{i}}+1\right)\left(T_{s_{i}}-\mathbf{v}\right)=0, & 1 \leqslant i \leqslant m-1 .
\end{array}
$$

Next, recall that a composition of $n$ is a sequence $\mu=\left(\mu_{1}, \ldots, \mu_{d}\right)$ of positive integers such that $\sum_{i=1}^{d} \mu_{i}=n$. Let $\mathcal{X}_{n}$ be the set of compositions of $n$. For $\mu \in \mathcal{X}_{n}$ let $\mathfrak{S}_{\mu}$ be the subgroup of $\mathfrak{S}_{0}$ generated by $s_{i}$ for all $1 \leqslant i \leqslant d-1$ such that $i \neq \mu_{1}+\cdots+\mu_{j}$ for any $j$. Write

$$
x_{\mu}=\sum_{w \in \mathfrak{S}_{\mu}} T_{w} \quad \text { and } \quad y_{\mu}=\sum_{w \in \mathfrak{S}_{\mu}}(-\mathbf{v})^{-l(w)} T_{w} .
$$


The $v$-Schur algebra $\mathbf{S}_{\mathbf{v}}$ of parameter $\mathbf{v}$ is the endomorphism algebra of the right $\mathscr{H}_{\mathbf{v}}$-module $\bigoplus_{\mu \in \mathcal{X}_{n}} x_{\mu} \mathscr{H}_{\mathbf{v}}$. We will abbreviate

$$
\mathcal{A}_{\mathbf{v}}=\mathbf{S}_{\mathbf{v}}-\bmod \text {. }
$$

Consider the composition $\varpi$ of $n$ such that $\varpi_{i}=1$ for $1 \leqslant i \leqslant n$. Then $x_{\varpi} \mathscr{H}_{\mathbf{v}}=$ $\mathscr{H}_{\mathbf{v}}$. So the Hecke algebra $\mathscr{H}_{\mathbf{v}}$ identifies with a subalgebra of $\mathbf{S}_{\mathbf{v}}$ via the canonical isomorphism $\mathscr{H}_{\mathbf{v}} \cong \operatorname{End} \mathscr{H}_{\mathbf{v}}\left(\mathscr{H}_{\mathbf{v}}\right)$.

For $\lambda \in \mathcal{P}_{n}$ let $\lambda^{\prime}$ be the transposed partition of $\lambda$. Let $\varphi_{\lambda}$ be the element in $\mathbf{S}_{\mathbf{v}}$ given by $\varphi_{\lambda}(h)=x_{\lambda} h$ for $h \in x_{\varpi} \mathscr{H}_{\mathbf{v}}$ and $\varphi_{\lambda}\left(x_{\mu} \mathscr{H}_{\mathbf{v}}\right)=0$ for any composition $\mu \neq \varpi$. Then there is a particular element $w_{\lambda} \in \mathfrak{S}_{0}$ associated with $\lambda$ such that the Weyl module $W_{\mathbf{v}}(\lambda)$ is the left ideal in $\mathbf{S}_{\mathbf{v}}$ generated by the element

$$
z_{\lambda}=\varphi_{\lambda} T_{w_{\lambda}} y_{\lambda^{\prime}} \in \mathbf{S}_{\mathbf{v}}
$$

See [JM] for details. We will write

$$
\Delta_{\mathbf{v}}=\left\{W_{\mathbf{v}}(\lambda) \mid \lambda \in \mathcal{P}_{n}\right\} .
$$

2.10. The Jantzen filtration of Weyl modules. Now, set again $R=\mathbb{C}[[s]]$. Fix

$$
v=\exp (2 \pi i / \kappa) \in \mathbb{C} \quad \text { and } \quad \mathbf{v}=\exp (2 \pi i / \mathbf{k}) \in R .
$$

Below we will consider the $v$-Schur algebra over $\mathbb{C}$ with the parameter $v$, and the $v$-Schur algebra over $R$ with the parameter $\mathbf{v}$. The category $\left(\mathcal{A}_{v}, \Delta_{v}\right)$ is a highest weight $\mathbb{C}$-category. Write $L_{v}(\lambda)$ for the simple quotient of $W_{v}(\lambda)$. The canonical algebra isomorphism $\mathbf{S}_{\mathbf{v}}(\wp) \cong \mathbf{S}_{v}$ implies that $\left(\mathcal{A}_{\mathbf{v}}, \Delta_{\mathbf{v}}\right)$ is a highest weight $R$ category and there is a canonical equivalence

$$
\left(\mathcal{A}_{\mathbf{v}}(\wp), \Delta_{\mathbf{v}}(\wp)\right) \cong\left(\mathcal{A}_{v}, \Delta_{v}\right) .
$$

We define the Jantzen filtration $\left(J^{i} W_{v}(\lambda)\right)$ of $W_{v}(\lambda)$ by applying Definition 1.6 to $\left(\mathcal{A}_{\mathbf{v}}, \Delta_{\mathbf{v}}\right)$. This filtration coincides with the one defined in [JM], because the contravariant form on $W_{\mathbf{v}}(\lambda)$ used in [JM]'s definition is equivalent to a morphism from $W_{\mathbf{v}}(\lambda)$ to the dual standard module $W_{\mathbf{v}}(\lambda)^{\vee}=\operatorname{Hom}_{R}\left(W_{\mathbf{v}}(\lambda), R\right)$.

2.11. Equivalence of $\mathcal{A}_{\mathbf{k}}$ and $\mathcal{A}_{\mathbf{v}}$. In this section we will show that the highest weight $R$-categories $\mathcal{A}_{\mathrm{k}}$ and $\mathcal{A}_{\mathrm{v}}$ are equivalent. The proof uses rational double affine Hecke algebras and Suzuki's functor. Let us first give some reminders. Let $\mathfrak{h}=\mathbb{C}^{n}$, let $y_{1}, \ldots, y_{n}$ be its standard basis and $x_{1}, \ldots, x_{n} \in \mathfrak{h}^{*}$ be the dual basis. Let $\mathbf{H}_{1 / \kappa}$ be the rational double affine Hecke algebra associated with $\mathfrak{S}_{n}$ with parameter $1 / \kappa$. It is the quotient of the smash product of the tensor algebra $T\left(\mathfrak{h} \oplus \mathfrak{h}^{*}\right)$ with $\mathbb{C S}_{n}$ by the relations

$$
\left[y_{i}, x_{i}\right]=1+\frac{1}{\kappa} \sum_{j \neq i} s_{i j}, \quad\left[y_{i}, x_{j}\right]=\frac{-1}{\kappa} s_{i j}, \quad 1 \leqslant i, j \leqslant n, i \neq j .
$$

Here $s_{i j}$ denotes the element of $\mathfrak{S}_{n}$ that permutes $i$ and $j$. Denote by $\mathcal{B}_{\kappa}$ the category $\mathcal{O}$ of $\mathbf{H}_{1 / \kappa}$ as defined in GGOR. It is a highest weight $\mathbb{C}$-category. Let $\left\{B_{\kappa}(\lambda) \mid \lambda \in \mathcal{P}_{n}\right\}$ be the set of standard modules.

Now, let $V=\mathbb{C}^{m}$ be the dual of the vectorial representation of $\mathfrak{g}_{0}$. For any object $M$ in $\mathcal{A}_{\kappa}$ consider the action of the Lie algebra $\mathfrak{g}_{0} \otimes \mathbb{C}[z]$ on the vector space

$$
T(M)=V^{\otimes n} \otimes M \otimes \mathbb{C}[\mathfrak{h}]
$$


given by

$$
\left(\xi \otimes z^{a}\right)(v \otimes m \otimes f)=\sum_{i=1}^{n} \xi_{(i)}(v) \otimes m \otimes x_{i}^{a} f+v \otimes(-1)^{a}\left(\xi \otimes t^{-a}\right) m \otimes f
$$

for $\xi \in \mathfrak{g}_{0}, a \in \mathbb{N}, v \in V^{\otimes n}, m \in M, f \in \mathbb{C}[\mathfrak{h}]$. Here $\xi_{(i)}$ is the operator on $V^{\otimes n}$ that acts on the $i$-th copy of $V$ by $\xi$ and acts on the other copies of $V$ by identity. Suzuki defined a natural action of $\mathbf{H}_{1 / \kappa}$ on the space of coinvariants

$$
\mathfrak{E}_{\kappa}(M)=H_{0}\left(\mathfrak{g}_{0} \otimes \mathbb{C}[z], T(M)\right) .
$$

The assignment $M \mapsto \mathfrak{E}_{\kappa}(M)$ gives a right exact functor

$$
\mathfrak{E}_{\kappa}: \mathcal{A}_{\kappa} \rightarrow \mathcal{B}_{\kappa}
$$

See $[\mathrm{Su}$ or VV2, Section 2] for details. We have

$$
\mathfrak{E}_{\kappa}\left(M_{\kappa}(\lambda)\right)=B_{\kappa}(\lambda),
$$

and $\mathfrak{E}_{\kappa}$ is an equivalence of highest weight categories [VV2, Theorem A.5.1].

Next, we consider the rational double affine Hecke algebra $\mathbf{H}_{1 / \mathbf{k}}$ over $R$ with parameter $1 / \mathbf{k}$. The category $\mathcal{O}$ of $\mathbf{H}_{1 / \mathbf{k}}$ is defined in the obvious way. It is a highest weight $R$-category. We will denote it by $\mathcal{B}_{\mathbf{k}}$. The standard modules will be denoted by $B_{\mathbf{k}}(\lambda)$. The Suzuki functor over $R$,

$$
\mathfrak{E}_{\mathbf{k}}: \mathcal{A}_{\mathbf{k}} \rightarrow \mathcal{B}_{\mathbf{k}}, \quad M \mapsto H_{0}\left(\mathfrak{g}_{0} \otimes \mathbb{C}[z], T(M)\right) .
$$

is defined in the same way. It has the following properties.

Lemma 2.12. (a) We have $\mathfrak{E}_{\mathbf{k}}\left(M_{\mathbf{k}}(\lambda)\right)=B_{\mathbf{k}}(\lambda)$ for $\lambda \in \mathcal{P}_{n}$

(b) The functor $\mathfrak{E}_{\mathbf{k}}$ restricts to an exact functor $\mathcal{A}_{\mathbf{k}}^{\Delta} \rightarrow \mathcal{B}_{\mathbf{k}}^{\Delta}$.

(c) The functor $\mathfrak{E}_{\mathbf{k}}$ maps a projective generator of $\mathcal{A}_{\mathbf{k}}$ to a projective generator of $\mathcal{B}_{\mathbf{k}}$.

Proof. The proof of part (a) is the same as in the nondeformed case. For part (b), since $\mathfrak{E}_{\mathbf{k}}$ is right exact over $\mathcal{A}_{\mathbf{k}}$, it is enough to prove that for any injective morphism $f: M \rightarrow N$ with $M, N \in \mathcal{A}_{\mathbf{k}}^{\Delta}$ the map

$$
\mathfrak{E}_{\mathbf{k}}(f): \mathfrak{E}_{\mathbf{k}}(M) \rightarrow \mathfrak{E}_{\mathbf{k}}(N)
$$

is injective. Recall from Lemma 2.1 that the $\mathcal{U}_{\mathbf{k}, K}$-module $M_{\mathbf{k}}(\lambda)_{K}$ is simple for any $\lambda$. So the functor

$$
\mathfrak{E}_{\mathbf{k}, K}: \mathcal{A}_{\mathbf{k}, K} \rightarrow \mathcal{B}_{\mathbf{k}, K}
$$

is an equivalence. Hence the map

$$
\mathfrak{E}_{\mathbf{k}}(f) \otimes_{R} K: \mathfrak{E}_{\mathbf{k}, K}\left(M_{K}\right) \rightarrow \mathfrak{E}_{\mathbf{k}, K}\left(N_{K}\right)
$$

is injective. Since both $\mathfrak{E}_{\mathbf{k}}(M)$ and $\mathfrak{E}_{\mathbf{k}}(N)$ are free $R$-modules, this implies that $\mathfrak{E}_{\mathbf{k}}(f)$ is also injective. Now, let us concentrate on (c). Let $P$ be a projective generator of $\mathcal{A}_{\mathbf{k}}$. Then $P(\wp)$ is a projective generator of $\mathcal{A}_{\kappa}$. Since $\mathfrak{E}_{\kappa}$ is an equivalence of categories, we have $\mathfrak{E}_{\kappa}(P(\wp))$ is a projective generator of $\mathcal{B}_{\kappa}$. By (b) the object $\mathfrak{E}_{\mathbf{k}}(P)$ belongs to $\mathcal{B}_{\mathbf{k}}^{\Delta}$, so it is free over $R$. Therefore by the Universal Coefficient Theorem we have

$$
\left(\mathfrak{E}_{\mathbf{k}}(P)\right)(\wp) \cong \mathfrak{E}_{\kappa}(P(\wp)) .
$$

Hence $\mathfrak{E}_{\mathbf{k}}(P)$ is a projective object of $\mathcal{B}_{\mathbf{k}}$. Note that for any $\lambda \in \mathcal{P}_{n}$ there is a surjective map $P \rightarrow M_{\mathbf{k}}(\lambda)$. The right exact functor $\mathfrak{E}_{\mathbf{k}}$ sends it to a surjective map $\mathfrak{E}_{\mathbf{k}}(P) \rightarrow B_{\mathbf{k}}(\lambda)$. This proves that $\mathfrak{E}_{\mathbf{k}}(P)$ is a projective generator of $\mathcal{B}_{\mathbf{k}}$. 
Proposition 2.13. Assume that $\kappa \leqslant-3$. Then there exists an equivalence of highest weight $R$-categories

$$
\mathcal{A}_{\mathbf{k}} \stackrel{\sim}{\rightarrow} \mathcal{A}_{\mathbf{v}}
$$

which maps $M_{\mathbf{k}}(\lambda)$ to $W_{\mathbf{v}}(\lambda)$ for any $\lambda \in \mathcal{P}_{n}$.

Proof. We first give an equivalence of highest weight categories

$$
\Phi: \mathcal{A}_{\mathrm{k}} \rightarrow \mathcal{B}_{\mathrm{k}}
$$

as follows. Let $P$ be a projective generator of $\mathcal{A}_{\mathbf{k}}$. Then $Q=\mathfrak{E}_{\mathbf{k}}(P)$ is a projective generator of $\mathcal{B}_{\mathrm{k}}$ by Lemma 2.12(c). By Morita theory we have equivalences of categories

$$
\begin{aligned}
\operatorname{Hom}_{\mathcal{A}_{\mathbf{k}}}(P,-): \mathcal{A}_{\mathbf{k}} \stackrel{\sim}{\rightarrow} \operatorname{End}_{\mathcal{A}_{\mathbf{k}}}(P)^{\mathrm{op}}-\bmod , \\
\operatorname{Hom}_{\mathcal{B}_{\mathbf{k}}}(Q,-): \mathcal{B}_{\mathbf{k}} \stackrel{\sim}{\rightarrow} \operatorname{End}_{\mathcal{B}_{\mathbf{k}}}(Q)^{\mathrm{op}}-\bmod .
\end{aligned}
$$

We claim that the algebra homomorphism

$$
\operatorname{End}_{\mathcal{A}_{\mathbf{k}}}(P) \rightarrow \operatorname{End}_{\mathcal{B}_{\mathbf{k}}}(Q), \quad f \mapsto \mathfrak{E}_{\mathbf{k}}(f),
$$

is an isomorphism. To see this, note that we have

$$
\begin{gathered}
Q(\wp)=\mathfrak{E}_{\kappa}(P(\wp)), \quad\left(\operatorname{End}_{\mathcal{A}_{\mathbf{k}}}(P)\right)(\wp)=\operatorname{End}_{\mathcal{A}_{\kappa}}(P(\wp)), \\
\left(\operatorname{End}_{\mathcal{B}_{\mathbf{k}}}(Q)\right)(\wp)=\operatorname{End}_{\mathcal{B}_{\kappa}}(Q(\wp)) .
\end{gathered}
$$

Since $\mathfrak{E}_{\kappa}$ is an equivalence, it yields an isomorphism

$$
\operatorname{End}_{\mathcal{A}_{\kappa}}(P(\wp)) \stackrel{\sim}{\rightarrow} \operatorname{End}_{\mathcal{B}_{\kappa}}(Q(\wp)), \quad f \mapsto \mathfrak{E}_{\kappa}(f) .
$$

Since both $\operatorname{End}_{\mathcal{A}_{\mathbf{k}}}(P)$ and $\operatorname{End}_{\mathcal{B}_{\mathbf{k}}}(Q)$ are finitely generated free $R$-modules, by Nakayama's lemma the morphism (2.10) is an isomorphism. In particular, it yields an equivalence of categories

$$
\operatorname{End}_{\mathcal{A}_{\mathbf{k}}}(P)^{\mathrm{op}}-\bmod \cong \operatorname{End}_{\mathcal{B}_{\mathbf{k}}}(Q)^{\mathrm{op}}-\bmod .
$$

Combined with the other two equivalences above, we get an equivalence of categories

$$
\Phi: \mathcal{A}_{\mathbf{k}} \rightarrow \mathcal{B}_{\mathbf{k}}
$$

It remains to show that

$$
\Phi\left(M_{\mathbf{k}}(\lambda)\right) \cong B_{\mathbf{k}}(\lambda), \quad \lambda \in \mathcal{P}_{n} .
$$

Note that the functor $\mathfrak{E}_{\mathrm{k}}$ yields a morphism of finitely generated $R$-modules

$$
\begin{aligned}
\operatorname{Hom}_{\mathcal{B}_{\mathbf{k}}}\left(Q, \Phi\left(M_{\mathbf{k}}(\lambda)\right)\right) & =\operatorname{End}_{\mathcal{B}_{\mathbf{k}}}(Q)^{\mathrm{op}} \otimes_{\operatorname{End}_{\mathcal{A}_{\mathbf{k}}}(P)^{\mathrm{op}}} \operatorname{Hom}_{\mathcal{A}_{\mathbf{k}}}\left(P, M_{\mathbf{k}}(\lambda)\right) \\
& \rightarrow \operatorname{Hom}_{\mathcal{B}_{\mathbf{k}}}\left(Q, \mathfrak{E}_{\mathbf{k}}\left(M_{\mathbf{k}}(\lambda)\right)\right) \\
& =\operatorname{Hom}_{\mathcal{B}_{\mathbf{k}}}\left(Q, B_{\mathbf{k}}(\lambda)\right)
\end{aligned}
$$

Let us denote it by $\varphi$. Note also that we have isomorphisms

$$
\begin{aligned}
\operatorname{Hom}_{\mathcal{A}_{\mathbf{k}}}\left(P, M_{\mathbf{k}}(\lambda)\right)(\wp) & =\operatorname{Hom}_{\mathcal{A}_{\kappa}}\left(P(\wp), M_{\kappa}(\lambda)\right), \\
\operatorname{Hom}_{\mathcal{B}_{\mathbf{k}}}\left(Q, B_{\mathbf{k}}(\lambda)\right)(\wp) & =\operatorname{Hom}_{\mathcal{B}_{\kappa}}\left(Q(\wp), B_{\kappa}(\lambda)\right),
\end{aligned}
$$

and note that $\mathfrak{E}_{\kappa}$ is an equivalence of categories. So the map $\varphi(\wp)$ is an isomorphism. Furthermore, $\operatorname{Hom}_{\mathcal{B}_{\mathbf{k}}}\left(Q, B_{\mathbf{k}}(\lambda)\right)$ is free over $R$, so Nakayama's lemma implies that $\varphi$ is also an isomorphism. The preimage of $\varphi$ under the equivalence $\operatorname{Hom}_{\mathcal{B}_{\mathbf{k}}}(Q,-)$ yields an isomorphism

$$
\Phi\left(M_{\mathbf{k}}(\lambda)\right) \simeq B_{\mathbf{k}}(\lambda)
$$


Finally, if $v \neq-1$, i.e., $\kappa \leqslant-3$, then by $\left[\mathrm{R}\right.$, Theorem 6.8] the categories $\mathcal{B}_{\mathbf{k}}$ and $\mathcal{A}_{\mathbf{v}}$ are equivalent highest weight $R$-categories with $B_{\mathbf{k}}(\lambda)$ corresponding to $W_{\mathbf{v}}(\lambda)$. This equivalence composed with $\Phi$ gives the desired equivalence in the proposition.

Corollary 2.14. Assume that $\kappa \leqslant-3$. Then for any $\lambda, \mu \in \mathcal{P}_{n}$ and $i \in \mathbb{N}$ we have

$$
\left[J^{i} M_{\kappa}(\lambda) / J^{i+1} M_{\kappa}(\lambda): L_{\kappa}(\mu)\right]=\left[J^{i} W_{v}(\lambda) / J^{i+1} W_{v}(\lambda): L_{v}(\mu)\right] .
$$

Proof. This follows from the proposition above and Proposition 1.8 .

To prove the main theorem, it remains to compute the left hand side of (2.11). This will be done by generalizing the approach of $[\mathrm{BB}]$ to the affine parabolic case. To this end, we first give some reminders on $\mathscr{D}$-modules on affine flag varieties.

\section{Generalities on $\mathscr{D}$-MOdules on ind-Schemes}

In this section, we first recall basic notion for $\mathscr{D}$-modules on (possibly singular) schemes. We will also discuss twisted $\mathscr{D}$-modules and holonomic $\mathscr{D}$-modules. Then we introduce the notion of $\mathscr{D}$-modules on ind-schemes following $[\mathrm{BD}]$ and $[\mathrm{KV}]$.

3.1. Reminders on $\mathscr{D}$-modules. Unless specified otherwise, all the schemes will be assumed to be of finite type over $\mathbb{C}$, quasi-separated and quasi-projective. Although a large number of statements are true in a larger generality, we will only use them for quasi-projective schemes. For any scheme $Z$, let $\mathscr{O}_{Z}$ be the structure sheaf over $Z$. We write $\mathbf{O}(Z)$ for the category of quasi-coherent $\mathscr{O}_{Z}$-modules on $Z$. Note that we abbreviate $\mathscr{O}_{Z}$-module for sheaf of $\mathscr{O}_{Z}$-modules over $Z$. For $f: Z \rightarrow Y$ a morphism of schemes, we write $f_{*}, f^{*}$ for the functors of direct and inverse images on $\mathbf{O}(Z), \mathbf{O}(Y)$. If $f$ is a closed embedding and $\mathscr{M} \in \mathbf{O}(Y)$, we consider the quasi-coherent $\mathscr{O}_{Z}$-module

$$
f^{!} \mathscr{M}=f^{-1} \mathscr{H}_{o m_{O_{Y}}}\left(f_{*} \mathscr{O}_{Z}, \mathscr{M}\right) .
$$

It is the restriction to $Z$ of the subsheaf of $\mathscr{M}$ consisting of sections supported scheme-theoretically on $f(Z) \subset Y$.

Let $Z$ be a smooth scheme. Let $\mathscr{D}_{Z}$ be the ring of differential operators on $Z$. We denote by $\mathbf{M}(Z)$ the category of right $\mathscr{D}_{Z}$-modules that are quasi-coherent as $\mathscr{O}_{Z}$-modules. It is an abelian category. Let $\Omega_{Z}$ denote the sheaf of differential forms of highest degree on $Z$. The category of right $\mathscr{D}_{Z}$-modules is equivalent to the category of left $\mathscr{D}_{Z}$-modules via $\mathscr{M} \mapsto \Omega_{Z} \otimes_{\mathscr{O}_{Z}} \mathscr{M}$. Let $i: Y \rightarrow Z$ be a morphism of smooth schemes. We consider the $\left(\mathscr{D}_{Y}, i^{-1} \mathscr{D}_{Z}\right)$-bimodule

$$
\mathscr{D}_{Y \rightarrow Z}=i^{*} \mathscr{D}_{Z}=\mathscr{O}_{Y} \otimes_{i^{-1} \mathscr{O}_{Z}} i^{-1} \mathscr{D}_{Z} \text {. }
$$

We define the following functors:

$$
\begin{aligned}
i^{*}: \mathbf{M}(Z) & \rightarrow \mathbf{M}(Y), & \mathscr{M} & \mapsto \Omega_{Y} \otimes_{\mathscr{O}_{Y}}\left(\mathscr{D}_{Y \rightarrow Z} \otimes_{\mathscr{D}_{Z}}\left(\Omega_{Z} \otimes_{\mathscr{O}_{Z}} \mathscr{M}\right)\right), \\
i_{\bullet}: \mathbf{M}(Y) & \rightarrow \mathbf{M}(Z), & \mathscr{M} & \mapsto i_{*}\left(\mathscr{M} \otimes_{\mathscr{D}_{Y}} \mathscr{D}_{Y \rightarrow Z}\right) .
\end{aligned}
$$

For any $\mathscr{M} \in \mathbf{M}(Y)$ let $\mathscr{M}^{\mathscr{O}}$ denote the underlying $\mathscr{O}_{Y}$-module of $\mathscr{M}$. Then we have

$$
i^{*}\left(\mathscr{M}^{\mathscr{O}}\right)=i^{*}(\mathscr{M})^{\mathscr{O}}
$$

If $i$ is a locally closed affine embedding, then the functor $i_{\bullet}$ is exact. For any closed subscheme $Z^{\prime}$ of $Z$, we denote by $\mathbf{M}\left(Z, Z^{\prime}\right)$ the full subcategory of $\mathbf{M}(Z)$ consisting of $\mathscr{D}_{Z}$-modules supported set-theoretically on $Z^{\prime}$. If $i: Y \rightarrow Z$ is a 
closed embedding of smooth varieties, then by a theorem of Kashiwara, the functor $i$. yields an equivalence of categories

$$
\mathbf{M}(Y) \cong \mathbf{M}(Z, Y) .
$$

We refer to [HTT for more details about $\mathscr{D}$-modules on smooth schemes

Now, let $Z$ be a possibly singular scheme. We consider the abelian category $\mathbf{M}(Z)$ of right $\mathscr{D}$-modules on $Z$ with a faithful forgetful functor

$$
\mathbf{M}(Z) \rightarrow \mathbf{O}(Z), \quad \mathscr{M} \mapsto \mathscr{M}^{\mathscr{O}}
$$

as in [BD, 7.10.3]. If $Z$ is smooth, it is equivalent to the category $\mathbf{M}(Z)$ above; see [BD, 7.10.12]. For any closed embedding $i: Z \rightarrow X$ there is a left exact functor

$$
i^{!}: \mathbf{M}(X) \rightarrow \mathbf{M}(Z)
$$

such that $\left(i^{!}(\mathscr{M})\right)^{\mathscr{O}}=i^{!}\left(\mathscr{M}^{\mathscr{O}}\right)$ for all $\mathscr{M}$. It admits an exact left adjoint functor

$$
i_{\bullet}: \mathbf{M}(Z) \rightarrow \mathbf{M}(X) .
$$

In the smooth case these functors coincide with the one before. If $X$ is smooth, then $i$. and $i^{!}$yield mutually inverse equivalences of categories

$$
\mathbf{M}(Z) \cong \mathbf{M}(X, Z)
$$

such that $i^{!} \circ i_{\bullet}=\mathrm{Id}$; see $\left.\mathrm{BD}, 7.10 .11\right]$. Note that when $Z$ is smooth, this is Kashiwara's equivalence (3.1). We will always consider $\mathscr{D}$-modules on a (possibly singular) scheme $Z$ which is given an embedding into a smooth scheme. Finally, if $j: Y \rightarrow Z$ is a locally closed affine embedding and $Y$ is smooth, then we have the following exact functor

$$
j_{\bullet}=i^{!} \circ(i \circ j) \bullet: \mathbf{M}(Y) \rightarrow \mathbf{M}(Z) .
$$

Its definition is independent of the choice of $i$.

3.2. Holonomic $\mathscr{D}$-modules. Let $Z$ be a scheme. If $Z$ is smooth, we denote by $\mathbf{M}_{h}(Z)$ the category of holonomic $\mathscr{D}_{Z}$-modules; see e.g., [HTT, Definition 2.3.6]. Otherwise, let $i: Z \rightarrow X$ be a closed embedding into a smooth scheme $X$. We define $\mathbf{M}_{h}(Z)$ to be the full subcategory of $\mathbf{M}(Z)$ consisting of objects $\mathscr{M}$ such that $i \cdot \mathscr{M}$ is holonomic. The category $\mathbf{M}_{h}(Z)$ is abelian. There is a (contravariant) duality functor on $\mathbf{M}_{h}(Z)$ given by

$$
\mathbb{D}: \mathbf{M}_{h}(Z) \rightarrow \mathbf{M}_{h}(Z), \quad \mathscr{M} \mapsto i^{!}\left(\Omega_{X} \otimes_{\mathscr{O}_{X}} \mathscr{E}_{x} t_{\mathscr{D}_{X}}^{\operatorname{dim} X}\left(i_{\bullet} \mathscr{M}, \mathscr{D}_{X}\right)\right) .
$$

For a locally closed affine embedding $i: Y \rightarrow Z$ with $Y$ a smooth scheme, the functor $i \bullet$ given by (3.3) maps $\mathbf{M}_{h}(Y)$ to $\mathbf{M}_{h}(Z)$. We put

$$
i_{!}=\mathbb{D} \circ i_{\bullet} \circ \mathbb{D}: \mathbf{M}_{h}(Y) \rightarrow \mathbf{M}_{h}(Z) .
$$

There is a canonical morphism of functors

$$
\psi: i_{!} \rightarrow i_{\bullet}
$$

The intermediate extension functor is given by

$$
i_{!}: \mathbf{M}_{h}(Y) \rightarrow \mathbf{M}_{h}(Z), \quad \mathscr{M} \mapsto \operatorname{Im}\left(\psi(\mathscr{M}): i_{!} \mathscr{M} \rightarrow i_{\bullet} \mathscr{M}\right) .
$$

Note that the functors $i_{\bullet}, i_{\text {! }}$ are exact. Moreover, if the embedding $i$ is closed, then $\psi$ is an isomorphism of functors $i_{!} \cong i_{\bullet}$. 
3.3. Weakly equivariant $\mathscr{D}$-modules. Let $T$ be a linear group. For any $T$ scheme $Z$ there is an abelian category $\mathbf{M}^{T}(Z)$ of weakly $T$-equivariant right $\mathscr{D}$ modules on $Z$ with a faithful forgetful functor

$$
\mathbf{M}^{T}(Z) \rightarrow \mathbf{M}(Z)
$$

If $Z$ is smooth, an object $\mathscr{M}$ of $\mathbf{M}^{T}(Z)$ is an object $\mathscr{M}$ of $\mathbf{M}(Z)$ equipped with a structure of $T$-equivariant $\mathscr{O}_{Z}$-module such that the action map $\mathscr{M}_{\mathscr{O}_{Z}} \mathscr{D}_{Z} \rightarrow \mathscr{M}$ is $T$-equivariant. For any $T$-scheme $Z$ with a $T$-equivariant closed embedding $i$ : $Z \rightarrow X$ into a smooth $T$-scheme $X$, the functor $i$. yields an equivalence $\mathbf{M}^{T}(Z) \cong$ $\mathbf{M}^{T}(X, Z)$, where $\mathbf{M}^{T}(X, Z)$ is the subcategory of $\mathbf{M}^{T}(X)$ consisting of objects supported set-theoretically on $Z$.

3.4. Twisted $\mathscr{D}$-modules. Let $T$ be a torus, and let $\mathfrak{t}$ be its Lie algebra. Let $\pi: Z^{\dagger} \rightarrow Z$ be a right $T$-torsor over the scheme $Z$. For any object $\mathscr{M} \in \mathbf{M}^{T}\left(Z^{\dagger}\right)$ the $\mathscr{O}_{Z}$-module $\pi_{*}\left(\mathscr{M}^{\mathscr{O}}\right)$ carries a $T$-action. Let

$$
\mathscr{M}^{\dagger}=\pi_{*}\left(\mathscr{M}^{\mathscr{O}}\right)^{T}
$$

be the $\mathscr{O}_{Z}$-submodule of $\pi_{*}\left(\mathscr{M}^{\mathscr{O}}\right)$ consisting of the $T$-invariant local sections. We have

$$
\Gamma\left(Z, \mathscr{M}^{\dagger}\right)=\Gamma\left(Z^{\dagger}, \mathscr{M}\right)^{T} .
$$

For any weight $\lambda \in \mathfrak{t}^{*}$ we define the categories $\mathbf{M}^{\tilde{\lambda}}(Z), \mathbf{M}^{\lambda}(Z)$ as follows.

First, assume that $Z$ is a smooth scheme. Then $Z^{\dagger}$ is also smooth. So we have a sheaf of algebras on $Z$ given by

$$
\mathscr{D}_{Z}^{\dagger}=\left(\mathscr{D}_{Z^{\dagger}}\right)^{\dagger},
$$

and $\mathscr{M}^{\dagger}$ is a right $\mathscr{D}_{Z}^{\dagger}$-module for any $\mathscr{M} \in \mathbf{M}^{T}\left(Z^{\dagger}\right)$. For any open subscheme $U \subset Z$ the $T$-action on $\pi^{-1}(U)$ yields an algebra homomorphism

$$
\delta_{r}: \mathcal{U}(\mathfrak{t}) \rightarrow \Gamma\left(U, \mathscr{D}_{Z}^{\dagger}\right),
$$

whose image lies in the center of the right hand side. Thus there is also an action of $\mathcal{U}(\mathfrak{t})$ on $\mathscr{M}^{\dagger}$ commuting with the $\mathscr{D}_{Z}^{\dagger}$-action. For $\lambda \in \mathfrak{t}^{*}$ let $\mathfrak{m}_{\lambda} \subset \mathcal{U}(\mathfrak{t})$ be the ideal generated by

$$
\{h+\lambda(h) \mid h \in \mathfrak{t}\} .
$$

We define $\mathbf{M}^{\lambda}(Z)$ (resp. $\mathbf{M}^{\tilde{\lambda}}(Z)$ ) to be the full subcategory of $\mathbf{M}^{T}\left(Z^{\dagger}\right)$ consisting of the objects $\mathscr{M}$ such that the action of $\mathfrak{m}_{\lambda}$ on $\mathscr{M}^{\dagger}$ is zero (resp. nilpotent). In particular $\mathbf{M}^{\lambda}(Z)$ is a full subcategory of $\mathbf{M}^{\tilde{\lambda}}(Z)$ and both categories are abelian. We will write

$$
\Gamma(Z, \mathscr{M})=\Gamma\left(Z, \mathscr{M}^{\dagger}\right), \quad \forall \mathscr{M} \in \mathbf{M}^{\tilde{\lambda}}(Z) .
$$

Now, let $Z$ be any scheme. We say that a $T$-torsor $\pi: Z^{\dagger} \rightarrow Z$ is admissible if there exists a $T$-torsor $X^{\dagger} \rightarrow X$ with $X$ smooth and a closed embedding $i: Z \rightarrow X$ such that $Z^{\dagger} \cong X^{\dagger} \times_{X} Z$ as a $T$-scheme over $Z$. We will only use admissible $T$-torsors. Let $\mathbf{M}^{\lambda}(X, Z), \mathbf{M}^{\tilde{\lambda}}(X, Z)$ be respectively the subcategories of $\mathbf{M}^{\lambda}(X)$, $\mathbf{M}^{\tilde{\lambda}}(X)$ consisting of objects supported on $Z^{\dagger}$. We define $\mathbf{M}^{\lambda}(Z), \mathbf{M}^{\tilde{\lambda}}(Z)$ to be the full subcategories of $\mathbf{M}^{T}\left(Z^{\dagger}\right)$ consisting of objects $\mathscr{M}$ such that $i_{\bullet}(\mathscr{M})$ belongs to $\mathbf{M}^{\lambda}(X, Z), \mathbf{M}^{\tilde{\lambda}}(X, Z)$ respectively. Their definition only depends on $\pi$. 
Remark 3.1. Let $Z$ be a smooth scheme. Let $\mathbf{M}\left(\mathscr{D}_{Z}^{\dagger}\right)$ be the category of right $\mathscr{D}_{Z}^{\dagger}$-modules on $Z$ that are quasi-coherent as $\mathscr{O}_{Z}$-modules. The functor

$$
\mathbf{M}^{T}\left(Z^{\dagger}\right) \stackrel{\sim}{\rightarrow} \mathbf{M}\left(\mathscr{D}_{Z}^{\dagger}\right), \quad \mathscr{M} \mapsto \mathscr{M}^{\dagger}
$$

is an equivalence of categories. A quasi-inverse is given by $\pi^{*}$; see e.g., [BB, Lemma 1.8.10].

Remark 3.2. We record the following fact for a further use. For any smooth $T$-torsor $\pi: Z^{\dagger} \rightarrow Z$, the exact sequence of relative differential 1-forms

$$
\pi^{*}\left(\Omega_{Z}^{1}\right) \longrightarrow \Omega_{Z^{\dagger}}^{1} \longrightarrow \Omega_{Z^{\dagger} / Z}^{1} \longrightarrow 0
$$

yields an isomorphism

$$
\Omega_{Z^{\dagger}}=\pi^{*}\left(\Omega_{Z}\right) \otimes_{\mathscr{O}_{Z^{\dagger}}} \Omega_{Z^{\dagger} / Z}
$$

Since $\pi$ is a $T$-torsor we have indeed

$$
\Omega_{Z^{\dagger} / Z}=\mathscr{O}_{Z^{\dagger}}
$$

as a line bundle. Therefore we have an isomorphism of $\mathscr{O}_{Z^{\dagger}}$-modules

$$
\Omega_{Z^{\dagger}}=\pi^{*}\left(\Omega_{Z}\right) \text {. }
$$

Below, we will identify them whenever needed.

3.5. Twisted holonomic $\mathscr{D}$-modules and duality functors. Let $\pi: Z^{\dagger} \rightarrow Z$ be an admissible $T$-torsor. We define $\mathbf{M}_{h}^{T}\left(Z^{\dagger}\right)$ to be the full subcategory of $\mathbf{M}^{T}\left(Z^{\dagger}\right)$ consisting of objects $\mathscr{M}$ whose image via the functor (3.4) belongs to $\mathbf{M}_{h}\left(Z^{\dagger}\right)$. We define the categories $\mathbf{M}_{h}^{\lambda}(Z), \mathbf{M}_{h}^{\tilde{\lambda}}(Z)$ in the same manner.

Assume that $Z$ is smooth. Then the category $\mathbf{M}^{T}\left(Z^{\dagger}\right)$ has enough injective objects; see e.g., [Kas, Proposition 3.3.5] and the references there. We define a (contravariant) duality functor on $\mathbf{M}_{h}^{T}\left(Z^{\dagger}\right)$ by

$$
\mathbb{D}^{\prime}: \mathbf{M}_{h}^{T}\left(Z^{\dagger}\right) \rightarrow \mathbf{M}_{h}^{T}\left(Z^{\dagger}\right), \quad \mathscr{M} \mapsto \Omega_{Z^{\dagger}} \otimes_{\mathscr{O}_{Z^{\dagger}}} \mathscr{E} x t_{\mathbf{M}^{T}\left(Z^{\dagger}\right)}^{\operatorname{dim} Z^{\dagger}}\left(\mathscr{M}, \mathscr{D}_{Z^{\dagger}}\right) .
$$

We may write $\mathbb{D}^{\prime}=\mathbb{D}_{Z}^{\prime}$. Note that by Remark 3.2 and the equivalence (3.7) we have

$$
\left(\mathbb{D}_{Z}^{\prime} \mathscr{M}\right)^{\dagger}=\Omega_{Z} \otimes_{\mathscr{O}_{Z}} \mathscr{E} x t_{\mathscr{D}_{Z}^{\dagger}}^{\operatorname{dim} Z^{\dagger}}\left(\mathscr{M}^{\dagger}, \mathscr{D}_{Z}^{\dagger}\right), \quad \forall \mathscr{M} \in \mathbf{M}^{T}\left(Z^{\dagger}\right) .
$$

For any $\lambda \in \mathfrak{t}^{*}$ the functor $\mathbb{D}^{\prime}$ restricts to (contravariant) equivalences of categories

$$
\mathbb{D}^{\prime}: \mathbf{M}_{h}^{\tilde{\lambda}}(Z) \rightarrow \mathbf{M}_{h}^{\widetilde{-\lambda}}(Z), \quad \mathbb{D}^{\prime}: \mathbf{M}_{h}^{\lambda}(Z) \rightarrow \mathbf{M}_{h}^{-\lambda}(Z)
$$

see e.g., $\mathrm{BB}$, Remark 2.5.5(iv)]. In particular, if $\lambda=0$, then $\mathbb{D}^{\prime}$ yields a duality on $\mathbf{M}_{h}^{0}\left(Z^{\dagger}\right)$. It is compatible with the duality functor $\mathbb{D}$ on $\mathbf{M}_{h}(Z)$ defined in Section 3.2 via the equivalence

$$
\Phi: \mathbf{M}_{h}^{0}(Z) \rightarrow \mathbf{M}_{h}(Z), \quad \mathscr{M} \mapsto \mathscr{M}^{\dagger}
$$

given by (3.7). More precisely, we have the following lemma.

Lemma 3.3. We have $\Phi \circ \mathbb{D}^{\prime}=\mathbb{D} \circ \Phi$.

The proof is standard, and is left to the reader.

Similarly, for an admissible $T$-torsor $\pi: Z^{\dagger} \rightarrow Z$ with an embedding $i$ into a smooth $T$-torsor $X^{\dagger} \rightarrow X$, we define the functor

$$
\mathbb{D}^{\prime}: \mathbf{M}_{h}^{T}\left(Z^{\dagger}\right) \rightarrow \mathbf{M}_{h}^{T}\left(Z^{\dagger}\right), \quad \mathscr{M} \mapsto i^{!} \mathbb{D}_{X}^{\prime}\left(i_{\bullet}(\mathscr{M})\right) .
$$

Its definition only depends on $\pi$. The equivalence (3.9) and Lemma 3.3 hold again. 
A weight $\lambda \in \mathfrak{t}^{*}$ is integral if it is given by the differential of a character $e^{\lambda}$ : $T \rightarrow \mathbb{C}^{*}$. For such a $\lambda$ we consider the invertible sheaf $\mathscr{L}_{Z}^{\lambda} \in \mathbf{O}(Z)$ defined by

$$
\Gamma\left(U, \mathscr{L}_{Z}^{\lambda}\right)=\left\{\gamma \in \Gamma\left(\pi^{-1}(U), \mathscr{O}_{Z^{\dagger}}\right) \mid \gamma\left(x h^{-1}\right)=e^{\lambda}(h) \gamma(x), \quad(x, h) \in \pi^{-1}(U) \times T\right\}
$$

for any open set $U \subset Z$. We define the following translation functor:

$$
\Theta^{\lambda}: \mathbf{M}_{h}^{T}\left(Z^{\dagger}\right) \rightarrow \mathbf{M}_{h}^{T}\left(Z^{\dagger}\right), \quad \mathscr{M} \mapsto \mathscr{M} \otimes_{\mathscr{O}_{Z^{\dagger}}} \pi^{*}\left(\mathscr{L}_{Z}^{\lambda}\right) .
$$

It is an equivalence of categories. A quasi-inverse is given by $\Theta^{-\lambda}$. For any $\mu \in \mathfrak{t}^{*}$ the restriction of $\Theta^{\lambda}$ yields equivalences of categories

$$
\Theta^{\lambda}: \mathbf{M}_{h}^{\tilde{\mu}}(Z) \rightarrow \widetilde{\mathbf{M}_{h}^{\mu+\lambda}}(Z), \quad \Theta^{\lambda}: \mathbf{M}_{h}^{\mu}(Z) \rightarrow \mathbf{M}_{h}^{\mu+\lambda}(Z) .
$$

We define the duality functor on $\mathbf{M}_{h}^{\tilde{\lambda}}(Z)$ to be

$$
\mathbb{D}: \mathbf{M}_{h}^{\tilde{\lambda}}(Z) \rightarrow \mathbf{M}_{h}^{\tilde{\lambda}}(Z), \quad \mathscr{M} \mapsto \Theta^{2 \lambda} \circ \mathbb{D}^{\prime}(\mathscr{M}) .
$$

It restricts to a duality functor on $\mathbf{M}_{h}^{\lambda}(Z)$, which we denote again by $\mathbb{D}$. To avoid any confusion, we may write $\mathbb{D}=\mathbb{D}^{\lambda}$. The equivalence $\Theta^{\lambda}$ intertwines the duality functors, i.e., we have

$$
\mathbb{D}^{\lambda+\mu} \circ \Theta^{\lambda}=\Theta^{\lambda} \circ \mathbb{D}^{\mu} .
$$

For any locally closed affine embedding of $T$-torsors $i: Z \rightarrow Y$ with $Z$ smooth, we define the functor

$$
i_{!}=\mathbb{D} \circ i_{\bullet} \circ \mathbb{D}: \mathbf{M}_{h}^{\tilde{\lambda}}(Z) \rightarrow \mathbf{M}_{h}^{\tilde{\lambda}}(Y) .
$$

As in Section 3.2, we have a morphism of exact functors $\psi: i_{!} \rightarrow i_{\bullet}$ which is an isomorphism if $i$ is a closed embedding. The intermediate extension functor $i_{\bullet}$ is defined in the same way.

Remark 3.4. Assume that $Z$ is smooth. Let $\mathscr{M} \in \mathbf{M}_{h}^{\lambda}(Z)$. Put $\mu=0$ in (3.10). Using the equivalence $\Phi$ we see that $\mathscr{M}^{\dagger}$ is a right module over the sheaf of algebras

$$
\mathscr{D}_{Z}^{\lambda}=\mathscr{L}_{Z}^{-\lambda} \otimes_{\mathscr{O}_{Z}} \mathscr{D}_{Z} \otimes_{\mathscr{O}_{Z}} \mathscr{L}_{Z}^{\lambda}
$$

Furthermore, we have

$$
\mathbb{D}(\mathscr{M})^{\dagger}=\Omega_{Z} \otimes_{\mathscr{O}_{Z}} \otimes_{\mathscr{O}_{Z}} \mathscr{L}_{Z}^{2 \lambda} \otimes_{\mathscr{O}_{Z}} \mathscr{E}_{x} t_{\mathscr{D}}^{\lambda} \operatorname{dim}^{\operatorname{dim} Z}\left(\mathscr{M}^{\dagger}, \mathscr{D}_{Z}^{\lambda}\right)
$$

by Lemma 3.3 and (3.11), compare [KT1, (2.1.2)].

3.6. Injective and projective limit of categories. Let us introduce the following notation. Let $A$ be a filtering poset. For any inductive system of categories $\left(\mathcal{C}_{\alpha}\right)_{\alpha \in A}$ with functors $i_{\alpha \beta}: \mathcal{C}_{\alpha} \rightarrow \mathcal{C}_{\beta}, \alpha \leqslant \beta$, we denote by $2 \lim _{\longrightarrow} \mathcal{C}_{\alpha}$ its inductive limit, i.e., the category whose objects are pairs $\left(\alpha, M_{\alpha}\right)$ with $\alpha \vec{\epsilon} A, M_{\alpha} \in \mathcal{C}_{\alpha}$ and

$$
\operatorname{Hom}_{2 \lim } \mathcal{C}_{\alpha}\left(\left(\alpha, M_{\alpha}\right),\left(\beta, N_{\beta}\right)\right)=\underset{\gamma \geqslant \alpha, \beta}{\lim _{\gamma}} \operatorname{Hom}_{\mathcal{C}_{\gamma}}\left(i_{\alpha \gamma}\left(M_{\alpha}\right), i_{\beta \gamma}\left(N_{\beta}\right)\right) .
$$

For any projective system of categories $\left(\mathcal{C}_{\alpha}\right)_{\alpha \in A}$ with functors $j_{\alpha \beta}: \mathcal{C}_{\beta} \rightarrow \mathcal{C}_{\alpha}$, $\alpha \leqslant \beta$, we denote by $2 \lim _{\mathcal{C}} \mathcal{C}_{\alpha}$ its projective limit, i.e., the category whose objects are systems consisting of objects $M_{\alpha} \in \mathcal{C}_{\alpha}$ given for all $\alpha \in A$ and isomorphisms $j_{\alpha \beta}\left(M_{\beta}\right) \rightarrow M_{\alpha}$ for each $\alpha \leqslant \beta$ and satisfying the compatibility condition for each $\alpha \leqslant \beta \leqslant \gamma$. Morphisms are defined in the obvious way. See e.g., [KV], 3.2, 3.3]. 
3.7. The $\mathscr{O}$-modules on ind-schemes. An ind-scheme $X$ is a filtering inductive system of schemes $\left(X_{\alpha}\right)_{\alpha \in A}$ with closed embeddings $i_{\alpha \beta}: X_{\alpha} \rightarrow X_{\beta}$ for $\alpha \leqslant \beta$ such that $X$ represents the ind-object "lim" $X_{\alpha}$. See [KS, 1.11] for details on indobjects. Below we will simply write $\lim$ for "lim", hoping this does not create

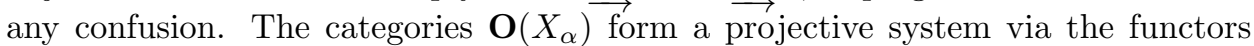
$i_{\alpha \beta}^{!}: \mathbf{O}\left(X_{\beta}\right) \rightarrow \mathbf{O}\left(X_{\alpha}\right)$. Following [BD, 7.11.4] and [KV, 3.3] we define the category of $\mathscr{O}$-modules on $X$ as

$$
\mathbf{O}(X)=2 \lim _{\longleftarrow} \mathbf{O}\left(X_{\alpha}\right) .
$$

It is an abelian category. An object $\mathscr{M}$ of $\mathbf{O}(X)$ is represented by

$$
\mathscr{M}=\left(\mathscr{M}_{\alpha}, \varphi_{\alpha \beta}: i_{\alpha \beta}^{!} \mathscr{M}_{\beta} \rightarrow \mathscr{M}_{\alpha}\right)
$$

where $\mathscr{M}_{\alpha}$ is an object of $\mathbf{O}\left(X_{\alpha}\right)$ and $\varphi_{\alpha \beta}, \alpha \leqslant \beta$, is an isomorphism in $\mathbf{O}\left(X_{\alpha}\right)$.

Note that any object $\mathscr{M}$ of $\mathbf{O}(X)$ is an inductive limit of objects from $\mathbf{O}\left(X_{\alpha}\right)$. More precisely, we first identify $\mathbf{O}\left(X_{\alpha}\right)$ as a full subcategory of $\mathbf{O}(X)$ in the following way: since the poset $A$ is filtering, to any $\mathscr{M}_{\alpha} \in \mathbf{O}\left(X_{\alpha}\right)$ we may associate a canonical object $\left(\mathscr{N}_{\beta}\right)$ in $\mathbf{O}(X)$ such that $\mathscr{N}_{\beta}=i_{\alpha \beta *}\left(\mathscr{M}_{\alpha}\right)$ for $\alpha \leqslant \beta$ and the structure isomorphisms $\varphi_{\beta \gamma}, \beta \leqslant \gamma$, are the obvious ones. Let us denote this object in $\mathbf{O}(X)$ again by $\mathscr{M}_{\alpha}$. Given any object $\mathscr{M} \in \mathbf{O}(X)$ represented by $\mathscr{M}=\left(\mathscr{M}_{\alpha}, \varphi_{\alpha \beta}\right)$, these $\mathscr{M}_{\alpha} \in \mathbf{O}(X), \alpha \in A$, form an inductive system via the canonical morphisms $\mathscr{M}_{\alpha} \rightarrow \mathscr{M}_{\beta}$. Then, the ind-object $\lim \mathscr{M}_{\alpha}$ of $\mathbf{O}(X)$ is represented by $\mathscr{M}$. So, we define the space of global sections of $\mathscr{\mathscr { M }}$ to be the inductive limit of vector spaces

$$
\Gamma(X, \mathscr{M})=\lim _{\longrightarrow} \Gamma\left(X_{\alpha}, \mathscr{M}_{\alpha}\right) .
$$

We will also use the category $\hat{\mathbf{O}}(X)$ defined as the limit of the projective system of categories $\left(\mathbf{O}\left(X_{\alpha}\right), i_{\alpha \beta}^{*}\right)$; see $\left.\mathrm{BD}, 7.11 .3\right]$ or $[\mathrm{KV}, 3.3]$. Note that the canonical isomorphisms $i_{\alpha \beta}^{*} \mathscr{O}_{X_{\beta}}=\mathscr{O}_{X_{\alpha}}$ yield an object $\left(\mathscr{O}_{X_{\alpha}}\right)_{\alpha \in A}$ in $\hat{\mathbf{O}}(X)$. We denote this object by $\mathscr{O}_{X}$. An object $\mathscr{F} \in \hat{\mathbf{O}}(X)$ is said to be flat if each $\mathscr{F}_{\alpha}$ is a flat $\mathscr{O}_{X_{\alpha}}$-module. Such a $\mathscr{F}$ yields an exact functor

$$
\mathbf{O}(X) \rightarrow \mathbf{O}(X), \quad \mathscr{M} \mapsto \mathscr{M} \otimes_{\mathscr{O}_{X}} \mathscr{F}=\left(\mathscr{M}_{\alpha} \otimes_{\mathscr{O}_{X_{\alpha}}} \mathscr{F}_{\alpha}\right)_{\alpha \in A}
$$

For $\mathscr{F} \in \hat{\mathbf{O}}(X)$ the vector spaces $\Gamma\left(X_{\alpha}, \mathscr{F}_{\alpha}\right)$ form a projective system with the structure maps induced by the functors $i_{\alpha \beta}^{*}$. We set

$$
\Gamma(X, \mathscr{F})=\lim _{\longleftarrow} \Gamma\left(X_{\alpha}, \mathscr{F}_{\alpha}\right) \text {. }
$$

3.8. The $\mathscr{D}$-modules on ind-schemes. The category of $\mathscr{D}$-modules on the indscheme $X$ is defined as the limit of the inductive system of categories $\left(\mathbf{M}\left(X_{\alpha}\right), i_{\alpha \beta} \bullet\right)$; see e.g., [KV] 3.3]. We will denote it by $\mathbf{M}(X)$. Since $\mathbf{M}\left(X_{\alpha}\right)$ are abelian categories and $i_{\alpha \beta}$ e are exact functors, the category $\mathbf{M}(X)$ is abelian. Recall that an object of $\mathbf{M}(X)$ is represented by a pair $\left(\alpha, \mathscr{M}_{\alpha}\right)$ with $\alpha \in A, \mathscr{M}_{\alpha} \in \mathbf{M}\left(X_{\alpha}\right)$. There is an exact and faithful forgetful functor

$$
\mathbf{M}(X) \rightarrow \mathbf{O}(X), \quad \mathscr{M}=\left(\alpha, \mathscr{M}_{\alpha}\right) \rightarrow \mathscr{M}^{\mathscr{O}}=\left(i_{\alpha \beta} \bullet\left(\mathscr{M}_{\alpha}\right)^{\mathscr{O}}\right)_{\beta \geqslant \alpha} .
$$

The global sections functor on $\mathbf{M}(X)$ is defined by

$$
\Gamma(X, \mathscr{M})=\Gamma\left(X, \mathscr{M}^{\mathscr{O}}\right) .
$$

Next, we say that $X$ is a $T$-ind-scheme if $X=\lim _{\longrightarrow} X_{\alpha}$ with each $X_{\alpha}$ being a $T$-scheme and $i_{\alpha \beta}: X_{\alpha} \rightarrow X_{\beta}$ being $T$-equivariant. We define $\mathbf{M}^{T}(X)$ to 
be the abelian category given by the limit of the inductive system of categories $\left(\mathbf{M}^{T}\left(X_{\alpha}\right), i_{\alpha \beta} \bullet\right)$. The functors (3.4) for each $X_{\alpha}$ yield an exact and faithful functor

$$
\mathbf{M}^{T}(X) \rightarrow \mathbf{M}(X) .
$$

The functor $\Gamma$ on $\mathbf{M}^{T}(X)$ is given by the functor $\Gamma$ on $\mathbf{M}(X)$.

Finally, given a $T$-ind-scheme $X=\lim _{\longrightarrow} X_{\alpha}$ let $\pi: X^{\dagger} \rightarrow X$ be a $T$-torsor over $X$, i.e., $\pi$ is the limit of an inductive system of $T$-torsors $\pi_{\alpha}: X_{\alpha}^{\dagger} \rightarrow X_{\alpha}$. We say that $\pi$ is admissible if each of the $\pi_{\alpha}$ is admissible. Assume this is the case. Then the categories $\mathbf{M}^{\lambda}\left(X_{\alpha}\right), \mathbf{M}^{\tilde{\lambda}}\left(X_{\alpha}\right)$ form, respectively, two inductive systems of categories via $i_{\alpha \beta}$. Let

$$
\mathbf{M}^{\lambda}(X)=2 \underset{\longrightarrow}{\lim } \mathbf{M}^{\lambda}\left(X_{\alpha}\right), \quad \mathbf{M}^{\tilde{\lambda}}(X)=2 \lim _{\longrightarrow} \mathbf{M}^{\tilde{\lambda}}\left(X_{\alpha}\right) .
$$

They are abelian subcategories of $\mathbf{M}^{T}\left(X^{\dagger}\right)$. For any object $\mathscr{M}=\left(\alpha, \mathscr{M}_{\alpha}\right)$ of $\mathbf{M}^{T}\left(X^{\dagger}\right)$, the $\mathscr{O}_{X_{\beta}}$-modules $\left(i_{\alpha \beta} \cdot \mathscr{M}_{\alpha}\right)^{\dagger}$ with $\beta \geqslant \alpha$ give an object of $\mathbf{O}(X)$. We will denote it by $\mathscr{M}^{\dagger}$. The functor

$$
\mathbf{M}^{T}\left(X^{\dagger}\right) \rightarrow \mathbf{O}(X), \quad \mathscr{M} \mapsto \mathscr{M}^{\dagger}
$$

is exact and faithful. For $\mathscr{M} \in \mathbf{M}^{\tilde{\lambda}}(X)$ we will write

$$
\Gamma(\mathscr{M})=\Gamma\left(X, \mathscr{M}^{\dagger}\right) \text {. }
$$

Note that it is also equal to $\Gamma\left(X^{\dagger}, \mathscr{M}\right)^{T}$. We will consider the following categories

$$
\mathbf{M}_{h}^{\tilde{\lambda}}(X)=2 \underset{\lim }{ } \mathbf{M}_{h}^{\tilde{\lambda}}\left(X_{\alpha}\right), \quad \mathbf{M}_{h}^{\lambda}(X)=2 \lim _{\longrightarrow} \mathbf{M}_{h}^{\lambda}\left(X_{\alpha}\right) .
$$

Let $Y$ be a smooth scheme. A locally closed affine embedding $i: Y \rightarrow X$ is the composition of an affine open embedding $i_{1}: Y \rightarrow \bar{Y}$ with a closed embedding $i_{2}: \bar{Y} \rightarrow X$. For such a morphism the functor $i_{\bullet}: \mathbf{M}_{h}^{\tilde{\lambda}}(Y) \rightarrow \mathbf{M}_{h}^{\tilde{\lambda}}(X)$ is defined by $i_{\bullet}=i_{2} \bullet i_{1}$, and the functor $i_{!}: \mathbf{M}_{h}^{\tilde{\lambda}}(Y) \rightarrow \mathbf{M}_{h}^{\tilde{\lambda}}(X)$ is defined by $i_{!}=i_{2} \circ i_{1 !}$.

3.9. The sheaf of differential operators on a formally smooth ind-scheme. Let $X$ be a formally smooth 1 ind-scheme. Fix $\beta \geqslant \alpha$ in $A$. Let $\mathscr{D}_{\text {iff }}{ }_{\beta, \alpha}$ be the $\mathscr{O}_{X_{\beta} \times X_{\alpha}}$-submodule of $\mathscr{H}_{0} m_{\mathbb{C}}\left(\mathscr{O}_{X_{\beta}}, i_{\alpha \beta *} \mathscr{O}_{X_{\alpha}}\right)$ consisting of local sections supported set-theoretically on the diagonal $X_{\alpha} \subset X_{\beta} \times X_{\alpha}$. Here $\mathscr{H}_{0 m_{\mathbb{C}}}\left(\mathscr{O}_{X_{\beta}}, i_{\alpha \beta *} \mathscr{O}_{X_{\alpha}}\right)$ denotes the sheaf of morphisms between the sheaves of $\mathbb{C}$-vector spaces associated with $\mathscr{O}_{X_{\beta}}$ and $i_{\alpha \beta *} \mathscr{O}_{X_{\alpha}}$. As a left $\mathscr{O}_{X_{\alpha}}$-module $\mathscr{D}_{i f f}{ }_{\beta, \alpha}$ is quasi-coherent; see e.g., [BB, Section 1.1]. So it is an object in $\mathbf{O}\left(X_{\alpha}\right)$. For $\beta \leqslant \gamma$ the functor $i_{\beta \gamma *}$ and the canonical map $\mathscr{O}_{X_{\gamma}} \rightarrow i_{\beta \gamma *} \mathscr{O}_{X_{\beta}}$ yield a morphism of $\mathscr{O}_{X_{\alpha}}$-modules

$$
\mathscr{H}_{0} m_{\mathbb{C}}\left(\mathscr{O}_{X_{\beta}}, i_{\alpha \beta *} \mathscr{O}_{X_{\alpha}}\right) \rightarrow \mathscr{H}_{0 m_{\mathbb{C}}}\left(\mathscr{O}_{X_{\gamma}}, i_{\alpha \gamma *} \mathscr{O}_{X_{\alpha}}\right) \text {. }
$$

It induces a morphism $\mathscr{D}_{i f f}{ }_{\beta, \alpha} \rightarrow \mathscr{D}_{\text {iff }}{ }_{\gamma, \alpha}$ in $\mathbf{O}\left(X_{\alpha}\right)$. The $\mathscr{O}_{X_{\alpha}}$-modules $\mathscr{D i f f}_{\beta, \alpha}$, $\beta \geqslant \alpha$, together with these maps form an inductive system. Let

$$
\text { Diff }_{\alpha}=\underset{\beta \geqslant \alpha}{\lim _{\beta}} \mathscr{D i f f}_{\beta, \alpha} \in \mathbf{O}\left(X_{\alpha}\right) \text {. }
$$

The system consisting of the $\mathscr{D i f f}_{\alpha}$ 's and the canonical isomorphisms $i_{\alpha \beta}^{*} \mathscr{D}_{\text {iff }} \rightarrow$ $\mathscr{D i f f}_{\alpha}$ is a flat object in $\hat{\mathbf{O}}(X)$; see $\mathrm{BD}$, 7.11.11]. We will call it the sheaf of differential operators on $X$ and denote it by $\mathscr{D}_{X}$. It carries canonically a structure of $\mathscr{O}_{X}$-bimodules, and a structure of algebra given by

$$
\mathscr{D i f f}_{\gamma, \beta} \otimes_{\mathscr{O}_{X_{\beta}}} \mathscr{D}_{i f f}{ }_{\beta, \alpha} \rightarrow \operatorname{Diff}_{\gamma, \alpha}, \quad(g, f) \mapsto g \circ f, \quad \alpha \leqslant \beta \leqslant \gamma .
$$

\footnotetext{
${ }^{1}$ See [BD 7.11.1] and the references there for the definition of formally smooth.
} 
Any object $\mathscr{M} \in \mathbf{M}(X)$ admits a canonical right $\mathscr{D}_{X}$-action given by a morphism

$$
\mathscr{M} \otimes_{\mathscr{O}_{X}} \mathscr{D}_{X} \rightarrow \mathscr{M}
$$

in $\mathbf{O}(X)$ which is compatible with the multiplication in $\mathscr{D}_{X}$.

\section{Localization theorem for AFFine Lie ALGEBRAs of NEgAtive LEvel}

In this section we first consider the affine localization theorem which relates right $\mathscr{D}$-modules on the affine flag variety (an ind-scheme) to a category of modules over the affine Lie algebra with integral weights and a negative level. Then we compute the $\mathscr{D}$-modules corresponding to Verma and parabolic Verma modules. All the constructions here hold for a general simple linear group. We will only use the case of $S L_{m}$, since the multiplicities on the left hand side of (2.11) that we want to compute are the same for $\mathfrak{s l}_{m}$ and $\mathfrak{g l}_{m}$. We will use, for $\mathfrak{s l}_{m}$, the same notation as in Section 2 for $\mathfrak{g l}_{m}$. In particular $\mathfrak{g}_{0}=\mathfrak{s l}_{m}$ and $\mathfrak{t}_{0}^{*}$ is now given the basis consisting of the weights $\epsilon_{i}-\epsilon_{i+1}$ with $1 \leqslant i \leqslant m-1$. We identify $\mathcal{P}_{n}$ as a subset of $\mathfrak{t}_{0}^{*}$ via the map

$$
\mathcal{P}_{n} \rightarrow \mathfrak{t}_{0}^{*}, \quad \lambda=\left(\lambda_{1}, \ldots, \lambda_{m}\right) \mapsto \sum_{i=1}^{m}\left(\lambda_{i}-n / m\right) \epsilon_{i} .
$$

Finally, we will modify slightly the definition of $\mathfrak{g}$ by extending $\mathbb{C}\left[t, t^{-1}\right]$ to $\mathbb{C}((t))$, i.e., from now on we set

$$
\mathfrak{g}=\mathfrak{g}_{0} \otimes \mathbb{C}((t)) \oplus \mathbb{C} \mathbf{1} \oplus \mathbb{C} \partial .
$$

The bracket is given in the same way as before. We will again denote by $\mathfrak{b}, \mathfrak{n}, \mathfrak{q}$, etc., the corresponding Lie subalgebras of $\mathfrak{g}$.

4.1. The affine Kac-Moody group. Consider the group ind-scheme $L G_{0}=$ $G_{0}(\mathbb{C}((t)))$ and the group scheme $L^{+} G_{0}=G_{0}(\mathbb{C}[[t]])$. Let $I \subset L^{+} G_{0}$ be the Iwahori subgroup. It is the preimage of $B_{0}$ via the canonical map $L^{+} G_{0} \rightarrow G_{0}$. For $z \in \mathbb{C}^{*}$ the loop rotation $t \mapsto z t$ yields a $\mathbb{C}^{*}$-action on $L G_{0}$. Write

$$
\widehat{L G}_{0}=\mathbb{C}^{*} \ltimes L G_{0} .
$$

Let $G$ be the Kac-Moody group associated with $\mathfrak{g}$. It is a group ind-scheme which is a central extension

$$
1 \rightarrow \mathbb{C}^{*} \rightarrow G \rightarrow \widehat{L G}_{0} \rightarrow 1
$$

see e.g., $\mathrm{Ku} 2$, Section 13.2]. There is an obvious projection $\mathrm{pr}: G \rightarrow L G_{0}$. We set

$$
B=\operatorname{pr}^{-1}(I), \quad Q=\operatorname{pr}^{-1}\left(L^{+} G_{0}\right), \quad T=\operatorname{pr}^{-1}\left(T_{0}\right) .
$$

Finally, let $N$ be the prounipotent radical of $B$. We have

$$
\mathfrak{g}=\operatorname{Lie}(G), \quad \mathfrak{b}=\operatorname{Lie}(B), \quad \mathfrak{q}=\operatorname{Lie}(Q), \quad \mathfrak{t}=\operatorname{Lie}(T), \quad \mathfrak{n}=\operatorname{Lie}(N) .
$$

4.2. The affine flag variety. Let $X=G / B$ be the affine flag variety. It is a formally smooth ind-scheme. The enhanced affine flag variety $X^{\dagger}=G / N$ is a $T$-torsor over $X$ via the canonical projection

$$
\pi: X^{\dagger} \rightarrow X
$$

The $T$-action on $X^{\dagger}$ is given by $g N \mapsto g h^{-1} N$ for $h \in T, g \in G$. The $T$-torsor $\pi$ is admissible; see the end of Section A.1 The ind-scheme $X^{\dagger}$ is also formally smooth. 
For any subscheme $Z$ of $X$ we will write $Z^{\dagger}=\pi^{-1}(Z)$. The $B$-orbit decomposition of $X$ is

$$
X=\bigsqcup_{w \in \mathfrak{S}} X_{w}, \quad X_{w}=B \dot{w} B / B
$$

where $\dot{w}$ is a representative of $w$ in the normalizer of $T$ in $G$. Each $X_{w}$ is an affine space of dimension $l(w)$. Its closure $\bar{X}_{w}$ is an irreducible projective variety. We have

$$
\bar{X}_{w}=\bigsqcup_{w^{\prime} \leqslant w} X_{w^{\prime}}, \quad X=\underset{w}{\lim } \bar{X}_{w} .
$$

4.3. Localization theorem. Recall the sheaf of differential operators $\mathscr{D}_{X^{\dagger}} \in$ $\hat{\mathbf{O}}\left(X^{\dagger}\right)$. The space of sections of $\mathscr{D}_{X^{\dagger}}$ is defined as in (3.14). The left action of $G$ on $X^{\dagger}$ yields an algebra homomorphism

$$
\delta_{l}: \mathcal{U}(\mathfrak{g}) \rightarrow \Gamma\left(X^{\dagger}, \mathscr{D}_{X^{\dagger}}\right) .
$$

Since the $G$-action on $X^{\dagger}$ commutes with the right $T$-action, the image of the map above lies in the $T$-invariant part of $\Gamma\left(X^{\dagger}, \mathscr{D}_{X^{\dagger}}\right)$. So for $\mathscr{M} \in \mathbf{M}^{T}\left(X^{\dagger}\right)$ the $\mathscr{D}_{X^{\dagger-}}$ action on $\mathscr{M}$ given by (3.17) induces a $\mathfrak{g}$-action 2 on $\mathscr{M}^{\dagger}$ via $\delta_{l}$. In particular the vector space $\Gamma(\mathscr{M})$ as defined in (3.16) is a $\mathfrak{g}$-module. Let $\mathbf{M}(\mathfrak{g})$ be the category of $\mathfrak{g}$-modules. We say that a weight $\lambda \in \mathfrak{t}^{*}$ is antidominant (resp. dominant, regular) if for any $\alpha \in \Pi^{+}$we have $\langle\lambda: \alpha\rangle \leqslant 0$ (resp. $\langle\lambda: \alpha\rangle \geqslant 0,\langle\lambda: \alpha\rangle \neq 0$ ).

Proposition 4.1. (a) The functor

$$
\Gamma: \mathbf{M}^{\lambda}(X) \rightarrow \mathbf{M}(\mathfrak{g}), \quad \mathscr{M} \mapsto \Gamma(\mathscr{M})
$$

is exact if $\lambda+\rho$ is antidominant.

(b) The functor

$$
\Gamma: \mathbf{M}^{\tilde{\lambda}}(X) \rightarrow \mathbf{M}(\mathfrak{g}), \quad \mathscr{M} \mapsto \Gamma(\mathscr{M})
$$

is exact if $\lambda+\rho$ is antidominant.

Proof. A proof of part (a) is sketched in $\mathrm{BD}$, Theorem 7.15.6]. A detailed proof can be given using similar techniques as in the proof of the Proposition A.2 below. This is left to the reader. See also [FG. Theorem 2.2] for another proof of this result. Now, let us concentrate on part (b). Let $\mathscr{M}=\left(\alpha, \mathscr{M}_{\alpha}\right)$ be an object in $\mathbf{M}^{\tilde{\lambda}}(X)$. By definition the action of $\mathfrak{m}_{\lambda}$ on $\mathscr{M}^{\dagger}$ is nilpotent. Let $\mathscr{M}_{n}$ be the maximal subobject of $\mathscr{M}$ such that the ideal $\left(\mathfrak{m}_{\lambda}\right)^{n}$ acts on $\mathscr{M}_{n}^{\dagger}$ by zero. We have $\mathscr{M}_{n-1} \subset \mathscr{M}_{n}$ and $\mathscr{M}=\lim _{n} \mathscr{M}_{n}$. Write $R^{k} \Gamma(X,-)$ for the $k$-th derived functor of the global sections functor $\vec{\Gamma}(X,-)$. Given $n \geqslant 1$, suppose that

$$
R^{k} \Gamma\left(X, \mathscr{M}_{n}^{\dagger}\right)=0, \quad \forall k>0 .
$$

Since $\mathscr{M}_{n+1} / \mathscr{M}_{n}$ is an object of $\mathbf{M}^{\lambda}(X)$, by part (a) we have

$$
R^{k} \Gamma\left(X,\left(\mathscr{M}_{n+1} / \mathscr{M}_{n}\right)^{\dagger}\right)=0, \quad \forall k>0 .
$$

The long exact sequence for $R \Gamma(X,-)$ applied to the short exact sequence

$$
0 \longrightarrow \mathscr{M}_{n}^{\dagger} \longrightarrow \mathscr{M}_{n+1}^{\dagger} \longrightarrow\left(\mathscr{M}_{n+1} / \mathscr{M}_{n}\right)^{\dagger} \longrightarrow 0
$$

\footnotetext{
${ }^{2}$ More precisely, here by $\mathfrak{g}$-action we mean the $\mathfrak{g}$-action on the associated sheaf of vector spaces $\left(\mathscr{M}^{\dagger}\right)^{\mathbb{C}}$; see Step 1 of the proof of Proposition A.2 for details.
} 
implies that $R^{k} \Gamma\left(X, \mathscr{M}_{n+1}^{\dagger}\right)=0$ for any $k>0$. Therefore by induction the vector space $R^{k} \Gamma\left(X, \mathscr{M}_{n}^{\dagger}\right)$ vanishes for any $n \geqslant 1$ and $k>0$. Finally, since the functor $R^{k} \Gamma(X,-)$ commutes with direct limits (see e.g., [TT, Lemma B.6]), we have

$$
R^{k} \Gamma\left(X, \mathscr{M}^{\dagger}\right)=\lim _{\longrightarrow} R^{k} \Gamma\left(X, \mathscr{M}_{n}^{\dagger}\right)=0, \quad \forall k>0 .
$$

4.4. The category $\tilde{\mathcal{O}}_{\kappa}$ and Verma modules. For a $\mathfrak{t}$-module $M$ and $\lambda \in \mathfrak{t}^{*}$ let

$$
M_{\tilde{\lambda}}=\left\{m \in M \mid(h-\lambda(h))^{N} m=0, \forall h \in \mathfrak{t}, N \gg 0\right\} .
$$

We call a $\mathrm{t}$-module $M$ a generalized weight module if it satisfies the conditions

$$
\begin{gathered}
M=\bigoplus_{\lambda \epsilon_{\kappa} \mathfrak{t}^{*}} M_{\tilde{\lambda}}, \\
\operatorname{dim}_{\mathbb{C}} M_{\tilde{\lambda}}<\infty, \quad \forall \lambda \in \mathfrak{t}^{*} .
\end{gathered}
$$

Its character $\operatorname{ch}(M)$ is defined as the formal sum

$$
\operatorname{ch}(M)=\sum_{\lambda \in \mathfrak{t}^{*}} \operatorname{dim}_{\mathbb{C}}\left(M_{\tilde{\lambda}}\right) e^{\lambda} .
$$

Let $\tilde{\mathcal{O}}$ be the category consisting of the $\mathcal{U}(\mathfrak{g})$-modules $M$ such that

- as a $\mathfrak{t}$-module $M$ is a generalized weight module,

- there exists a finite subset $\Xi \subset \mathfrak{t}^{*}$ such that $M_{\tilde{\lambda}} \neq 0$ implies that $\lambda \in$ $\Xi+\sum_{i=0}^{m-1} \mathbb{Z}_{\leqslant 0} \alpha_{i}$.

The category $\tilde{\mathcal{O}}$ is abelian. We define the duality functor $\mathbf{D}$ on $\tilde{\mathcal{O}}$ by

$$
\mathbf{D} M=\bigoplus_{\lambda \in \mathfrak{t}^{*}} \operatorname{Hom}\left(M_{\tilde{\lambda}}, \mathbb{C}\right)
$$

with the action of $\mathfrak{g}$ given by the involution $\sigma$; see Section 2.3 . Let $\tilde{\mathcal{O}}_{\kappa}$ be the full subcategory of $\tilde{\mathcal{O}}$ consisting of the $\mathfrak{g}$-modules on which $\mathbf{1}-(\kappa-m)$ acts locally nilpotently. The category $\tilde{\mathcal{O}}_{\kappa}$ is also abelian. It is stable under the duality functor, because $\sigma(\mathbf{1})=\mathbf{1}$. The category $\mathcal{O}_{\kappa}$ is a Serre subcategory of $\tilde{\mathcal{O}}_{\kappa}$. For $\lambda \in{ }_{\kappa} \mathbf{t}^{*}$ we consider the Verma module

$$
N_{\kappa}(\lambda)=\mathcal{U}(\mathfrak{g}) \otimes_{\mathcal{U}(\mathfrak{b})} \mathbb{C}_{\lambda} .
$$

Here $\mathbb{C}_{\lambda}$ is the one-dimensional $\mathfrak{b}$-module such that $\mathfrak{n}$ acts trivially and $\mathfrak{t}$ acts by $\lambda$. It is an object of $\tilde{\mathcal{O}}_{\kappa}$. Let $L_{\kappa}(\lambda)$ be the unique simple quotient of $N_{\kappa}(\lambda)$. We have $\mathbf{D} L_{\kappa}(\lambda)=L_{\kappa}(\lambda)$ for any $\lambda$. A simple subquotient of a module $M \in \tilde{\mathcal{O}}_{\kappa}$ is isomorphic to $L_{\kappa}(\lambda)$ for some $\lambda \in \kappa_{\kappa}{ }^{*}$. The classes $\left[L_{\kappa}(\lambda)\right]$ form a basis of the vector space $\left[\tilde{\mathcal{O}}_{\kappa}\right]$, because the characters of the $L_{\kappa}(\lambda)$ 's are linearly independent.

Denote by $\Lambda$ the set of integral weights in ${ }_{\kappa} \mathrm{t}^{*}$. Let $\lambda \in \Lambda$ and $w \in \mathfrak{S}$. Recall the line bundle $\mathscr{L}_{X_{w}}^{\lambda}$ from Section 3.5. Let

$$
\mathscr{A}_{w}^{\lambda}=\Omega_{X_{w}^{\dagger}} \otimes_{\mathscr{O}_{X_{w}^{\dagger}}^{\dagger}} \pi^{*}\left(\mathscr{L}_{X_{w}}^{\lambda}\right) .
$$

It is an object of $\mathbf{M}_{h}^{\lambda}\left(X_{w}\right)$ and

$$
\mathbb{D}\left(\mathscr{A}_{w}^{\lambda}\right)=\mathscr{A}_{w}^{\lambda} .
$$

Let $i_{w}: X_{w}^{\dagger} \rightarrow X^{\dagger}$ be the canonical embedding. It is locally closed and affine. We have the following objects in $\mathbf{M}_{h}^{\lambda}(X)$,

$$
\mathscr{A}_{w !}^{\lambda}=i_{w !}\left(\mathscr{A}_{w}^{\lambda}\right), \quad \mathscr{A}_{w ! \bullet}^{\lambda}=i_{w ! \bullet}\left(\mathscr{A}_{w}^{\lambda}\right), \quad \mathscr{A}_{w \bullet}^{\lambda}=i_{w \bullet}\left(\mathscr{A}_{w}^{\lambda}\right)
$$


We will consider the Serre subcategory $\mathbf{M}_{0}^{\lambda}(X)$ of $\mathbf{M}_{h}^{\lambda}(X)$ generated by the simple objects $\mathscr{A}_{w ! \bullet}^{\lambda}$ for $w \in \mathfrak{S}$. It is an artinian category. Since $\mathbb{D}\left(\mathscr{A}_{w ! \bullet}^{\lambda}\right)=\mathscr{A}_{w ! \bullet}^{\lambda}$, the category $\mathbf{M}_{0}^{\lambda}(X)$ is stable under the duality. We have the following proposition.

Proposition 4.2. Let $\lambda \in \Lambda$ such that $\lambda+\rho$ is antidominant. Then

(a) $\Gamma\left(\mathscr{A}_{w !}^{\lambda}\right)=N_{\kappa}(w \cdot \lambda)$,

(b) $\Gamma\left(\mathscr{A}_{w \bullet}^{\lambda}\right)=\mathbf{D} N_{\kappa}(w \cdot \lambda)$,

(c) $\Gamma\left(\mathscr{A}_{w ! \bullet}^{\lambda}\right)= \begin{cases}L_{\kappa}(w \cdot \lambda) & \text { if } w \text { is the shortest element in } w \mathfrak{S}(\lambda), \\ 0 & \text { else. }\end{cases}$

The proof of the proposition will be given in Appendix A. It relies on results of Kashiwara-Tanisaki [KT1] and uses translation functors for the affine category $\mathcal{O}$.

4.5. The parabolic Verma modules. Let ${ }^{Q_{\mathfrak{S}}}$ be the set of the longest representatives of the cosets $\mathfrak{S}_{0} \backslash \mathfrak{S}$. Let $w_{0}$ be the longest element in $\mathfrak{S}_{0}$. Recall the following basic facts.

Lemma 4.3. For $w \in \mathfrak{S}$ if $w \cdot \lambda \in \Lambda^{+}$for some $\lambda \in \Lambda$ with $\lambda+\rho$ antidominant, then $w \in{ }^{Q} \mathfrak{S}$. Further, if $w \in{ }^{Q} \mathfrak{S}$ then we have

(a) the element $w$ is the unique element $v$ in $\mathfrak{S}_{0} w$ such that $\Pi_{0}^{+} \subset-v\left(\Pi^{+}\right)$,

(b) for any $v \in \mathfrak{S}_{0}$ we have $l(v w)=l(w)-l(v)$,

(c) the element $w_{0} w$ is the shortest element in $\mathfrak{S}_{0} w$.

The $Q$-orbit decomposition of $X$ is given by

$$
X=\bigsqcup_{w \in Q \mathscr{C}} Y_{w}, \quad Y_{w}=Q \dot{w} B / B
$$

Each $Y_{w}$ is a smooth subscheme of $X$, and $X_{w}$ is open and dense in $Y_{w}$. The closure of $Y_{w}$ in $X$ is an irreducible projective variety of dimension $l(w)$ given by

$$
\bar{Y}_{w}=\bigsqcup_{w^{\prime} \in Q \mathfrak{S}, w^{\prime} \leqslant w} Y_{w^{\prime}} .
$$

Recall that $Y_{w}^{\dagger}=\pi^{-1}\left(Y_{w}\right)$. The canonical embedding $j_{w}: Y_{w}^{\dagger} \rightarrow X^{\dagger}$ is locally closed and affine; see Remark 5.2(b). For $\lambda \in \Lambda$ and $w \in{ }^{Q} \mathfrak{S}$ let

$$
\mathscr{B}_{w}^{\lambda}=\Omega_{Y_{w}^{\dagger}} \otimes_{\mathscr{O}_{Y_{w}^{\dagger}}} \pi^{*}\left(\mathscr{L}_{Y_{w}}^{\lambda}\right) \text {. }
$$

We have the following objects in $\mathbf{M}_{h}^{\lambda}(X)$

$$
\mathscr{B}_{w !}^{\lambda}=j_{w !}\left(\mathscr{B}_{w}^{\lambda}\right), \quad \mathscr{B}_{w ! \bullet}^{\lambda}=j_{w ! \bullet}\left(\mathscr{B}_{w}^{\lambda}\right), \quad \mathscr{B}_{w \bullet}^{\lambda}=j_{w \bullet}\left(\mathscr{B}_{w}^{\lambda}\right) .
$$

Now, consider the canonical embedding $r: X_{w}^{\dagger} \rightarrow Y_{w}^{\dagger}$. Since $r$ is open and affine, we have $r^{*}=r^{!}$and the functors

$$
\left(r_{!}, r^{!}=r^{*}, r_{\bullet}\right)
$$

form a triple of adjoint functors between the categories $\mathbf{M}_{h}^{\tilde{\lambda}}\left(Y_{w}\right)$ and $\mathbf{M}_{h}^{\tilde{\lambda}}\left(X_{w}\right)$. Note that $r^{*}\left(\mathscr{B}_{w}^{\lambda}\right) \cong \mathscr{A}_{w}^{\lambda}$.

Lemma 4.4. For $\lambda \in \Lambda$ and $w \in Q_{\mathfrak{S}}$ the following holds.

(a) The adjunction map $r_{!} r^{*} \rightarrow$ Id yields a surjective morphism in $\mathbf{M}_{h}^{\lambda}\left(Y_{w}\right)$,

$$
r_{!}\left(\mathscr{A}_{w}^{\lambda}\right) \rightarrow \mathscr{B}_{w}^{\lambda} \text {. }
$$

(b) The adjunction map $\mathrm{Id} \rightarrow r_{\bullet} r^{*}$ yields an injective morphism in $\mathbf{M}_{h}^{\lambda}\left(Y_{w}\right)$,

$$
\mathscr{B}_{w}^{\lambda} \rightarrow r_{\bullet}\left(\mathscr{A}_{w}^{\lambda}\right) \text {. }
$$


Proof. We will only prove part (b). Part (a) follows from (b) by applying the duality functor $\mathbb{D}$. To prove (b), it is enough to show that the $\mathscr{O}_{Y_{w}^{\dagger}}$-module morphism

$$
\left(\mathscr{B}_{w}^{\lambda}\right)^{\mathscr{O}} \rightarrow\left(r \bullet r^{*}\left(\mathscr{B}_{w}^{\lambda}\right)\right)^{\mathscr{O}}
$$

is injective. Since $r$ is an open embedding, the right hand side is equal to $r_{*} r^{*}\left(\left(\mathscr{B}_{w}^{\lambda}\right)^{\mathscr{O}}\right)$. Now, consider the closed embedding

$$
i: Y_{w}^{\dagger}-X_{w}^{\dagger} \longrightarrow Y_{w}^{\dagger} .
$$

The morphism (4.10) can be completed into the following exact sequence in $\mathbf{O}\left(Y_{w}^{\dagger}\right)$,

$$
0 \rightarrow i_{*} i^{!}\left(\left(\mathscr{B}_{w}^{\lambda}\right)^{\mathscr{O}}\right) \rightarrow\left(\mathscr{B}_{w}^{\lambda}\right)^{\mathscr{O}} \rightarrow r_{*} r^{*}\left(\left(\mathscr{B}_{w}^{\lambda}\right)^{\mathscr{O}}\right)
$$

see e.g., [HTT, Proposition 1.7.1]. The $\mathscr{O}_{Y_{w}^{\dagger}}$-module $\left(\mathscr{B}_{w}^{\lambda}\right)^{\mathscr{O}}$ is locally free. So it has no subsheaf supported on the closed subscheme $Y_{w}^{\dagger}-X_{w}^{\dagger}$. We deduce that $i_{*} i^{!}\left(\left(\mathscr{B}_{w}^{\lambda}\right)^{\mathscr{O}}\right)=0$. Hence the morphism (4.10) is injective.

Lemma 4.5. For $\lambda \in \Lambda$ and $w \in{ }^{Q} \mathfrak{S}$ we have

$$
\mathscr{A}_{w ! \bullet}^{\lambda} \cong \mathscr{B}_{w ! \bullet}^{\lambda} \text {. }
$$

Proof. By applying the exact functor $j_{w}$ to the map (4.9) we see that $\mathscr{B}_{w \bullet}^{\lambda}$ is a subobject of $\mathscr{A}_{w \bullet}^{\lambda}$ in $\mathbf{M}_{h}^{\lambda}(X)$. In particular $\mathscr{B}_{w ! \bullet}^{\lambda}$ is a simple subobject of $\mathscr{A}_{w \bullet}^{\lambda}$; so it is isomorphic to $\mathscr{A}_{w ! \bullet}^{\lambda}$.

Proposition 4.6. Let $\lambda \in \Lambda$ such that $\lambda+\rho$ is antidominant, and let $w \in{ }^{Q} \mathfrak{S}$.

(a) If there exists $\alpha \in \Pi_{0}^{+}$such that $\langle w(\lambda+\rho): \alpha\rangle=0$, then

$$
\Gamma\left(\mathscr{B}_{w !}^{\lambda}\right)=0 .
$$

(b) We have

$$
\langle w(\lambda+\rho): \alpha\rangle \neq 0, \quad \forall \alpha \in \Pi_{0}^{+} \quad \Longleftrightarrow w \cdot \lambda \in \Lambda^{+} .
$$

In this case, we have

$$
\Gamma\left(\mathscr{B}_{w !}^{\lambda}\right)=M_{\kappa}(w \cdot \lambda), \quad \Gamma\left(\mathscr{B}_{w \bullet}^{\lambda}\right)=\mathbf{D} M_{\kappa}(w \cdot \lambda) .
$$

(c) We have

$$
\Gamma\left(\mathscr{B}_{w ! \bullet}^{\lambda}\right)= \begin{cases}L_{\kappa}(w \cdot \lambda) & \text { if } w \text { is the shortest element in } w \mathfrak{S}(\lambda), \\ 0 & \text { else. }\end{cases}
$$

Proof. The proof is inspired by the proof in the finite type case; see e.g., M, Theorem G.2.10]. First, by Kazhdan-Lusztig's algorithm (see Remark 6.3), the following equality holds in $\left[\mathbf{M}_{0}^{\lambda}(X)\right]$ :

$$
\left[\mathscr{B}_{w !}^{\lambda}\right]=\sum_{y \in \mathfrak{S}_{0}}(-1)^{l(y)}\left[\mathscr{A}_{y w !}^{\lambda}\right]
$$

Since $\lambda+\rho$ is antidominant, the functor $\Gamma$ is exact on $\mathbf{M}_{0}^{\lambda}(X)$ by Proposition 4.1(a). Therefore we have the following equalities in $\left[\tilde{\mathcal{O}}_{\kappa}\right]$ :

$$
\begin{aligned}
{\left[\Gamma\left(\mathscr{B}_{w !}^{\lambda}\right)\right] } & =\sum_{y \in \mathfrak{S}_{0}}(-1)^{l(y)}\left[\Gamma\left(\mathscr{A}_{y w !}^{\lambda}\right)\right] \\
& =\sum_{y \in \mathfrak{S}_{0}}(-1)^{l(y)}\left[N_{\kappa}(y w \cdot \lambda)\right] .
\end{aligned}
$$


Here the second equality is given by Proposition 4.2, Now, suppose that there exists $\alpha \in \Pi_{0}^{+}$such that $\langle w(\lambda+\rho): \alpha\rangle=0$. Let $s_{\alpha}$ be the corresponding reflection in $\mathfrak{S}_{0}$. Then we have

$$
s_{\alpha} w \cdot \lambda=w \cdot \lambda \text {. }
$$

By Lemma 4.3(b) we have $l(w)=l\left(s_{\alpha} w\right)+1$. So the right hand side of (4.12) vanishes. Therefore we have $\Gamma\left(\mathscr{B}_{w !}^{\lambda}\right)=0$. This proves part (a). Now, let us concentrate on part (b). Note that $\lambda+\rho$ is antidominant. Thus by Lemma 4.3(a) we have $\langle w(\lambda+\rho): \alpha\rangle \in \mathbb{N}$ for any $\alpha \in \Pi_{0}^{+}$. Hence

$$
\langle w(\lambda+\rho): \alpha\rangle \neq 0 \Longleftrightarrow\langle w(\lambda+\rho): \alpha\rangle \geqslant 1 \Longleftrightarrow\langle w \cdot \lambda: \alpha\rangle \geqslant 0 .
$$

By consequence $\langle w(\lambda+\rho): \alpha\rangle \neq 0$ for all $\alpha \in \Pi_{0}^{+}$if and only if $w \cdot \lambda$ belongs to $\Lambda^{+}$. In this case, the right hand side of (4.12) is equal to $\left[M_{\kappa}(w \cdot \lambda)\right]$ by the BGG-resolution. We deduce that

$$
\left[\Gamma\left(\mathscr{B}_{w !}^{\lambda}\right)\right]=\left[M_{\kappa}(w \cdot \lambda)\right] .
$$

Now, applying the exact functor $j_{\text {! }}$ to the surjective morphism in (4.8) yields a quotient map $\mathscr{B}_{w !}^{\lambda} \rightarrow \mathscr{A}_{w !}^{\lambda}$ in $\mathbf{M}^{\lambda}(X)$. The exactness of $\Gamma$ implies that $\Gamma\left(\mathscr{B}_{w !}^{\lambda}\right)$ is a quotient of $N_{\kappa}(w \cdot \lambda)=\Gamma\left(\mathscr{A}_{w !}^{\lambda}\right)$. Since $M_{\kappa}(w \cdot \lambda)$ is the maximal q-locally-finite quotient of $N_{\kappa}(w \cdot \lambda)$ and $\Gamma\left(\mathscr{B}_{w !}^{\lambda}\right)$ is q-locally finite, we deduce that $\Gamma\left(\mathscr{B}_{w !}^{\lambda}\right)$ is a quotient of $M_{\kappa}(w \cdot \lambda)$. So the first equality in part (b) follows from (4.13). The proof of the second one is similar. Finally, part (c) follows from Lemma 4.5] and Proposition 4.2

Remark 4.7. Note that if $w \in Q_{\mathfrak{S}}$ is a shortest element in $w \mathfrak{S}(\lambda)$, then we have $\langle w(\lambda+\rho): \alpha\rangle \neq 0$ for all $\alpha \in \Pi_{0}^{+}$. Indeed, if there exists $\alpha \in \Pi_{0}^{+}$such that $\langle w(\lambda+\rho): \alpha\rangle=0$. Let $s^{\prime}=w^{-1} s_{\alpha} w$. Then $s^{\prime}$ belongs to $\mathfrak{S}(\lambda)$. Therefore we have $l\left(w s^{\prime}\right)>l(w)$. But $w s^{\prime}=s_{\alpha} w$ and $s_{\alpha} \in \mathfrak{S}_{0}$, by Lemma 4.3 we have $l\left(w s^{\prime}\right)=$ $l\left(s_{\alpha} w\right)<l(w)$. This is a contradiction.

\section{The geometric COnstruction of the Jantzen Filtration}

In this part, we give the geometric construction of the Jantzen filtration in the affine parabolic case by generalizing the result of $[\mathrm{BB}]$.

5.1. Notation. Let $R$ be any noetherian $\mathbb{C}$-algebra. To any abelian category $\mathcal{C}$ we associate a category $\mathcal{C}_{R}$ whose objects are the pairs $\left(M, \mu_{M}\right)$ with $M$ an object of $\mathcal{C}$ and $\mu_{M}: R \rightarrow \operatorname{End}_{\mathcal{C}}(M)$ a ring homomorphism. A morphism $\left(M, \mu_{M}\right) \rightarrow\left(N, \mu_{N}\right)$ is a morphism $f: M \rightarrow N$ in $\mathcal{C}$ such that $\mu_{N}(r) \circ f=f \circ \mu_{M}(r)$ for $r \in R$. The category $\mathcal{C}_{R}$ is also abelian. We have a faithful forgetful functor

$$
\text { for }: \mathcal{C}_{R} \rightarrow \mathcal{C}, \quad\left(M, \mu_{M}\right) \rightarrow M .
$$

Any functor $F: \mathcal{C} \rightarrow \mathcal{C}^{\prime}$ gives rise to a functor

$$
F_{R}: \mathcal{C}_{R} \rightarrow \mathcal{C}_{R}^{\prime}, \quad\left(M, \mu_{M}\right) \mapsto\left(F(M), \mu_{F(M)}\right)
$$

such that $\mu_{F(M)}(r)=F\left(\mu_{M}(r)\right)$ for $r \in R$. The functor $F_{R}$ is $R$-linear. If $F$ is exact, then $F_{R}$ is also exact. We have for $\circ F_{R}=F \circ$ for. Given an inductive system of categories $\left(\mathcal{C}_{\alpha}, i_{\alpha \beta}\right)$, it yields an inductive system $\left(\left(\mathcal{C}_{\alpha}\right)_{R},\left(i_{\alpha \beta}\right)_{R}\right)$, and we have a canonical equivalence

$$
\left(2 \underset{\lim }{\longrightarrow} \mathcal{C}_{\alpha}\right)_{R}=2 \underset{\lim }{\longrightarrow}\left(\left(\mathcal{C}_{\alpha}\right)_{R}\right) .
$$


5.2. The function $f_{w}$. Let $Q^{\prime}=(Q, Q)$ be the commutator subgroup of $Q$. It acts transitively on $Y_{w}^{\dagger}$ for $w \in{ }^{Q} \mathfrak{S}$. We have the following lemma.

Lemma 5.1. For any $w \in Q_{\mathfrak{S}}$ there exists a regular function $f_{w}: \bar{Y}_{w}^{\dagger} \rightarrow \mathbb{C}$ such that $f_{w}^{-1}(0)=\bar{Y}_{w}^{\dagger}-Y_{w}^{\dagger}$ and

$$
f_{w}\left(q x h^{-1}\right)=e^{w^{-1} \omega_{0}}(h) f_{w}(x), \quad q \in Q^{\prime}, x \in Y_{w}^{\dagger}, h \in T .
$$

Proof. Let $V$ denote the simple $\mathfrak{g}$-module of highest weight $\omega_{0}$. It is integrable, hence it admits an action of $G$. Let $v_{0} \in V$ be a nonzero vector in the weight space $V_{\omega_{0}}$. It is fixed under the action of $Q^{\prime}$. So the map

$$
\varphi: G \rightarrow V, \quad g \mapsto g^{-1} v_{0}
$$

maps $Q \dot{w} B$ to $B \dot{w}^{-1} v_{0}$ for any $w \in{ }^{Q} \mathfrak{S}$. Let $V\left(w^{-1}\right)$ be the $\mathcal{U}(\mathfrak{b})$-submodule of $V$ generated by the weight space $V_{w^{-1} \omega_{0}}$. We have $B \dot{w}^{-1} v_{0} \subset V\left(w^{-1}\right)$. Recall that for $w^{\prime} \in{ }^{Q} \mathfrak{S}$ we have

$$
\begin{aligned}
w^{\prime}<w & \Longleftrightarrow\left(w^{\prime}\right)^{-1}<w^{-1} \\
& \Longleftrightarrow\left(\dot{w}^{\prime}\right)^{-1} v_{0} \in \mathfrak{n} \dot{w}^{-1} v_{0}
\end{aligned}
$$

see e.g., [Ku2, Proposition 7.1.20]. Thus, if $w^{\prime} \leqslant w$, then $\varphi\left(Q \dot{w}^{\prime} B\right) \subset V\left(w^{-1}\right)$. The $\mathbb{C}$-vector space $V\left(w^{-1}\right)$ is finite dimensional. We choose a linear form $l_{w}$ : $V\left(w^{-1}\right) \rightarrow \mathbb{C}$ such that

$$
l_{w}\left(\dot{w}^{-1} v_{0}\right) \neq 0 \quad \text { and } \quad l_{w}\left(\mathfrak{n} \dot{w}^{-1} v_{0}\right)=0 .
$$

Set $\tilde{f}_{w}=l_{w} \circ \varphi$. Then for $q \in Q^{\prime}, h \in T, u \in N$ we have

$$
\begin{aligned}
\tilde{f}_{w}\left(q \dot{w} h^{-1} u\right) & =l_{w}\left(u^{-1} h \dot{w}^{-1} v_{0}\right) \\
& =e^{w^{-1} \omega_{0}}(h) l_{w}\left(\dot{w}^{-1} v_{0}\right) \\
& =e^{w^{-1} \omega_{0}}(h) \tilde{f}_{w}\left(\dot{w}^{-1}\right) .
\end{aligned}
$$

A similar calculation together with (5.2) yields that $\tilde{f}_{w}\left(Q \dot{w}^{\prime} B\right)=0$ for $w^{\prime}<w$. Hence $\tilde{f}_{w}$ defines a regular function on $\bigsqcup_{w^{\prime} \leqslant w} Q \dot{w}^{\prime} B$ which is invariant under the right action of $N$. By consequence it induces a regular function $f_{w}$ on $\bar{Y}_{w}^{\dagger}$ which has the required properties.

Remark 5.2. (a) The function $f_{w}$ above is completely determined by its value on the point $\dot{w} N / N$, hence is unique up to scalar.

(b) The lemma implies that the embedding $j_{w}: Y_{w}^{\dagger} \rightarrow X^{\dagger}$ is affine.

(c) The function $f_{w}$ is an analogue of the function defined in BB, Lemma 3.5.1] in the finite type case. Below we will use it to define the Jantzen filtration on $\mathscr{B}_{w !}^{\lambda}$. Note that $[\mathrm{BB}]$ 's function is defined on the whole enhanced flag variety (which is a smooth scheme). Although our $f_{w}$ is only defined on the singular scheme $\bar{Y}_{w}^{\dagger}$, this does not create any problem, because the definition of the Jantzen filtration is local (see Section [5.5), and each point of $\bar{Y}_{w}^{\dagger}$ admits a neighborhood $V$ which can be embedded into a smooth scheme $U$ such that $f_{w}$ extends to $U$. The choice of such an extension will not affect the filtration; see [BB, Remark 4.2.2(iii)]. 
5.3. The $\mathscr{D}$-module $\mathscr{B}^{(n)}$. Fix $\lambda \in \Lambda$ and $w \in Q_{\mathfrak{S}}$. In the rest of Section 5, we will abbreviate

$$
j=j_{w}, \quad f=f_{w}, \quad \mathscr{B}=\mathscr{B}_{w}^{\lambda}, \quad \mathscr{B}_{!}=\mathscr{B}_{w !}^{\lambda}, \quad \text { etc. }
$$

Following $[\mathrm{BB}$ ] we introduce the deformed version of $\mathscr{B}$. Recall that $R=\mathbb{C}[[s]]$ and $\wp$ is the maximal ideal. Let $x$ denote a coordinate on $\mathbb{C}$. For each integer $n>0$ set $R^{(n)}=R\left(\wp^{n}\right)$. Consider the left $\mathscr{D}_{\mathbb{C}^{*}-\text { module }}$

$$
\mathscr{I}^{(n)}=\left(\mathscr{O}_{\mathbb{C}^{*}} \otimes R^{(n)}\right) x^{s} .
$$

It is a rank one $\mathscr{O}_{\mathbb{C}^{*}} \otimes R^{(n)}$-module generated by a global section $x^{s}$ such that the action of $\mathscr{D}_{\mathbb{C}^{*}}$ is given by $x \partial_{x}\left(x^{s}\right)=s\left(x^{s}\right)$. The restriction of $f$ yields a map $Y_{w}^{\dagger} \rightarrow \mathbb{C}^{*}$. Thus $f^{*} \mathscr{I}^{(n)}$ is a left $\mathscr{D}_{Y_{w}^{\dagger}} \otimes R^{(n)}$-module. So we get a right $\mathscr{D}_{Y_{w}^{\dagger}} \otimes R^{(n)}$ _ module

$$
\mathscr{B}^{(n)}=\mathscr{B} \otimes_{\mathscr{O}_{Y_{w}^{\dagger}}} f^{*} \mathscr{I}^{(n)} .
$$

Lemma 5.3. The right $\mathscr{D}_{Y_{w}^{\dagger}} \otimes R^{(n)}$-module $\mathscr{B}^{(n)}$ is an object of $\mathbf{M}_{h}^{\tilde{\lambda}}\left(Y_{w}\right)$.

Proof. Since $R^{(n)}$ is a $\mathbb{C}$-algebra of dimension $n$ and $\mathscr{B}$ is locally free of rank one over $\mathscr{O}_{Y_{w}^{\dagger}}$, the $\mathscr{O}_{Y_{w}^{\dagger}}$-module $\mathscr{B}^{(n)}$ is locally free of rank $n$. Hence it is a holonomic

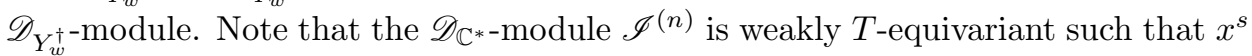
is a $T$-invariant global section. Since the map $f$ is $T$-equivariant, the $\mathscr{D}_{Y_{w}^{\dagger}}$-module $f^{*} \mathscr{I}^{(n)}$ is also weakly $T$-equivariant. Let $f^{s}$ be the global section of $f^{*} \mathscr{I}^{(n)}$ given by the image of $x^{s}$ under the inclusion

$$
\Gamma\left(\mathbb{C}^{*}, \mathscr{I}^{(n)}\right) \subset \Gamma\left(Y_{w}^{\dagger}, f^{*} \mathscr{I}^{(n)}\right) .
$$

Then $f^{s}$ is $T$-invariant. It is nowhere vanishing on $Y_{w}^{\dagger}$. Thus it yields an isomorphism of $\mathscr{O}_{Y_{w}^{\dagger}} \otimes R^{(n)}$-modules

$$
f^{*} \mathscr{I}^{(n)} \cong \mathscr{O}_{Y_{w}^{\dagger}} \otimes R^{(n)} .
$$

By consequence we have the following isomorphism

$$
\begin{aligned}
\left(\mathscr{B}^{(n)}\right)^{\dagger} & =\pi_{*}\left(\pi^{*}\left(\Omega_{Y_{w}} \otimes_{\mathscr{O}_{Y_{w}}} \mathscr{L}_{Y_{w}}^{\lambda}\right) \otimes_{\mathscr{O}_{Y_{w}^{\dagger}}} f^{*} \mathscr{I}^{(n)}\right)^{T} \\
& =\Omega_{Y_{w}} \otimes_{\mathscr{O}_{Y_{w}}} \mathscr{L}_{Y_{w}}^{\lambda} \otimes_{\mathscr{O}_{Y_{w}}} \pi_{*}\left(f^{*} \mathscr{I}^{(n)}\right)^{T} \\
& \cong \Omega_{Y_{w}} \otimes_{\mathscr{O}_{Y_{w}}} \mathscr{L}_{Y_{w}}^{\lambda} \otimes_{\mathscr{O}_{Y_{w}}}\left(\mathscr{O}_{Y_{w}} \otimes R^{(n)}\right) .
\end{aligned}
$$

See Remark 3.2 for the first equality. Next, recall from (3.5) that the right $T$-action on $Y_{w}^{\dagger}$ yields a morphism of Lie algebras

$$
\delta_{r}: \mathfrak{t} \rightarrow \Gamma\left(Y_{w}^{\dagger}, \mathscr{D}_{Y_{w}^{\dagger}}\right) .
$$

The right $\mathscr{D}_{Y_{w}}^{\dagger}$-module structure of $f^{*}\left(\mathscr{I}^{(n)}\right)$ is such that

$$
\left(f^{s} \cdot \delta_{r}(h)\right)(m)=s w^{-1} \omega_{0}(-h) f^{s}(m), \quad \forall m \in Y_{w}^{\dagger} .
$$

So the action of the element

$$
h+\lambda(h)+s w^{-1} \omega_{0}(h) \in \mathcal{U}(\mathfrak{t}) \otimes R^{(n)}
$$

on $\left(\mathscr{B}^{(n)}\right)^{\dagger}$ via the map $\delta_{r}$ vanishes. Since the multiplication by $s$ on $\left(\mathscr{B}^{(n)}\right)^{\dagger}$ is nilpotent, the action of the ideal $\mathfrak{m}_{\lambda}$ is also nilpotent. Therefore $\mathscr{B}^{(n)}$ belongs to the category $\mathbf{M}_{h}^{\tilde{\lambda}}\left(Y_{w}\right)$. 
It follows from the lemma that we have the following objects in $\mathbf{M}_{h}^{\tilde{\lambda}}(X)$

$$
\mathscr{B}_{!}^{(n)}=j_{!}\left(\mathscr{B}^{(n)}\right), \quad \mathscr{B}_{!}^{(n)}=j_{\bullet}\left(\mathscr{B}^{(n)}\right), \quad \mathscr{B}_{\bullet}^{(n)}=j \bullet\left(\mathscr{B}^{(n)}\right) .
$$

5.4. Deformed parabolic Verma modules. Fix $\lambda \in \Lambda$ and $w \in Q_{\mathfrak{S}}$ as before. Let $n>0$. If $w \cdot \lambda \in \Lambda^{+}$we will abbreviate

$$
M_{\kappa}=M_{\kappa}(w \cdot \lambda), \quad M_{\mathbf{k}}=M_{\mathbf{k}}(w \cdot \lambda), \quad M_{\mathbf{k}}^{(n)}=M_{\mathbf{k}}\left(\wp^{n}\right), \quad \mathbf{D} M_{\mathbf{k}}^{(n)}=\left(\mathbf{D} M_{\mathbf{k}}\right)\left(\wp^{n}\right) .
$$

Note that the condition is satisfied when $w$ is a shortest element in $w \mathfrak{S}(\lambda)$; see Remark 4.7

Proposition 5.4. Assume that $\lambda+\rho$ is antidominant and that $w$ is a shortest element in $w \mathfrak{S}(\lambda)$. Then there are isomorphisms of $\mathfrak{g}_{R^{(n)}}$-modules

$$
\Gamma\left(\mathscr{B}_{!}^{(n)}\right)=M_{\mathbf{k}}^{(n)}, \quad \Gamma\left(\mathscr{B}_{\bullet}^{(n)}\right)=\mathbf{D} M_{\mathbf{k}}^{(n)} .
$$

The proof will be given in Appendix B.

5.5. The geometric Jantzen filtration. Now, we define the Jantzen filtration on $\mathscr{B}$ ! following $\left[\mathrm{BB}\right.$, Sections 4.1,4.2]. Recall that $\mathscr{B}^{(n)}$ is an object of $\mathbf{M}_{h}^{\tilde{\lambda}}\left(Y_{w}\right)$. Consider the map

$$
\mu: R^{(n)} \rightarrow \operatorname{End}_{\mathbf{M}_{h}^{\tilde{\lambda}}\left(Y_{w}\right)}\left(\mathscr{B}^{(n)}\right), \quad \mu(r)(m)=r m,
$$

where $m$ denotes a local section of $\mathscr{B}^{(n)}$. Then the pair $\left(\mathscr{B}^{(n)}, \mu\right)$ is an object of the category $\mathbf{M}_{h}^{\tilde{\lambda}}\left(Y_{w}\right)_{R^{(n)}}$ defined in Section 5.1. We will abbreviate $\mathscr{B}^{(n)}=\left(\mathscr{B}^{(n)}, \mu\right)$. Fix an integer $a \geqslant 0$. Recall the morphism of functors $\psi: j_{!} \rightarrow j_{\bullet}$. We consider the morphism

$$
\psi(a, n): \mathscr{B}_{!}^{(n)} \rightarrow \mathscr{B}_{\bullet}^{(n)}
$$

in the category $\mathbf{M}_{h}^{\tilde{\lambda}}(X)_{R^{(n)}}$ given by the composition of the chain of maps

$$
\mathscr{B}_{!}^{(n)} \stackrel{j_{!}\left(\mu\left(s^{a}\right)\right)}{\longrightarrow} \mathscr{B}_{!}^{(n)} \stackrel{\psi\left(\mathscr{B}^{(n)}\right)}{\longrightarrow} \mathscr{B}_{\bullet}^{(n)} .
$$

The category $\mathbf{M}_{h}^{\tilde{\lambda}}(X)_{R^{(n)}}$ is abelian. The obvious projection $R^{(n)} \rightarrow R^{(n-1)}$ yields a canonical map

$$
\operatorname{Coker}(\psi(a, n)) \rightarrow \operatorname{Coker}(\psi(a, n-1)) .
$$

By [B, Lemma 2.1] this map is an isomorphism when $n$ is sufficiently large. We define

$$
\pi^{a}(\mathscr{B})=\operatorname{Coker}(\psi(a, n)), \quad n \gg 0 .
$$

This is an object of $\mathbf{M}_{h}^{\tilde{\lambda}}(X)_{R^{(n)}}$. We view it as an object of $\mathbf{M}_{h}^{\tilde{\lambda}}(X)$ via the forgetful functor (5.1). Now, let us consider the maps

$$
\alpha: \mathscr{B} ! \rightarrow \pi^{1}(\mathscr{B}), \quad \beta: \pi^{1}(\mathscr{B}) \rightarrow \pi^{0}(\mathscr{B})
$$

in $\mathbf{M}_{h}^{\tilde{\lambda}}(X)$ given as follows. First, since

$$
\pi^{0}(\mathscr{B})=\operatorname{Coker}\left(\psi\left(\mathscr{B}^{(n)}\right)\right) \quad \text { and } \quad \pi^{1}(\mathscr{B})=\operatorname{Coker}\left(\psi\left(\mathscr{B}^{(n)}\right) \circ j_{!}(\mu(s))\right),
$$

there is a canonical projection $\pi^{1}(\mathscr{B}) \rightarrow \pi^{0}(\mathscr{B})$. We define $\beta$ to be this map. Next, the morphism $\psi\left(\mathscr{B}^{(n)}\right)$ maps $j_{!}\left(s\left(\mathscr{B}^{(n)}\right)\right)$ to $\operatorname{Im}(\psi(1, n))$. Hence it induces a map

$$
j_{!}\left(\mathscr{B}^{(n)} / s\left(\mathscr{B}^{(n)}\right)\right) \rightarrow \pi^{1}(\mathscr{B}), \quad n \gg 0 .
$$


Composing it with the isomorphism $\mathscr{B} \cong \mathscr{B}^{(n)} / s\left(\mathscr{B}^{(n)}\right)$ we get the map $\alpha$. Let $\mu^{1}$ denote the $R^{(n)}$-action on $\pi^{1}(\mathscr{B})$. Then by [B] the sequence

$$
0 \longrightarrow \mathscr{B} ! \stackrel{\alpha}{\longrightarrow} \pi^{1}(\mathscr{B}) \stackrel{\beta}{\longrightarrow} \pi^{0}(\mathscr{B}) \longrightarrow 0,
$$

is exact and $\alpha$ induces an isomorphism

$$
\mathscr{B} ! \rightarrow \operatorname{Ker}\left(\mu^{1}(s): \pi^{1}(\mathscr{B}) \rightarrow \pi^{1}(\mathscr{B})\right) .
$$

The Jantzen filtration of $\mathscr{B}$ ! is defined by

$$
J^{i}\left(\mathscr{B}_{!}\right)=\operatorname{Ker}\left(\mu^{1}(s)\right) \cap \operatorname{Im}\left(\mu^{1}(s)^{i}\right), \quad \forall i \geqslant 0 .
$$

5.6. Comparison of the Jantzen filtrations. Fix $\lambda \in \Lambda$ and $w \in{ }^{Q} \mathfrak{S}$. Consider the Jantzen filtration $\left(J^{i} M_{\kappa}\right)$ on $M_{\kappa}$ as defined in Section 2.3 . The following proposition compares it with the geometric Jantzen filtration on $\mathscr{B}_{\text {! }}$.

Proposition 5.5. Assume that $\lambda+\rho$ is antidominant and that $w$ is a shortest element in $w \mathfrak{S}(\lambda)$. Then we have

$$
J^{i} M_{\kappa}=\Gamma\left(J^{i} \mathscr{B}_{!}\right), \quad \forall i \geqslant 0 .
$$

Proof. By Proposition 4.6(b) and Proposition 5.4 we have

$$
\Gamma\left(\mathscr{B}_{!}\right)=M_{\kappa}, \quad \Gamma\left(\mathscr{B}_{!}^{(n)}\right)=M_{\mathbf{k}}^{(n)}, \quad \Gamma\left(\mathscr{B}_{\bullet}^{(n)}\right)=\mathbf{D} M_{\mathbf{k}}^{(n)} .
$$

So the map

$$
\phi^{(n)}=\Gamma\left(\psi\left(\mathscr{B}^{(n)}\right)\right): \Gamma\left(\mathscr{B}_{!}^{(n)}\right) \rightarrow \Gamma\left(\mathscr{B}_{\bullet}^{(n)}\right) .
$$

identifies with a $\mathfrak{g}_{R^{(n)}}$-module homomorphism

$$
\phi^{(n)}: M_{\mathbf{k}}^{(n)} \rightarrow \mathbf{D} M_{\mathbf{k}}^{(n)} .
$$

Consider the projective systems $\left(M_{\mathbf{k}}^{(n)}\right),\left(\mathbf{D} M_{\mathbf{k}}^{(n)}\right), n>0$, induced by the quotient map $R^{(n)} \rightarrow R^{(n-1)}$. Their limits are respectively $M_{\mathbf{k}}$ and $\mathbf{D} M_{\mathbf{k}}$. The morphisms $\phi^{(n)}, n>0$, yield a morphism of $\mathfrak{g}_{R}$-modules

$$
\phi=\lim _{\longleftarrow} \phi^{(n)}: M_{\mathbf{k}} \rightarrow \mathbf{D} M_{\mathbf{k}}
$$

such that

$$
\phi(\wp)=\phi^{(1)}=\Gamma(\psi(\mathscr{B})) .
$$

The functor $\Gamma$ is exact by Proposition 4.1 . So the image of $\phi(\wp)$ is $\Gamma(\mathscr{B} ! \bullet)$. It is nonzero by Proposition 4.6(c). Hence $\phi$ satisfies the condition of Definition 1.6] and we have

$$
J^{i} M_{\kappa}=\left(\left\{x \in M_{\mathbf{k}} \mid \phi(x) \in s^{i} \mathbf{D} M_{\mathbf{k}}\right\}+s M_{\mathbf{k}}\right) / s M_{\mathbf{k}} .
$$

By Lemma 2.1 and Remark 1.7 the map $\phi$ is injective. So the equality above can be rewritten as

$$
J^{i} M_{\kappa}=\left(\phi\left(M_{\mathbf{k}}\right) \cap s^{i} \mathbf{D} M_{\mathbf{k}}+s \phi\left(M_{\mathbf{k}}\right)\right) / s \phi\left(M_{\mathbf{k}}\right) .
$$

Now, for $a \geqslant 0$ let

$$
\phi(a, n): M_{\mathbf{k}}^{(n)} \rightarrow \mathbf{D} M_{\mathbf{k}}^{(n)}
$$

be the $\mathfrak{g}_{R^{(n)}}$-module homomorphism given by the composition

$$
M_{\mathbf{k}}^{(n)} \stackrel{\mu\left(s^{a}\right)}{\longrightarrow} M_{\mathbf{k}}^{(n)} \stackrel{\phi^{(n)}}{\longrightarrow} \mathbf{D} M_{\mathbf{k}}^{(n)} .
$$

Then we have $\Gamma(\psi(a, n))=\phi(a, n)$. Since $\Gamma$ is exact, we have

$$
\operatorname{Coker}(\phi(a, n))=\Gamma(\operatorname{Coker}(\psi(a, n))) .
$$


So the discussion in Section [5.5 and the exactness of $\Gamma$ yields that the canonical map

$$
\operatorname{Coker}(\phi(a, n)) \rightarrow \operatorname{Coker}(\phi(a, n-1))
$$

is an isomorphism if $n$ is large enough. We deduce that

$$
\mathbf{D} M_{\mathbf{k}} / s^{a} M_{\mathbf{k}}=\operatorname{Coker}(\phi(a, n))=\Gamma\left(\pi^{a}(\mathscr{B})\right), \quad n \gg 0 ;
$$

see (5.4). The action of $\mu(s)$ on $\mathbf{D} M_{\mathbf{k}} / s^{a} \phi\left(M_{\mathbf{k}}\right)$ is nilpotent, because $\mu(s)$ is nilpotent on $\mathbf{D} M_{\mathbf{k}}^{(n)}$. Furthermore, $\Gamma$ maps the exact sequence (5.5) to an exact sequence

$$
0 \longrightarrow M_{\kappa} \longrightarrow \mathbf{D} M_{\mathbf{k}} / s \phi\left(M_{\mathbf{k}}\right) \longrightarrow \mathbf{D} M_{\mathbf{k}} / \phi\left(M_{\mathbf{k}}\right) \longrightarrow 0,
$$

and the first map yields an isomorphism

$$
M_{\kappa}=\operatorname{Ker}\left(\mu(s): \mathbf{D} M_{\mathbf{k}} / s \phi\left(M_{\mathbf{k}}\right) \rightarrow \mathbf{D} M_{\mathbf{k}} / s \phi\left(M_{\mathbf{k}}\right)\right) .
$$

Note that since $\mathbf{D} M_{\mathbf{k}}$ is a free $R$-module, for $x \in \mathbf{D} M_{\mathbf{k}}$ if $s x \in s \phi\left(M_{\mathbf{k}}\right)$, then $x \in \phi\left(M_{\mathbf{k}}\right)$. So by (5.6) and the exactness of $\Gamma$, we have for $i \geqslant 0$,

$$
\begin{aligned}
\Gamma\left(J^{i} \mathscr{B}_{!}\right) & =\operatorname{Ker}(\mu(s)) \cap \operatorname{Im}\left(\mu(s)^{i}\right) \\
& =\left(\phi\left(M_{\mathbf{k}}\right) \cap s^{i} \mathbf{D} M_{\mathbf{k}}+s \phi\left(M_{\mathbf{k}}\right)\right) / s \phi\left(M_{\mathbf{k}}\right) \\
& =J^{i} M_{\kappa} .
\end{aligned}
$$

The proposition is proved.

\section{Proof of the MAIN THEOREM}

6.1. Mixed Hodge modules. Let $Z$ be a smooth scheme. Let $\mathbf{M H M}(Z)$ be the category of mixed Hodge modules on $Z$ [Sa]. It is an abelian category. Each object $\mathscr{M}$ of $\operatorname{MHM}(Z)$ carries a canonical filtration

$$
W^{\bullet} \mathscr{M}=\cdots W^{k} \mathscr{M} \supset W^{k-1} \mathscr{M} \cdots,
$$

called the weight filtration. For each $k \in \mathbb{Z}$ the Tate twist is an auto-equivalence

$$
(k): \operatorname{MHM}(Z) \rightarrow \operatorname{MHM}(Z), \quad \mathscr{M} \mapsto \mathscr{M}(k)
$$

such that $W^{\bullet}(\mathscr{M}(k))=W^{\bullet+2 k}(\mathscr{M})$. Let $\operatorname{Perv}(Z)$ be the category of perverse sheaves on $Z$ with coefficient in $\mathbb{C}$. There is an exact forgetful functor

$$
\varrho: \operatorname{MHM}(Z) \rightarrow \operatorname{Perv}(Z) .
$$

For any locally closed affine embedding $i: Z \rightarrow Y$ we have exact functors

$$
i_{!}, i_{\bullet}: \operatorname{MHM}(Z) \rightarrow \operatorname{MHM}(Y)
$$

which correspond via $\varrho$ to the same named functors on the categories of perverse sheaves.

If $Z$ is not smooth we embed it into a smooth variety $Y$ and we define $\operatorname{MHM}(Z)$ as the full subcategory of $\operatorname{MHM}(Y)$ consisting of the objects supported on $Z$. It is independent of the choice of the embedding for the same reason as for $\mathscr{D}$-modules. 
6.2. The graded multiplicities of $\mathscr{B}_{x ! \bullet}^{\lambda}$ in $\mathscr{B}_{w !}^{\lambda}$. Now, let us calculate the multiplicities of a simple object $\mathscr{B}_{x ! \bullet}^{\lambda}$ in the successive quotients of the Jantzen filtration of $\mathscr{B}_{w !}^{\lambda}$ for $x, w \in{ }^{Q} \mathfrak{S}$ with $x \leqslant w$.

We fix once for all an element $v \in \mathfrak{S}$, and we consider the Serre subcategory $\mathbf{M}_{0}^{\lambda}\left(\bar{X}_{v}\right)$ of $\mathbf{M}_{h}^{\lambda}\left(\bar{X}_{v}\right)$ generated by the objects $\mathscr{A}_{w !}^{\lambda}$. with $w \leqslant v, w \in \mathfrak{S}$. The de Rham functor yields an exact fully faithful functor

$$
\mathrm{DR}: \mathbf{M}_{0}^{\lambda}\left(\bar{X}_{v}\right) \longrightarrow \operatorname{Perv}\left(\bar{X}_{v}\right) \text {. }
$$

See e.g., [KT1, Section 4]. Let $\mathbf{M H M}_{0}\left(\bar{X}_{v}\right)$ be the full subcategory of $\mathbf{M H M}\left(\bar{X}_{v}\right)$ consisting of objects whose image by $\varrho$ belong to the image of the functor DR. There exists a unique exact functor

$$
\eta: \mathbf{M H M}_{0}\left(\bar{X}_{v}\right) \rightarrow \mathbf{M}_{0}^{\lambda}\left(\bar{X}_{v}\right)
$$

such that DR $\circ \eta=\varrho$. An object $\mathscr{M}$ in $\operatorname{MHM}_{0}\left(\bar{X}_{v}\right)$ is pure of weight $i$ if we have $W^{k} \mathscr{M} / W^{k-1} \mathscr{M}=0$ for any $k \neq i$. For any $w \in \mathfrak{S}, w \leqslant v$, there is a unique simple object $\tilde{\mathscr{A}}_{w}^{\lambda}$ in $\mathbf{M H M}\left(X_{w}\right)$ pure of weight $l(w)$ such that $\eta\left(\tilde{\mathscr{A}}_{w}^{\lambda}\right)=\mathscr{A}_{w}^{\lambda}$; see e.g. [KT2]. Let

$$
\tilde{\mathscr{A}}_{w !}^{\lambda}=\left(i_{w}\right) !\left(\tilde{\mathscr{A}}_{w}^{\lambda}\right), \quad \tilde{\mathscr{A}}_{w ! \bullet}^{\lambda}=\left(i_{w}\right) ! \bullet\left(\tilde{\mathscr{A}}_{w}^{\lambda}\right) .
$$

They are objects of $\mathbf{M H M}_{0}\left(\bar{X}_{v}\right)$ such that

$$
\eta\left(\tilde{\mathscr{A}}_{w !}^{\lambda}\right)=\mathscr{A}_{w !}^{\lambda}, \quad \eta\left(\tilde{\mathscr{A}}_{w ! \bullet}^{\lambda}\right)=\mathscr{A}_{w ! \bullet}^{\lambda} .
$$

Now, assume that $w \in Q_{\mathfrak{S}}$ and $w \leqslant v$. Recall that $\mathscr{B}_{w}^{\lambda} \in \mathbf{M}^{\lambda}\left(Y_{w}\right)$, and that $\mathscr{B}_{w !}^{\lambda} \in \mathbf{M}^{\lambda}(X)$ can be viewed as an object of $\mathbf{M}^{\lambda}\left(\bar{X}_{v}\right)$. We define similarly the objects $\tilde{\mathscr{B}}_{w}^{\lambda} \in \mathbf{M H M}\left(Y_{w}\right)$ and $\tilde{\mathscr{B}}_{w !}^{\lambda}, \tilde{\mathscr{B}}_{w ! \bullet}^{\lambda} \in \mathbf{M H M}_{0}\left(\bar{X}_{v}\right)$ such that

$$
\eta\left(\tilde{\mathscr{B}}_{w !}^{\lambda}\right)=\mathscr{B}_{w !}^{\lambda}, \quad \eta\left(\tilde{\mathscr{B}}_{w ! \bullet}^{\lambda}\right)=\mathscr{B}_{w ! \bullet}^{\lambda} .
$$

The object $\tilde{\mathscr{B}}_{w \text { ! }}^{\lambda}$ has a canonical weight filtration $W^{\bullet}$. We set $J^{k} \mathscr{B}_{w !}^{\lambda}=\mathscr{B}_{w !}^{\lambda}$ for $k<0$. The following proposition is due to Gabber and Beilinson-Bernstein [BB, Theorem 5.1.2, Corollary 5.1.3].

Proposition 6.1. We have $\eta\left(W^{l(w)-k} \tilde{\mathscr{B}}_{w !}^{\lambda}\right)=J^{k} \mathscr{B}_{w !}^{\lambda}$ in $\mathbf{M}_{0}^{\lambda}\left(\bar{X}_{v}\right)$ for all $k \in \mathbb{Z}$.

So the problem that we posed at the beginning of the section reduces to calculate the multiplicities of $\tilde{\mathscr{B}}_{x ! \bullet}^{\lambda}$ in $\tilde{\mathscr{B}}_{w !}^{\lambda}$ in the category $\mathbf{M H M}_{0}\left(\bar{X}_{v}\right)$. Let $q$ be a formal parameter. The Hecke algebra $\mathscr{H}_{q}(\mathfrak{S})$ of $\mathfrak{S}$ is a $\mathbb{Z}\left[q, q^{-1}\right]$-algebra with a $\mathbb{Z}\left[q, q^{-1}\right]$ basis $\left\{T_{w}\right\}_{w \in \mathfrak{S}}$ whose multiplication is given by

$$
\begin{gathered}
T_{w_{1}} T_{w_{2}}=T_{w_{1} w_{2}}, \quad \text { if } l\left(w_{1} w_{2}\right)=l\left(w_{1}\right)+l\left(w_{2}\right), \\
\left(T_{s_{i}}+1\right)\left(T_{s_{i}}-q\right)=0, \quad 0 \leqslant i \leqslant m-1 .
\end{gathered}
$$

On the other hand, the Grothendieck group $\left[\mathbf{M H M}_{0}\left(\bar{X}_{v}\right)\right]$ is a $\mathbb{Z}\left[q, q^{-1}\right]$-module such that

$$
q^{k}[\mathscr{M}]=[\mathscr{M}(-k)], \quad k \in \mathbb{Z}, \mathscr{M} \in \mathbf{M H M}_{0}\left(\bar{X}_{v}\right) .
$$

For $x \in \mathfrak{S}$ with $x \leqslant v$ consider the closed embedding

$$
c_{x}: \mathrm{pt} \rightarrow \bar{X}_{v}, \quad \mathrm{pt} \mapsto \dot{x} B / B .
$$

There is an injective $\mathbb{Z}\left[q, q^{-1}\right]$-module homomorphism; see e.g., [KT2, (5.4)],

$$
\begin{aligned}
\Psi:\left[\mathbf{M H M}_{0}\left(\bar{X}_{v}\right)\right] & \longrightarrow \mathscr{H}_{q}(\mathfrak{S}), \\
{[\mathscr{M}] } & \longmapsto \sum_{x \leqslant v} \sum_{k \in \mathbb{Z}}(-1)^{k}\left[H^{k} c_{x}^{*}(\mathscr{M})\right] T_{x} .
\end{aligned}
$$


The desired multiplicities are given by the following lemma.

Lemma 6.2. For $w \in Q_{\mathfrak{S}}$ we have

$$
\Psi\left(\left[\tilde{\mathscr{B}}_{w ! \bullet}^{\lambda}\right]\right)=\sum_{x \in Q_{\mathfrak{S}, x \leqslant w}}(-1)^{l(w)-l(x)} P_{x, w} \Psi\left(\left[\tilde{\mathscr{B}}_{x !}^{\lambda}\right]\right),
$$

where $P_{x, w} \in \mathbb{Z}\left[q, q^{-1}\right]$ is the Kazhdan-Lusztig polynomial.

Proof. Since the choice for the element $v$ above is arbitrary, we may assume that $w \leqslant v$. By the definition of $\Psi$ we have

$$
\Psi\left(\left[\tilde{\mathscr{A}}_{w !}^{\lambda}\right]\right)=(-1)^{l(w)} T_{w} .
$$

By [KL1], KT1], we have

$$
\Psi\left(\left[\tilde{\mathscr{A}}_{w ! \bullet}^{\lambda}\right]\right)=(-1)^{l(w)} \sum_{x \in \mathfrak{S}} P_{x, w} T_{x} .
$$

Next, for $x \in{ }^{Q} \mathfrak{S}$ with $x \leqslant v$ we have

$$
\begin{aligned}
\Psi\left(\left[\tilde{\mathscr{B}}_{x !}^{\lambda}\right]\right) & =\sum_{y \in \mathfrak{S}_{0}} \sum_{k \in \mathbb{Z}}(-1)^{k}\left[H^{k} c_{y x}^{*}\left(\tilde{\mathscr{B}}_{x !}^{\lambda}\right)\right] T_{y x} \\
& =(-1)^{l(x)} \sum_{y \in \mathfrak{S}_{0}} T_{y x} .
\end{aligned}
$$

Since by Lemma 4.5 we have

$$
\tilde{\mathscr{A}}_{w ! \bullet}^{\lambda}=\tilde{\mathscr{B}}_{w ! \bullet}^{\lambda}
$$

the following equalities hold

$$
\begin{aligned}
\Psi\left(\left[\tilde{\mathscr{B}}_{w ! \bullet}^{\lambda}\right]\right) & =\Psi\left(\left[\tilde{\mathscr{A}}_{w ! \bullet}^{\lambda}\right]\right) \\
& =(-1)^{l(w)} \sum_{x \in Q_{\mathfrak{S}, x \leqslant w}} \sum_{y \in \mathfrak{S}_{0}} P_{y x, w} T_{y x} \\
& =(-1)^{l(w)} \sum_{x \in Q_{\mathfrak{S}, x \leqslant w}} P_{x, w} \sum_{y \in \mathfrak{S}_{0}} T_{y x} \\
& =\sum_{x \in Q_{\mathfrak{S}, x \leqslant w}}(-1)^{l(w)-l(x)} P_{x, w} \Psi\left(\left[\tilde{\mathscr{B}}_{x !}^{\lambda}\right]\right) .
\end{aligned}
$$

Here the third equality is given by the well-known identity,

$$
P_{y x, w}=P_{x, w}, \quad y \in \mathfrak{S}_{0}, x \in{ }^{Q} \mathfrak{S}, x \leqslant w .
$$

Remark 6.3. Let $x \in^{Q_{\mathfrak{S}}}$. Since $\Psi$ is injective, the equation (6.3) yields that

$$
\left[\tilde{\mathscr{B}}_{x !}^{\lambda}\right]=\sum_{y \in \mathfrak{S}_{0}}(-1)^{l(y)}\left[\tilde{\mathscr{A}}_{y x !}^{\lambda}\right]
$$

By applying the functor $\eta$ we get the following equality in $\left[\mathbf{M}_{0}^{\lambda}(X)\right]$ :

$$
\left[\mathscr{B}_{x !}^{\lambda}\right]=\sum_{y \in \mathfrak{S}_{0}}(-1)^{l(y)}\left[\mathscr{A}_{y x !}^{\lambda}\right]
$$


6.3. Proof of Theorem 0.1. Recall from (2.6) that we view $\mathcal{P}_{n}$ as a subset of $\Lambda^{+}$. By Corollary 2.14, Theorem 0.1 is a consequence of the following theorem.

Theorem 6.4. Let $\lambda, \mu$ be partitions of $n$. Then for any negative integer $\kappa$ we have

$$
d_{\lambda^{\prime} \mu^{\prime}}(q)=\sum_{i \geqslant 0}\left[J^{i} M_{\kappa}(\lambda) / J^{i+1} M_{\kappa}(\lambda): L_{\kappa}(\mu)\right] q^{i} .
$$

Here $d_{\lambda^{\prime} \mu^{\prime}}(q)$ is the polynomial defined in the introduction with $v=\exp (2 \pi i / \kappa)$.

Proof. Let $\nu \in \Lambda$ such that $\nu+\rho$ is antidominant. We may assume that $\mu, \lambda$ belong to the same orbit of $\nu$ under the dot action of $\mathfrak{S}$; see (A.7). For any $\mu \in \Lambda^{+} \cap(\mathfrak{S} \cdot \nu)$ let $w(\mu)_{\nu}$ be the shortest element in the set

$$
w(\mu)_{\nu} \mathfrak{S}(\nu)=\{w \in \mathfrak{S} \mid \mu=w \cdot \nu\} .
$$

Note that $w(\mu)_{\nu} \mathfrak{S}(\nu)$ is contained in $Q_{\mathfrak{S}}$ by Lemma 4.3 . We fix $v \in \mathfrak{S}$ such that $v \geqslant w(\gamma)_{\nu}$ for any $\gamma \in \mathcal{P}_{n}$. Let $q^{1 / 2}$ be a formal variable. We identify $q=\left(q^{1 / 2}\right)^{2}$. Let $\tilde{P}_{x, w}$ be the Kazhdan-Lusztig polynomial normalized as follows:

$$
P_{x, w}(q)=q^{(l(w)-l(x)) / 2} \tilde{P}_{x, w}\left(q^{-1 / 2}\right) .
$$

Let $\tilde{Q}_{x, w}$ be the inverse Kazhdan-Lusztig polynomial given by

$$
\sum_{x \in \mathfrak{S}} \tilde{Q}_{x, z}(-q) \tilde{P}_{x, w}(q)=\delta_{z, w}, \quad z, w \in \mathfrak{S} .
$$

Then by (6.1), (6.2) we have

$$
\left[\tilde{\mathscr{A}}_{x !}^{\nu}\right]=\sum_{w \in \mathfrak{S}} q^{(l(x)-l(w)) / 2} \tilde{Q}_{x, w}\left(q^{-1 / 2}\right)\left[\tilde{\mathscr{A}}_{w ! \bullet}^{\nu}\right], \quad \forall x \in \mathfrak{S} .
$$

By Remark 6.3 we see that

$$
\left[\tilde{\mathscr{B}}_{x !}^{\nu}\right]=\sum_{w \in Q_{\mathfrak{S}}}\left(\sum_{s \in \mathfrak{S}_{0}}(-1)^{l(s)} q^{(l(s x)-l(w)) / 2} \tilde{Q}_{s x, w}\left(q^{-1 / 2}\right)\right)\left[\tilde{\mathscr{B}}_{w ! \bullet}^{\nu}\right], \quad \forall x \in{ }^{Q_{\mathfrak{S}}} .
$$

Now, let

$$
\left[\mathbf{M}_{0}^{\nu}\left(\bar{X}_{v}\right)\right]_{q}=\left[\mathbf{M}_{0}^{\nu}\left(\bar{X}_{v}\right)\right] \otimes_{\mathbb{Z}} \mathbb{Z}\left[q^{1 / 2}, q^{-1 / 2}\right], \quad\left[\tilde{\mathcal{O}}_{\kappa}\right]_{q}=\left[\tilde{\mathcal{O}}_{\kappa}\right] \otimes_{\mathbb{Z}} \mathbb{Z}\left[q^{1 / 2}, q^{-1 / 2}\right] .
$$

We have a $\mathbb{Z}\left[q, q^{-1}\right]$-module homomorphism

$$
\begin{aligned}
\varepsilon:\left[\mathbf{M H M}_{0}\left(\bar{X}_{v}\right)\right] & \longrightarrow\left[\mathbf{M}_{0}^{\nu}\left(\bar{X}_{v}\right)\right]_{q}, \\
{[\mathscr{M}] } & \longmapsto \sum_{i \in \mathbb{Z}}\left[\eta\left(W^{i} \mathscr{M} / W^{i-1} \mathscr{M}\right)\right] q^{i / 2} .
\end{aligned}
$$

Note that $\varepsilon\left(\left[\tilde{\mathscr{B}}_{x ! \bullet}^{\nu}\right]\right)=q^{l(x) / 2}\left[\mathscr{B}_{x ! \bullet}^{\nu}\right]$ and by Proposition 6.1 we have

$$
\varepsilon\left(\left[\tilde{\mathscr{B}}_{x !}^{\nu}\right]\right)=\sum_{i \in \mathbb{N}}\left[J^{i} \mathscr{B}_{x !}^{\nu} / J^{i+1} \mathscr{B}_{x !}^{\nu}\right] q^{(l(x)-i) / 2}, \quad x \in{ }^{Q} \mathfrak{S}, x \leqslant v .
$$

Next, let

$$
\left[M_{\kappa}(\lambda)\right]_{q}=\sum_{i \in \mathbb{N}}\left[J^{i} M_{\kappa}(\lambda) / J^{i+1} M_{\kappa}(\lambda)\right] q^{-i / 2} .
$$

Then by Proposition 5.5, we have

$$
\Gamma \varepsilon\left(\left[\tilde{\mathscr{B}}_{w(\lambda)_{\nu} !}^{\nu}\right]\right)=q^{l\left(w(\lambda)_{\nu}\right) / 2}\left[M_{\kappa}(\lambda)\right]_{q} .
$$


On the other hand, by (6.6) we have

$$
\begin{aligned}
& \Gamma \varepsilon\left(\left[\tilde{\mathscr{B}}_{w(\lambda)_{\nu} !}^{\nu}\right]\right) \\
& =\sum_{w \in Q_{\mathfrak{S}}}\left(\sum_{s \in \mathfrak{S}_{0}}(-1)^{l(s)} q^{\left(l\left(s w(\lambda)_{\nu}\right)-l(w)\right) / 2} \tilde{Q}_{s w(\lambda)_{\nu}, w}\left(-q^{1 / 2}\right)\right) \Gamma \varepsilon\left[\tilde{\mathscr{B}}_{w ! \bullet}^{\nu}\right] \\
& =\sum_{\mu \in \mathcal{P}_{n}}\left(\sum_{s \in \mathfrak{S}_{0}}(-1)^{l(s)} q^{\left(l\left(s w(\lambda)_{\nu}\right)-l\left(w(\mu)_{\nu}\right)\right) / 2} \tilde{Q}_{s w(\lambda)_{\nu}, w(\mu)_{\nu}}\left(q^{-1 / 2}\right)\right) q^{l\left(w(\mu)_{\nu}\right) / 2}\left[L_{\kappa}(\mu)\right] .
\end{aligned}
$$

Here in the second equality we have used Proposition 4.6(c) and the fact that $\mathcal{P}_{n}$ is an ideal in $\Lambda^{+}$. Note that $l\left(s w(\lambda)_{\nu}\right)=l\left(w(\lambda)_{\nu}\right)-l(s)$ for $s \in \mathfrak{S}_{0}$ by Lemma 4.3(b). We deduce that

$$
\left[M_{\kappa}(\lambda)\right]_{q}=\sum_{\mu \in \mathcal{P}_{n}}\left(\sum_{s \in \mathfrak{S}_{0}}\left(-q^{-1 / 2}\right)^{l(s)} \tilde{Q}_{s w(\lambda)_{\nu}, w(\mu)_{\nu}}\left(q^{-1 / 2}\right)\right)\left[L_{\kappa}(\mu)\right]
$$

By [L, Proposition 5], we have

$$
d_{\lambda^{\prime}, \mu^{\prime}}\left(q^{-1 / 2}\right)=\sum_{s \in \mathfrak{S}_{0}}\left(-q^{-1 / 2}\right)^{l(s)} \tilde{Q}_{s w(\lambda)_{\nu}, w(\mu)_{\nu}}\left(q^{-1 / 2}\right),
$$

see also the beginning of the proof of Proposition 6 in loc. cit., and [LT2, Lemma 2.2] for instance. We deduce that

$$
\sum_{i \in \mathbb{N}}\left[J^{i} M_{\kappa}(\lambda) / J^{i+1} M_{\kappa}(\lambda)\right] q^{i}=\sum_{\mu \in \mathcal{P}_{n}} d_{\lambda^{\prime}, \mu^{\prime}}(q)\left[L_{\kappa}(\mu)\right] .
$$

The theorem is proved.

Remark 6.5. The $q$-multiplicities of the Weyl modules $W_{v}(\lambda)$ have also been considered in $[\mathrm{Ar}]$ and $[\mathrm{RT}]$. Both papers are of combinatorial nature, and are very different from the approach used here. In $\mathrm{Ar}]$ Ariki defined a grading on the $q$-Schur algebra and he proved that the $q$-multiplicities of the Weyl module with respect to this grading is also given by the same polynomials $d_{\lambda^{\prime}, \mu^{\prime}}$. However, it not clear to us how to relate this grading to the Jantzen filtration.

Remark 6.6. The radical filtration $C^{\bullet}(M)$ of an object $M$ in an abelian category $\mathcal{C}$ is given by putting $C^{0}(M)=M$ and $C^{i+1}(M)$ to be the radical of $C^{i}(M)$ for $i \leqslant 0$. It follows from [BB, Lemma 5.2.2] and Proposition 5.5 that the Jantzen filtration of $\mathscr{B}$ ! coincides with the radical filtration. If $\lambda \in \Lambda$ such that $\lambda+\rho$ is antidominant and regular, then the exact functor $\Gamma$ is faithful; see [BD, Theorem 7.15.6]. In this case, we have

$$
\Gamma\left(C^{\bullet}\left(\mathscr{B}_{!}\right)\right)=C^{\bullet}\left(\Gamma\left(\mathscr{B}_{!}\right)\right)=C^{\bullet} M_{\kappa}(\lambda) .
$$

So the Jantzen filtration on $M_{\kappa}(\lambda)$ coincides with the radical filtration. If we have further $\lambda \in \mathcal{P}_{n}$ and $\kappa \leqslant-3$, then by the equivalence in Proposition 2.13 we deduce that the Jantzen filtration of $W_{v}(\lambda)$ also coincides with the radical filtration. This is compatible with recent result of Parshall-Scott [PS], where they computed the radical filtration of $W_{v}(\lambda)$ under the same assumption of regularity here but without assuming $\kappa \leqslant-3$. We conjecture that for any $\lambda$ the Jantzen filtration on $M_{\kappa}(\lambda)$ coincides with the radical filtration.

Remark 6.7. The results of Sections 4 5, 6hold for any standard parabolic subgroup $Q$ of $G$ with the same proof. In particular, it allows us to calculate the graded decomposition matrices associated with the Jantzen filtration of the parabolic Verma modules in more general cases. 


\section{ApPendix A. Kashiwara-TANisAKi's CONSTRUCTION, TRANSLATION FUnCTORs AND Proof of Proposition 4.2}

The goal of this appendix is to prove Proposition 4.2. We first consider the case when $\lambda+\rho$ is regular. In this case, the result is essentially due to Kashiwara and Tanisaki KT1. However, the setting of loc. cit. is slightly different from the one used here. So we will first recall their construction and adapt it to our setting to complete the proof of the proposition in the regular case. Next, we give a geometric construction of the translation functor for the affine category $\mathcal{O}$ inspired from $\mathrm{BG}$, and apply it to deduce the result for singular blocks. We will use the same notation as in Section 4 .

A.1. The Kashiwara affine flag variety. Recall that $\Pi$ is the root system of $\mathfrak{g}$ and $\Pi^{+}$is the set of positive root. Write $\Pi^{-}=-\Pi^{+}$. For $\alpha \in \Pi$ we write

$$
\mathfrak{g}_{\alpha}=\{x \in \mathfrak{g} \mid[h, x]=\alpha(h) x, \quad \forall h \in \mathfrak{t}\} .
$$

For any subset $\Upsilon$ of $\Pi^{+}, \Pi^{-}$we set respectively

$$
\mathfrak{n}(\Upsilon)=\bigoplus_{\alpha \in \Upsilon} \mathfrak{g}_{\alpha}, \quad \mathfrak{n}^{-}(\Upsilon)=\bigoplus_{\alpha \in \Upsilon} \mathfrak{g}_{\alpha}
$$

For $\alpha=\sum_{i=0}^{m-1} h_{i} \alpha_{i} \in \Pi$ we write $\operatorname{ht}(\alpha)=\sum_{i=0}^{m-1} h_{i}$ and for $l \in \mathbb{N}$ we set

$$
\Pi_{l}^{-}=\left\{\alpha \in \Pi^{-} \mid \operatorname{ht}(\alpha) \leqslant-l\right\}, \quad \mathfrak{n}_{l}^{-}=\mathfrak{n}^{-}\left(\Pi_{l}^{-}\right) .
$$

Consider the group scheme $L^{-} G_{0}=G_{0}\left(\mathbb{C}\left[\left[t^{-1}\right]\right]\right)$. Let $B^{-}$be the preimage of $B_{0}^{-}$ by the map

$$
L^{-} G_{0} \rightarrow G_{0}, \quad t^{-1} \mapsto 0,
$$

where $B_{0}^{-}$is the Borel subgroup of $G_{0}$ opposite to $B_{0}$. Let $N^{-}$be the prounipotent radical of $B^{-}$. Let $N_{l}^{-} \subset B^{-}$be the group subscheme given by

$$
N_{l}^{-}={\underset{l}{k}}_{\lim } \exp \left(\mathfrak{n}_{l}^{-} / \mathfrak{n}_{k}^{-}\right) \text {. }
$$

Let $\mathfrak{X}$ be the Kashiwara affine flag variety; see $[\mathrm{K}$. It is a quotient scheme $\mathfrak{X}=G_{\infty} / B$, where $G_{\infty}$ is a coherent scheme with a locally free left action of $B^{-}$and a locally free right action of $B$. The scheme $\mathfrak{X}$ is coherent, prosmooth, nonquasi-compact, locally of countable type, with a left action of $B^{-}$. There is a right $T$-torsor

$$
\pi: \mathfrak{X}^{\dagger}=G_{\infty} / N \rightarrow \mathfrak{X} .
$$

For any subscheme $Z$ of $\mathfrak{X}$ let $Z^{\dagger}$ be its preimage by $\pi$. Let

$$
\mathfrak{X}=\bigsqcup_{w \in \mathfrak{S}} \stackrel{\circ}{\mathfrak{X}}^{w} .
$$

be the $B^{-}$-orbit decomposition. Then $\mathfrak{X}$ is covered by the following open sets

$$
\mathfrak{X}^{w}=\bigsqcup_{v \leqslant w} \stackrel{\circ}{\mathfrak{X}}^{v} .
$$

For each $w$ there is a canonical closed embedding $\bar{X}_{w} \rightarrow \mathfrak{X}^{w}$. Moreover, for any integer $l$ that is large enough, the group $N_{l}^{-}$acts locally freely on $\mathfrak{X}^{w}, \mathfrak{X}^{w \dagger}$, the quotients

$$
\mathfrak{X}_{l}^{w}=N_{l}^{-} \backslash \mathfrak{X}^{w}, \quad \mathfrak{X}_{l}^{w \dagger}=N_{l}^{-} \backslash \mathfrak{X}^{w \dagger}
$$


are smooth schemes 3 , and the induced morphism

$$
\bar{X}_{w} \rightarrow \mathfrak{X}_{l}^{w}
$$

is a closed immersion. See [KT1, Lemma 2.2.1]. Furthermore, we have

$$
\bar{X}_{w}^{\dagger}=\bar{X}_{w} \times_{\mathfrak{X}_{l}^{w}} \mathfrak{X}_{l}^{w \dagger}
$$

In particular, we get a closed embedding of $\bar{X}_{w}^{\dagger} \rightarrow \bar{X}_{w}$ into the $T$-torsor $\mathfrak{X}_{l}^{w \dagger} \rightarrow \mathfrak{X}_{l}^{w}$. This implies that the $T$-torsor $\pi: X^{\dagger} \rightarrow X$ is admissible. Finally, let

$$
p_{l_{1} l_{2}}: \mathfrak{X}_{l_{1}}^{w \dagger} \rightarrow \mathfrak{X}_{l_{2}}^{w \dagger}, \quad p_{l}: \mathfrak{X}^{w \dagger} \rightarrow \mathfrak{X}_{l}^{w \dagger}, \quad l_{1} \geqslant l_{2}
$$

be the canonical projections. They are affine morphisms.

A.2. The category $\mathbf{H}^{\tilde{\lambda}}(X)$. Fix $w, y \in \mathfrak{S}$ with $y \geqslant w$. For $l_{1} \geqslant l_{2}$ large enough, the functor

$$
\left(p_{l_{1} l_{2}}\right) \bullet: \mathbf{M}_{h}^{\tilde{\lambda}}\left(\mathfrak{X}_{l_{1}}^{y}, \bar{X}_{w}\right) \rightarrow \mathbf{M}_{h}^{\tilde{\lambda}}\left(\mathfrak{X}_{l_{2}}^{y}, \bar{X}_{w}\right)
$$

yields a filtering projective system of categories, and we set

$$
\mathbf{H}^{\tilde{\lambda}}\left(\mathfrak{X}^{y}, \bar{X}_{w}\right)=\underset{\longleftarrow_{l}}{2 \lim _{h}} \mathbf{M}_{h}^{\tilde{\lambda}}\left(\mathfrak{X}_{l}^{y}, \bar{X}_{w}\right) .
$$

For $z \geqslant y$ let $j_{y z}: \mathfrak{X}^{y \dagger} \rightarrow \mathfrak{X}^{z \dagger}$ be the canonical open embedding. It yields a map $j_{y z}: \mathfrak{X}_{l}^{y \dagger} \rightarrow \mathfrak{X}_{l}^{z \dagger}$ for each $l$. The pull-back functors by these maps yield, by base change, a morphism of projective systems of categories

$$
\left(\mathbf{M}_{h}^{\tilde{\lambda}}\left(\mathfrak{X}_{l}^{z}, \bar{X}_{w}\right)\right)_{l} \rightarrow\left(\mathbf{M}_{h}^{\tilde{\lambda}}\left(\mathfrak{X}_{l}^{y}, \bar{X}_{w}\right)\right)_{l} .
$$

Hence we get a map

$$
\mathbf{H}^{\tilde{\lambda}}\left(\mathfrak{X}^{z}, \bar{X}_{w}\right) \rightarrow \mathbf{H}^{\tilde{\lambda}}\left(\mathfrak{X}^{y}, \bar{X}_{w}\right) .
$$

As $y, z$ varies these maps yield again a projective system of categories and we set

$$
\mathbf{H}^{\tilde{\lambda}}\left(\bar{X}_{w}\right)=2 \lim _{y \geqslant w} \mathbf{H}^{\tilde{\lambda}}\left(\mathfrak{X}^{y}, \bar{X}_{w}\right) .
$$

Finally, for $w \leqslant v$ the category $\mathbf{H}^{\tilde{\lambda}}\left(\bar{X}_{w}\right)$ is canonically a full subcategory of $\mathbf{H}^{\tilde{\lambda}}\left(\bar{X}_{v}\right)$. We define

$$
\mathbf{H}^{\tilde{\lambda}}(X)=\underset{w}{2 \lim _{w}} \mathbf{H}^{\tilde{\lambda}}\left(\bar{X}_{w}\right) .
$$

This definition is inspired from [KT1, where the authors considered the categories $\mathbf{M}_{h}^{\lambda}\left(\mathfrak{X}_{l}^{y}, \bar{X}_{w}\right)$ instead of the categories $\mathbf{M}_{h}^{\tilde{\lambda}}\left(\mathfrak{X}_{l}^{y}, \bar{X}_{w}\right)$. Finally, note that since the category $\mathbf{H}^{\tilde{\lambda}}\left(\bar{X}_{w}\right)$ is equivalent to $\mathbf{M}_{h}^{\tilde{\lambda}}\left(\mathfrak{X}_{l}^{y}, \bar{X}_{w}\right)$ for $y, l$ large enough, and since the latter is equivalent to $\mathbf{M}_{h}^{\tilde{\lambda}}\left(\bar{X}_{w}\right)$ (see Section [3.1), we have an equivalence of categories

$$
\mathbf{H}^{\tilde{\lambda}}(X) \cong \mathbf{M}_{h}^{\tilde{\lambda}}(X)
$$

\footnotetext{
${ }^{3}$ For $l$ large enough the scheme $\mathfrak{X}_{l}^{w}$ is separated (hence quasi-separated). To see this, one uses the fact that $\mathfrak{X}^{w}$ is separated and applies [TT, Proposition C.7].
} 
A.3. The functors $\hat{\Gamma}$ and $\bar{\Gamma}$. For an object $\mathscr{M}$ of $\mathbf{H}^{\tilde{\lambda}}(X)$, there exists $w \in \mathfrak{S}$ such that $\mathscr{M}$ is an object of the subcategory $\mathbf{H}^{\tilde{\lambda}}\left(\bar{X}_{w}\right)$. Thus $\mathscr{M}$ is represented by a system $\left(\mathscr{M}_{l}^{y}\right)_{y \geqslant w, l}$, with $\mathscr{M}_{l}^{y} \in \mathbf{M}_{h}^{\tilde{\lambda}}\left(\mathfrak{X}_{l}^{y}, \bar{X}_{w}\right)$ and $l$ large enough. For $l_{1} \geqslant l_{2}$ there is a canonical map

$$
\left(p_{l_{1} l_{2}}\right)_{*}\left(\mathscr{M}_{l_{1}}^{y}\right) \rightarrow\left(p_{l_{1} l_{2}}\right) \bullet\left(\mathscr{M}_{l_{1}}^{y}\right)=\mathscr{M}_{l_{2}}^{y} .
$$

It yields a map (see (3.6) for the notation)

$$
\Gamma\left(\mathfrak{X}_{l_{1}}^{y}, \mathscr{M}_{l_{1}}^{y}\right) \rightarrow \Gamma\left(\mathfrak{X}_{l_{2}}^{y}, \mathscr{M}_{l_{2}}^{y}\right) .
$$

Next, for $y, z \geqslant w$ and $l$ large enough, we have a canonical isomorphism

$$
\Gamma\left(\mathfrak{X}_{l}^{y}, \mathscr{M}_{l}^{y}\right)=\Gamma\left(\mathfrak{X}_{l}^{z}, \mathscr{M}_{l}^{z}\right) .
$$

Following [KT1, we choose a $y \geqslant w$ and we set

$$
\hat{\Gamma}(\mathscr{M})=\lim _{l} \Gamma\left(\mathfrak{X}_{l}^{y}, \mathscr{M}_{l}^{y}\right) .
$$

This definition does not depend on the choice of $w, y$. Now, regard $\mathscr{M}$ as an object of $\mathbf{M}_{h}^{\tilde{\lambda}}(X)$. Recall the object $\mathscr{M}^{\dagger} \in \mathbf{O}(X)$ from Section 3.4. Suppose that $\mathscr{M}^{\dagger}$ is represented by a system $\left(\mathscr{M}_{y}^{\mathscr{O}}\right)_{y \geqslant w}$ with $\mathscr{M}_{y}^{\mathscr{O}} \in \mathbf{O}\left(\bar{X}_{y}\right)$. By definition we have $\mathscr{M}_{y}^{\mathscr{O}}=\left(i^{!} \mathscr{M}_{l}^{y}\right)^{\dagger}$, where $i$ denotes the closed embedding $\bar{X}_{y}^{\dagger} \rightarrow \mathfrak{X}_{l}^{y \dagger}$; see (A.1). Therefore we have

$$
\begin{aligned}
\Gamma\left(\bar{X}_{y}, \mathscr{M}_{y}^{\mathscr{O}}\right) & =\Gamma\left(\bar{X}_{y}, i^{!}\left(\mathscr{M}_{l}^{y}\right)^{\dagger}\right) \\
& \subset \Gamma\left(\mathfrak{X}_{l}^{y}, \mathscr{M}_{l}^{y}\right) .
\end{aligned}
$$

Next, recall that we have

$$
\Gamma(\mathscr{M})=\Gamma\left(X, \mathscr{M}^{\dagger}\right)=\underset{y}{\lim } \Gamma\left(\bar{X}_{y}, \mathscr{M}_{y}^{\mathscr{O}}\right) .
$$

So by first taking the projective limit on the right hand side of (A.2) with respect to $l$ and then taking the inductive limit on the left hand side with respect to $y$ we get an inclusion

$$
\Gamma(\mathscr{M}) \subset \hat{\Gamma}(\mathscr{M}) .
$$

It identifies $\Gamma(\mathscr{M})$ with the subset of $\hat{\Gamma}(\mathscr{M})$ consisting of the sections supported on subschemes (of finite type) of $X$.

The vector space $\hat{\Gamma}(\mathscr{M})$ has a $\mathfrak{g}$-action; see [KT1, Section 2.3]. The vector space $\Gamma(\mathscr{M})$ has also a $\mathfrak{g}$-action by Section 4.3 . The inclusion is compatible with these $\mathfrak{g}$-actions. Following loc. cit., let

$$
\bar{\Gamma}(\mathscr{M}) \subset \hat{\Gamma}(\mathscr{M})
$$

be the set of $\mathfrak{t}$-finite elements. It is a $\mathfrak{g}$-submodule of $\hat{\Gamma}(\mathscr{M})$.

A.4. The regular case. In this subsection we prove Proposition 4.2 in the regular case. More precisely, we prove the following result.

Proposition A.1. Let $\lambda \in \Lambda$ be such that $\lambda+\rho$ is antidominant and regular. Then we have isomorphisms of $\mathfrak{g}$-modules

(A.3) $\Gamma\left(\mathscr{A}_{v !}^{\lambda}\right)=N_{\kappa}(v \cdot \lambda), \quad \Gamma\left(\mathscr{A}_{v \bullet}^{\lambda}\right)=\mathbf{D} N_{\kappa}(v \cdot \lambda), \quad \Gamma\left(\mathscr{A}_{v ! \bullet}^{\lambda}\right)=L_{\kappa}(v \cdot \lambda), \quad \forall v \in \mathfrak{S}$. 
Proof. By [KT1, Theorem 3.4.1] under the assumption of the proposition we have isomorphisms of $\mathfrak{g}$-modules.

$\bar{\Gamma}\left(\mathscr{A}_{v !}^{\lambda}\right)=N_{\kappa}(v \cdot \lambda), \quad \bar{\Gamma}\left(\mathscr{A}_{v \bullet}^{\lambda}\right)=\mathbf{D} N_{\kappa}(v \cdot \lambda), \quad \bar{\Gamma}\left(\mathscr{A}_{v ! \bullet}^{\lambda}\right)=L_{\kappa}(v \cdot \lambda), \quad \forall v \in \mathfrak{S}$.

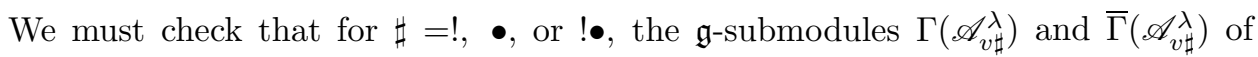
$\hat{\Gamma}\left(\mathscr{A}_{v \sharp}^{\lambda}\right)$ are equal. Let us first prove this for $\sharp=\bullet$. We will do this in several steps.

Step 1. Following [KT1] we first define a particular section $\vartheta$ in $\hat{\Gamma}\left(\mathscr{A}_{v \bullet}^{\lambda}\right)$. Let $\omega$ be a nowhere vanishing section of $\Omega_{X_{v}}$. It is unique up to a nonzero scalar. Let $t^{\lambda}$ be the nowhere vanishing section of $\mathscr{L}_{X_{v}}^{\lambda}$ such that $t^{\lambda}(u \dot{v} b)=e^{-\lambda}(b)$ for $u \in N$, $b \in B$. Then $\omega \otimes t^{\lambda}$ is a nowhere vanishing section of $\mathscr{A}_{v}^{\lambda, \dagger}$ over $X_{v}$. Now, for $y \geqslant v$ and $l$ large enough, let $i_{l}^{v}: X_{v} \rightarrow \mathfrak{X}_{l}^{y}$ be the composition of the locally closed embedding $X_{v} \rightarrow \bar{X}_{y}$ and the closed embedding $\bar{X}_{y} \rightarrow \mathfrak{X}_{l}^{y}$ in (A.1). We will denote the corresponding embedding $X_{v}^{\dagger} \rightarrow \mathfrak{X}_{l}^{y \dagger}$ again by $i_{l}^{v}$. Note that $\left(i_{l \bullet}^{v}\left(\mathscr{A}_{v}^{\lambda}\right)\right)_{l}$ represents the object $\mathscr{A}_{v \bullet}^{\lambda}$ in $\mathbf{H}^{\tilde{\lambda}}(X)$. Therefore we have

$$
\hat{\Gamma}\left(\mathscr{A}_{v \bullet}^{\lambda}\right)=\lim _{l} \Gamma\left(\mathfrak{X}_{l}^{y}, i_{l \bullet}^{v}\left(\mathscr{A}_{v}^{\lambda}\right)\right) \text {. }
$$

Consider the canonical inclusion of $\mathscr{O}_{\mathfrak{X}_{l}^{y} \text {-modules }}$

$$
i_{l *}^{v}\left(\mathscr{A}_{v}^{\lambda}\right)^{\dagger} \longrightarrow i_{l \bullet}^{v}\left(\mathscr{A}_{v}^{\lambda}\right)^{\dagger} \text {. }
$$

Let $\vartheta_{l} \in \Gamma\left(\mathfrak{X}_{l}^{y}, i_{l \bullet}^{v}\left(\mathscr{A}_{v}^{\lambda}\right)^{\dagger}\right)$ be the image of $\omega \otimes t^{\lambda}$ under this map. The family $\left(\vartheta_{l}\right)$ defines an element

$$
\vartheta \in \hat{\Gamma}\left(\mathscr{A}_{v \bullet}^{\lambda}\right)
$$

Step 2. Let $V^{y}=y B^{-} \cdot B / B$. It is an affine open set in $\mathfrak{X}^{y}$. For $l$ large enough, let $V_{l}^{y}$ be the image of $V^{y}$ in $\mathfrak{X}_{l}^{y}$ via the canonical projection $\mathfrak{X}^{y} \rightarrow \mathfrak{X}_{l}^{y}$. Write $j_{l}^{y}: V_{l}^{y} \rightarrow \mathfrak{X}_{l}^{y}$ for the inclusion. Note that $V_{l}^{y} \cong N^{-} / N_{l}^{-}$as affine spaces. Therefore, if $l$ is large enough such that $\Pi_{l}^{-} \subset \Pi^{-} \cap v \Pi^{-}$, then the right $\mathscr{D}_{X_{v}}^{\dagger}$-module structure on $\mathscr{A}_{v}^{\lambda, \dagger}$ yields an isomorphism of sheaves of $\mathbb{C}$-vector spaces over $V_{l}^{y}$,

$$
\begin{aligned}
j_{l}^{y *}\left(i_{l *}^{v} \mathscr{O}_{X_{v}}\right) \otimes \mathcal{U}\left(\mathfrak{n}^{-}\left(\Pi^{-} \cap v\left(\Pi^{-}\right)\right) / \mathfrak{n}_{l}^{-}\right) & \stackrel{\sim}{\rightarrow} j_{l}^{y *}\left(i_{l \bullet}^{v}\left(\mathscr{A}_{v}^{\lambda}\right)^{\dagger}\right), \\
f \otimes p & \mapsto(\vartheta \cdot f) \cdot \delta_{l}(p) .
\end{aligned}
$$

This yields an isomorphism of $\mathfrak{t}$-modules

$$
\Gamma\left(\mathfrak{X}_{l}^{y}, i_{l \bullet}^{v}\left(\mathscr{A}_{v}^{\lambda}\right)\right)=\mathcal{U}\left(\mathfrak{n}^{-} / \mathfrak{n}_{l}^{-}\right) \otimes \mathbb{C}_{v \cdot \lambda}
$$

see [KT1, Lemma 3.2.1]. By consequence we have an isomorphism of $\mathfrak{t}$-modules

$$
\hat{\Gamma}\left(\mathscr{A}_{v \bullet}^{\lambda}\right)=\left(\lim _{l} \mathcal{U}\left(\mathfrak{n}^{-} / \mathfrak{n}_{l}^{-}\right)\right) \otimes \mathbb{C}_{v \cdot \lambda} .
$$

Step 3. Now, let us prove $\Gamma\left(\mathscr{A}_{v \bullet}^{\lambda}\right)=\bar{\Gamma}\left(\mathscr{A}_{v \bullet}^{\lambda}\right)$. First, by (A.4) the space $\Gamma\left(\mathfrak{X}_{l}^{y}, i_{l \bullet}^{v}\left(\mathscr{A}_{v}^{\lambda}\right)\right)$ is t-locally finite. So (A.2) implies that $\Gamma\left(\mathscr{A}_{v \bullet}^{\lambda}\right)$ is the inductive limit of a system of $\mathfrak{t}$-locally finite submodules. Therefore it is itself $\mathfrak{t}$-locally finite. Hence we have

$$
\Gamma\left(\mathscr{A}_{v \bullet}^{\lambda}\right) \subset \bar{\Gamma}\left(\mathscr{A}_{v \bullet}^{\lambda}\right)
$$

To see that this is indeed an equality, note that if $m \in \hat{\Gamma}\left(\mathscr{A}_{v \bullet}^{\lambda}\right)$ is not $\mathfrak{t}$-locally finite, then by (A.5) the section $m$ is represented by an element in

$$
\lim _{l} \mathcal{U}\left(\mathfrak{n}^{-} / \mathfrak{n}_{l}^{-}\right) \otimes \mathbb{C}_{v \cdot \lambda}
$$


which does not come from $\mathcal{U}\left(\mathfrak{n}^{-}\right) \otimes \mathbb{C}_{v \cdot \lambda}$ via the obvious map. Then one sees that $m$ cannot be supported on a finite dimensional scheme, i.e., it cannot belong to $\Gamma\left(\mathscr{A}_{v \bullet}^{\lambda}\right)$. This proves that

$$
\Gamma\left(\mathscr{A}_{v \bullet}^{\lambda}\right)=\bar{\Gamma}\left(\mathscr{A}_{v \bullet}^{\lambda}\right) .
$$

Now, we can prove the other two equalities in the proposition. Since $\lambda+\rho$ is antidominant, by Proposition 4.1(a) the functor $\Gamma$ is exact on $\mathbf{M}^{\lambda}(X)$. So $\Gamma\left(\mathscr{A}_{v ! \bullet}^{\lambda}\right)$ is a $\mathfrak{g}$-submodule of $\Gamma\left(\mathscr{A}_{v \bullet}^{\lambda}\right)$. Therefore all the elements in $\Gamma\left(\mathscr{A}_{v ! \bullet}^{\lambda}\right)$ are $\mathfrak{t}$-finite, i.e., we have

$$
\Gamma\left(\mathscr{A}_{v ! \bullet}^{\lambda}\right) \subset \bar{\Gamma}\left(\mathscr{A}_{v ! \bullet}^{\lambda}\right)
$$

On the other hand, by [KT1, Theorem 3.4.1] we have

$$
\bar{\Gamma}\left(\mathscr{A}_{v ! \bullet}^{\lambda}\right) \subset \bar{\Gamma}\left(\mathscr{A}_{v \bullet}^{\lambda}\right) \text {. }
$$

Therefore, Step 3 yields that each section in $\bar{\Gamma}\left(\mathscr{A}_{v ! \bullet}^{\lambda}\right)$ is supported on a finite dimensional scheme, and hence belongs to $\Gamma\left(\mathscr{A}_{v ! \bullet}^{\lambda}\right)$. We deduce that

$$
\Gamma\left(\mathscr{A}_{v ! \bullet}^{\lambda}\right)=\bar{\Gamma}\left(\mathscr{A}_{v ! \bullet}^{\lambda}\right)
$$

Finally, since $\mathscr{A}_{v !}^{\lambda}$ has a finite composition series whose constituents are given by $\mathscr{A}_{w ! \bullet}^{\lambda}$ for $w \leqslant v$. Since both $\Gamma$ and $\bar{\Gamma}$ are exact functors on $\mathbf{M}_{0}^{\lambda}(X)$; see Proposition 4.1 and [KT1, Corollary 3.3.3, Theorem 3.4.1]. We deduce from (A.6) that $\Gamma\left(\mathscr{A}_{v !}^{\lambda}\right)$ is t-locally finite, and the sections of $\bar{\Gamma}\left(\mathscr{A}_{v !}^{\lambda}\right)$ are supported on finite dimensional subschemes. Therefore we have

$$
\Gamma\left(\mathscr{A}_{v !}^{\lambda}\right)=\bar{\Gamma}\left(\mathscr{A}_{v !}^{\lambda}\right)
$$

The proposition is proved.

A.5. Translation functors. In order to compute the images of $\mathscr{A}_{v !}^{\lambda}$ and $\mathscr{A}_{v \bullet}^{\lambda}$ in the case when $\lambda+\rho$ is not regular, we need the translation functors. For $\lambda \in \kappa^{\mathrm{t}}{ }^{*}$ such that $\lambda+\rho$ is anti-dominant, we define $\tilde{\mathcal{O}}_{\kappa, \lambda}$ to be the Serre subcategory of $\tilde{\mathcal{O}}_{\kappa}$ generated by $L_{\kappa}(w \cdot \lambda)$ for all $w \in \mathfrak{S}$. The same argument as in the proof of DGK, Theorem 4.2] yields that each $M \in \tilde{\mathcal{O}}_{\kappa}$ admits a decomposition

$$
M=\bigoplus M^{\lambda}, \quad M^{\lambda} \in \tilde{\mathcal{O}}_{\kappa, \lambda},
$$

where $\lambda$ runs over all the weights in ${ }_{\kappa} t^{*}$ such that $\lambda+\rho$ is antidominant. The projection

$$
\operatorname{pr}_{\lambda}: \tilde{\mathcal{O}}_{\kappa} \rightarrow \tilde{\mathcal{O}}_{\kappa, \lambda}, \quad M \mapsto M^{\lambda}
$$

is an exact functor. Fix two integral weights $\lambda, \mu$ in $\mathfrak{t}^{*}$ such that $\lambda+\rho, \mu+\rho$ are antidominant and the integral weight $\nu=\lambda-\mu$ is dominant. Assume that $\lambda \in{ }_{\kappa} \mathrm{t}^{*}$, then $\mu$ belongs to $\kappa^{\prime} \mathrm{t}^{*}$ for an integer $\kappa^{\prime}<\kappa$. Let $V(\nu)$ be the simple $\mathfrak{g}$-module of highest weight $\nu$. Then for any $M \in \tilde{\mathcal{O}}_{\kappa^{\prime}}$ the module $M \otimes V(\nu)$ belongs to $\tilde{\mathcal{O}}_{\kappa}$. Therefore we can define the following translation functor:

$$
\theta^{\nu}: \tilde{\mathcal{O}}_{\kappa^{\prime}, \mu} \rightarrow \tilde{\mathcal{O}}_{\kappa, \lambda}, \quad M \mapsto \operatorname{pr}_{\lambda}(M \otimes V(\nu)) ;
$$

see $\mathrm{Ku} 1$. Note that the subcategory $\tilde{\mathcal{O}}_{\kappa, \lambda}$ of $\tilde{\mathcal{O}}$ is stable under the duality $\mathbf{D}$, because $\mathbf{D}$ fixes simple modules. We have a canonical isomorphism of functors

$$
\theta^{\nu} \circ \mathbf{D}=\mathbf{D} \circ \theta^{\nu} .
$$

Indeed, it follows from (4.5) that $\mathbf{D}(M \otimes V(\nu))=\mathbf{D}(M) \otimes \mathbf{D}(V(\nu))$ as $\mathfrak{g}$-modules. Since $V(\nu)$ is simple, we have $\mathbf{D} V(\nu)=V(\nu)$. The equality A.8 follows. 
On the geometric side, recall the $T$-torsor $\pi: X^{\dagger} \rightarrow X$. For any integral weight $\lambda \in \mathfrak{t}^{*}$ the family of line bundles $\mathscr{L}_{\bar{X}}^{\lambda}$ (see Section 3.5) with $w \in \mathfrak{S}$ form a projective system of $\mathscr{O}$-modules under restriction, yielding a flat object $\mathscr{L}^{\lambda}$ of $\hat{\mathbf{O}}(X)$. Note that $\pi^{*}\left(\mathscr{L}^{\lambda}\right)$ is a line bundle on $X^{\dagger}$. For integral weights $\lambda, \mu$ in $\mathfrak{t}^{*}$ the translation functor

$$
\Theta^{\lambda-\mu}: \mathbf{M}_{0}^{\mu}(X) \rightarrow \mathbf{M}_{0}^{\lambda}(X), \quad \mathscr{M} \mapsto \mathscr{M} \otimes_{\mathscr{O}_{X^{\dagger}}} \pi^{*}\left(\mathscr{L}^{\lambda-\mu}\right),
$$

is an equivalence of categories. A quasi-inverse is given by $\Theta^{\mu-\lambda}$. By the projection formula we have

$$
\Theta^{\lambda-\mu}\left(\mathcal{A}_{w \sharp}^{\mu}\right)=\mathcal{A}_{w \sharp}^{\lambda}, \quad \text { for } \sharp=!, ! \bullet, \bullet .
$$

Now, assume that $\mu+\rho$ is antidominant. Consider the exact functor

$$
\Gamma: \mathbf{M}^{\mu}(X) \rightarrow \mathbf{M}(\mathfrak{g}), \quad \mathscr{M} \mapsto \Gamma(\mathscr{M})
$$

as in Proposition 4.1. Note that if $\mu+\rho$ is regular, then $\Gamma$ maps $\mathscr{A}_{v ! \bullet}^{\mu}$ to $L_{\kappa}(v \cdot \mu)$ by Proposition A.1. Since the subcategory $\tilde{\mathcal{O}}_{\kappa}$ of $\mathbf{M}(\mathfrak{g})$ is stable under extension, the exact functor $\Gamma$ restricts to a functor

$$
\Gamma: \mathbf{M}_{0}^{\mu}(X) \rightarrow \tilde{\mathcal{O}}_{\kappa, \mu} .
$$

The next proposition is an affine analogue of [BG, Proposition 2.8].

Proposition A.2. Let $\lambda, \mu$ be integral weights in $\mathfrak{t}^{*}$ such that $\lambda+\rho, \mu+\rho$ are antidominant and $\nu=\lambda-\mu$ is dominant. Assume further that $\mu+\rho$ is regular. Then the functors

$$
\theta^{\nu} \circ \Gamma: \mathbf{M}_{0}^{\mu}(X) \rightarrow \tilde{\mathcal{O}}_{\kappa, \lambda} \subset \mathbf{M}(\mathfrak{g}) \quad \text { and } \quad \Gamma \circ \Theta^{\nu}: \mathbf{M}_{0}^{\mu}(X) \rightarrow \mathbf{M}(\mathfrak{g})
$$

are isomorphic.

Proof. We will prove the proposition in several steps.

Step 1. First, we define a category $\mathbf{S h}(X)$ of sheaves of $\mathbb{C}$-vector spaces on $X$ and we consider $\mathfrak{g}$-modules in this category. To do this, for $w \in \mathfrak{S}$ let $\operatorname{Sh}\left(\bar{X}_{w}\right)$ be the category of sheaves of $\mathbb{C}$-vector spaces on $\bar{X}_{w}$. For $w \leqslant x$ we have a closed embedding $i_{w, x}: \bar{X}_{w} \rightarrow \bar{X}_{x}$, and an exact functor

$$
i_{w, x}^{!}: \mathbf{S h}\left(\bar{X}_{x}\right) \rightarrow \mathbf{S h}\left(\bar{X}_{w}\right), \quad \mathscr{F} \mapsto i_{w, x}^{!}(\mathscr{F}),
$$

where $i_{w, x}^{!}(\mathscr{F})$ is the subsheaf of $\mathscr{F}$ consisting of the local sections supported settheoretically on $\bar{X}_{w}$. We get a projective system of categories

$$
\left(\mathbf{S h}\left(\bar{X}_{w}\right), i_{w, x}^{!}\right)
$$

Following [BD, 7.15.10] we define the category of sheaves of $\mathbb{C}$-vector spaces on $X$ to be the projective limit

$$
\mathbf{S h}(X)=2 \lim _{\leftarrow} \mathbf{S h}\left(\bar{X}_{w}\right)
$$

This is an abelian category. By the same arguments as in the second paragraph of Section 3.7, the category $\operatorname{Sh}\left(\bar{X}_{w}\right)$ is canonically identified with a full subcategory of $\mathbf{S h}(X)$, and each object $\mathscr{F} \in \mathbf{S h}(X)$ is a direct limit

$$
\mathscr{F}=\lim _{\longrightarrow} \mathscr{F}_{w}, \quad \mathscr{F}_{w} \in \mathbf{S h}\left(\bar{X}_{w}\right) .
$$

The space of global sections of an object of $\mathbf{S h}(X)$ is given by

$$
\Gamma(X, \mathscr{F})=\lim _{\longrightarrow} \Gamma\left(\bar{X}_{w}, \mathscr{F}_{w}\right) .
$$


Next, consider the forgetful functor

$$
\mathbf{O}\left(\bar{X}_{w}\right) \rightarrow \operatorname{Sh}\left(\bar{X}_{w}\right), \quad \mathscr{N} \mapsto \mathscr{N}^{\mathbb{C}} .
$$

Recall that for $\mathscr{M} \in \mathbf{O}(X)$ we have $\mathscr{M}=\underline{\lim } \mathscr{M}_{w}$ with $\mathscr{M}_{w} \in \mathbf{O}\left(\bar{X}_{w}\right)$. The tuple of sheaves of $\mathbb{C}$-vector spaces

$$
\underset{x \geqslant w}{\lim _{x, x}} i_{w}^{!}\left(\mathscr{M}_{x}^{\mathbb{C}}\right), w \in \mathfrak{S},
$$

gives an object in $\mathbf{S h}(X)$. Let us denote it by $\mathscr{M}^{\mathbb{C}}$. The assignment $\mathscr{M} \mapsto \mathscr{M}^{\mathbb{C}}$ yields a faithful exact functor

$$
\mathbf{O}(X) \rightarrow \mathbf{S h}(X)
$$

such that

$$
\Gamma(X, \mathscr{M})=\Gamma\left(X, \mathscr{M}^{\mathbb{C}}\right) ;
$$

see (3.12) for the definition of the left hand side. Now, let $\mathscr{F}=\left(\mathscr{F}_{w}\right)$ be an object in $\mathbf{S h}(X)$. The vector spaces $\operatorname{End}\left(\mathscr{F}_{w}\right)$ form a projective system via the maps

$$
\operatorname{End}\left(\mathscr{F}_{x}\right) \rightarrow \operatorname{End}\left(\mathscr{F}_{w}\right), \quad f \mapsto i_{w, x}^{!}(f) .
$$

We set

$$
\operatorname{End}(\mathscr{F})={\underset{w}{w}}_{\lim } \operatorname{End}\left(\mathscr{F}_{w}\right)
$$

We say that an object $\mathscr{F}$ of $\mathbf{S h}(X)$ is a $\mathfrak{g}$-module if it is equipped with an algebra homomorphism

$$
\mathcal{U}(\mathfrak{g}) \rightarrow \operatorname{End}(\mathscr{F}) .
$$

For instance, for $\mathscr{M} \in \mathbf{M}^{T}\left(X^{\dagger}\right)$ the object $\left(\mathscr{M}^{\dagger}\right)^{\mathbb{C}}$ of $\mathbf{S h}(X)$ is a $\mathfrak{g}$-module via the algebra homomorphism

$$
\delta_{l}: \mathcal{U}(\mathfrak{g}) \rightarrow \Gamma\left(X^{\dagger}, \mathscr{D}_{X^{\dagger}}\right) .
$$

See the beginning of Section 4.3 .

Step 2. Next, we define $G$-modules in $\hat{\mathbf{O}}(X)$. A standard parabolic subgroup of $G$ is a group scheme of the form $P=Q \times{ }_{G_{0}} P_{0}$ with $P_{0}$ a parabolic subgroup of $G_{0}$. Here the morphism $Q \rightarrow G_{0}$ is the canonical one. We fix a subposet ${ }^{P} \mathfrak{S} \subset \mathfrak{S}$ such that for $w \in P_{\mathfrak{S}}$ the subscheme $\bar{X}_{w} \subset X$ is stable under the $P$-action and

$$
X=\underset{w \in P_{\mathfrak{S}}}{\underset{\lim _{w}}{ }} \bar{X}_{w}
$$

We say that an object $\mathscr{F}=\left(\mathscr{F}_{w}\right)$ of $\hat{\mathbf{O}}(X)$ has an algebraic $P$-action if $\mathscr{F}_{w}$ has the structure of a $P$-equivariant quasicoherent $\mathscr{O}_{\bar{X}_{w}}$-module for $w \in P_{\mathfrak{S}}$ and if the isomorphism $i_{w, x}^{*} \mathscr{F}_{x} \cong \mathscr{F}_{w}$ is $P$-equivariant for $w \leqslant x$. Finally, we say that $\mathscr{F}$ is a $G$-module if it is equipped with an action of the (abstract) group $G$ such that for any standard parabolic subgroup $P$, the $P$-action on $\mathscr{F}$ is algebraic.

We are interested in a family of $G$-modules $\mathscr{V}^{i}$ in $\hat{\mathbf{O}}(X)$ defined as follows. Fix a basis $\left(m_{i}\right)_{i \in \mathbb{N}}$ of $V(\nu)$ such that each $m_{i}$ is a weight vector of weight $\nu_{i}$ and $\nu_{j}>\nu_{i}$ implies $j<i$. By assumption we have $\nu_{0}=\nu$. For each $i$ let $V^{i}$ be the subspace of $V(\nu)$ spanned by the vectors $m_{j}$ for $j \leqslant i$. Then

$$
V^{0} \subset V^{1} \subset V^{2} \subset \cdots
$$


is a sequence of $B$-submodule of $V(\nu)$. We write $V^{\infty}=V(\nu)$. For $0 \leqslant i \leqslant \infty$ we define a $\mathscr{O}_{\mathfrak{X}}$-module $\mathscr{V}_{\mathfrak{X}}^{i}$ on $\mathfrak{X}$ such that for any open set $U \subset \mathfrak{X}$ we have

$$
\Gamma\left(U, \mathscr{V}_{\mathfrak{X}}^{i}\right)=\left\{f: p^{-1}(U) \rightarrow V^{i} \mid f\left(g b^{-1}\right)=b f(g), \quad g \in G_{\infty}, b \in B\right\},
$$

where $p: G_{\infty} \rightarrow \mathfrak{X}$ is the quotient map. Let $\mathscr{V}_{w}^{i}$ be the restriction of $\mathscr{V}_{\mathfrak{X}}^{i}$ to $\bar{X}_{w}$. Then $\left(\mathscr{V}_{w}^{i}\right)_{w \in \mathfrak{S}}$ is a flat $G$-module in $\hat{\mathbf{O}}(X)$. We will denote it by $\mathscr{V}^{i}$. Note that since $V(\nu)$ admits a $G$-action, the $G$-module $\mathscr{V}^{\infty} \in \hat{\mathbf{O}}(X)$ is isomorphic to the $G$-module $\mathscr{O}_{X} \otimes V(\nu)$ with $G$ acting diagonally. Therefore, for $\mathscr{M} \in \mathbf{M}_{0}^{\mu}(X)$ the projection formula yields a canonical isomorphism of vector spaces

$$
\begin{aligned}
\Gamma(\mathscr{M}) \otimes V(\nu) & =\Gamma\left(X, \mathscr{M}^{\dagger}\right) \otimes V(\nu) \\
& =\Gamma\left(X, \mathscr{M}^{\dagger} \otimes_{\mathscr{O}_{X}} \mathscr{V}^{\infty}\right) .
\end{aligned}
$$

On the other hand, we have

$$
\begin{aligned}
\Gamma\left(\Theta^{\nu}(\mathscr{M})\right) & =\Gamma\left(X,\left(\mathscr{M} \otimes_{\mathscr{O}_{X \dagger}} \pi^{*}\left(\mathscr{L}^{\nu}\right)\right)^{\dagger}\right) \\
& =\Gamma\left(X, \mathscr{M}^{\dagger} \otimes_{\mathscr{O}_{X}} \mathscr{L}^{\nu}\right) .
\end{aligned}
$$

Our goal is to compare the $\mathfrak{g}$-modules $\Gamma\left(\Theta^{\nu}(\mathscr{M})\right)$ and the direct factor $\theta^{\nu}(\Gamma(\mathscr{M}))$ of $\Gamma(\mathscr{M}) \otimes V(\nu)$. To this end, we first define in Step 3 a $\mathfrak{g}$-action on $\left(\mathscr{M}^{\dagger} \otimes_{\mathscr{O}_{X}} \mathscr{V}^{i}\right)^{\mathbb{C}}$ for each $i$, then we prove in Steps 4-6 that the inclusion

$$
\left(\mathscr{M}^{\dagger} \otimes_{\mathscr{O}_{X}} \mathscr{L}^{\nu}\right)^{\mathbb{C}} \rightarrow\left(\mathscr{M}^{\dagger} \otimes_{\mathscr{O}_{X}} \mathscr{V}^{\infty}\right)^{\mathbb{C}}
$$

induced by the inclusion $\mathscr{L}^{\nu}=\mathscr{V}^{0} \subset \mathscr{V}^{\infty}$ splits as a $\mathfrak{g}$-module homomorphism in $\operatorname{Sh}(X)$.

Step 3. Let $P$ be a standard parabolic subgroup of $G$, and let $\mathfrak{p}$ be its Lie algebra. Let $P_{\mathfrak{S}} \subset \mathfrak{S}$ be as in Step 2. The $P$-action on $\mathscr{V}^{i}$ yields a Lie algebra homomorphism

$$
\mathfrak{p} \rightarrow \operatorname{End}\left(\mathscr{V}_{w}^{i}\right), \quad \forall w \in P^{P} .
$$

Consider the $\mathfrak{g}$-action on $\left(\mathscr{M}^{\dagger}\right)^{\mathbb{C}}$ given by the map $\delta_{l}$ in A.12). Note that for $w \leqslant x$ in ${ }^{P_{\mathfrak{S}}}$, any element $\xi \in \mathfrak{p}$ maps a local section of $\mathscr{M}_{x}^{\dagger}$ supported on $\bar{X}_{w}$ to a local section of $\mathscr{M}_{x}^{\dagger}$ with the same property. In particular, for $w \in{ }^{P} \mathfrak{S}$ we have a Lie algebra homomorphism

$$
\mathfrak{p} \rightarrow \operatorname{End}\left(\left(\mathscr{M}_{w}^{\dagger} \otimes_{\mathscr{O}_{\bar{X}_{w}}} \mathscr{V}_{w}^{i}\right)^{\mathbb{C}}\right), \quad \xi \mapsto(m \otimes v \mapsto \xi m \otimes v+m \otimes \xi v),
$$

where $m$ denotes a local section of $\mathscr{M}_{w}^{\dagger}, v$ denotes a local section of $\mathscr{V}_{w}^{i}$. These maps are compatible with the restriction

$$
\operatorname{End}\left(\left(\mathscr{M}_{x}^{\dagger} \otimes_{\mathscr{O}_{\bar{X}_{x}}} \mathscr{V}_{x}^{i}\right)^{\mathbb{C}}\right) \rightarrow \operatorname{End}\left(\left(\mathscr{M}_{w}^{\dagger} \otimes_{\mathscr{O}_{\bar{X}_{w}}} \mathscr{V}_{w}^{i}\right)^{\mathbb{C}}\right), \quad f \mapsto i_{w, x}^{!}(f) .
$$

They yield a Lie algebra homomorphism

$$
\mathfrak{p} \rightarrow \operatorname{End}\left(\left(\mathscr{M}^{\dagger} \otimes_{\mathscr{O}_{X}} \mathscr{V}^{i}\right)^{\mathbb{C}}\right) .
$$

As $P$ varies, these maps glue together yielding a Lie algebra homomorphism

$$
\mathfrak{g} \rightarrow \operatorname{End}\left(\left(\mathscr{M}^{\dagger} \otimes_{\mathscr{O}_{X}} \mathscr{V}^{i}\right)^{\mathbb{C}}\right) .
$$

This defines a $\mathfrak{g}$-action on $\left(\mathscr{M}^{\dagger} \otimes_{\mathscr{O}_{X}} \mathscr{V}^{i}\right)^{\mathbb{C}}$ such that the obvious inclusions

$$
\left(\mathscr{M}^{\dagger} \otimes_{\mathscr{O}_{X}} \mathscr{V}^{0}\right)^{\mathbb{C}} \subset\left(\mathscr{M}^{\dagger} \otimes_{\mathscr{O}_{X}} \mathscr{V}^{1}\right)^{\mathbb{C}} \subset \ldots
$$

are $\mathfrak{g}$-equivariant. So A.15) is a $\mathfrak{g}$-module homomorphism. Note that the flatness of $\mathscr{V}^{i}$ yields an isomorphism in $\mathbf{O}(X)$ :

$$
\mathscr{M}^{\dagger} \otimes_{\mathscr{O}_{X}} \mathscr{V}^{i} / \mathscr{M}^{\dagger} \otimes_{\mathscr{O}_{X}} \mathscr{V}^{i-1} \cong \mathscr{M}^{\dagger} \otimes_{\mathscr{O}_{X}} \mathscr{L}^{\nu_{i}}
$$


Step 4. In order to show that the $\mathfrak{g}$-module homomorphism A.15 splits, we consider the generalized Casimir operator of $\mathfrak{g}$. Identify $\mathfrak{t}$ and $\mathfrak{t}^{*}$ via the pairing $\langle\bullet: \bullet\rangle$. Let $\rho^{\vee} \in \mathfrak{t}$ be the image of $\rho$. Let $h_{i}$ be a basis of $\mathfrak{t}_{0}$, and let $h^{i}$ be its dual basis in $\mathfrak{t}_{0}$ with respect to the pairing $\langle\bullet: \bullet\rangle$. For $\xi \in \mathfrak{g}_{0}$ and $n \in \mathbb{Z}$ we will abbreviate $\xi^{(n)}=\xi \otimes t^{n}$ and $\xi=\xi^{(0)}$. The generalized Casimir operator is given by the formal sum

$$
\mathfrak{C}=2 \rho^{\vee}+\sum_{i} h^{i} h_{i}+2 \partial \mathbf{1}+\sum_{i<j} e_{j i} e_{i j}+\sum_{n \geqslant 1} \sum_{i \neq j} e_{i j}^{(-n)} e_{j i}^{(n)}+\sum_{n \geqslant 1} \sum_{i} h^{i,(-n)} h_{i}^{(n)}
$$

see e.g., [Ka, Section 2.5]. Let $\delta_{l}(\mathfrak{C})$ be the formal sum given by applying $\delta_{l}$ term by term to the right hand side of (A.19). We claim that $\delta_{l}(\mathfrak{C})$ is a well defined element in $\Gamma\left(X^{\dagger}, \mathscr{D}_{X^{\dagger}}\right)$, i.e., the sum is finite at each point of $X^{\dagger}$. More precisely, let

$$
\Sigma=\left\{e_{i j} \mid i<j\right\} \cup\left\{e_{j i}^{(n)}, h_{i}^{(n)} \mid i \neq j, n \geqslant 1\right\},
$$

and let $e$ be the base point of $X^{\dagger}$. We need to prove that the sets

$$
\Sigma_{g}=\left\{\xi \in \Sigma \mid \delta_{l}(\xi)(g e) \neq 0\right\}, \quad g \in G,
$$

are finite. To show this, consider the adjoint action of $G$ on $\mathfrak{g}$

$$
\operatorname{Ad}: G \rightarrow \operatorname{End}(\mathfrak{g}), \quad g \mapsto \operatorname{Ad}_{g}
$$

and the $G$-action on $\mathscr{D}_{X^{\dagger}} \in \hat{\mathbf{O}}\left(X^{\dagger}\right)$ induced by the $G$-action on $X^{\dagger}$. The map $\delta_{l}$ is $G$-equivariant with respect to these actions. So for $\xi \in \mathfrak{g}$ and $g \in G$ we have

$$
\delta_{l}(\xi)(g e) \neq 0 \quad \Longleftrightarrow \quad \delta_{l}\left(\operatorname{Ad}_{g^{-1}}(\xi)\right)(e) \neq 0 .
$$

Furthermore, the right hand side holds if and only if $\operatorname{Ad}_{g^{-1}}(\xi) \notin \mathfrak{n}$. Therefore

$$
\Sigma_{g}=\left\{\xi \in \Sigma \mid \operatorname{Ad}_{g^{-1}}(\xi) \notin \mathfrak{n}\right\}
$$

is a finite set, the claim is proved. By consequence $\mathfrak{C}$ acts on the $\mathfrak{g}$-module $\left(\mathscr{M}^{\dagger}\right)^{\mathbb{C}}$ for any $\mathscr{M} \in \mathbf{M}^{T}\left(X^{\dagger}\right)$. Next, we claim that the action of $\mathfrak{C}$ on the $\mathfrak{g}$-module $\left(\mathscr{M}^{\dagger} \otimes_{\mathscr{O}_{X}} \mathscr{V}^{i}\right)^{\mathbb{C}}$ is also well defined. It is enough to prove this for $\left(\mathscr{M} \otimes_{\mathscr{O}_{X}} \mathscr{V}^{\infty}\right)^{\mathbb{C}}$. By (A.16) the action of $\mathfrak{C}$ on $\left(\mathscr{M} \otimes_{\mathscr{O}_{X}} \mathscr{V}^{\infty}\right)^{\mathbb{C}}$ is given by the operator

$$
\mathfrak{C} \otimes 1+1 \otimes \mathfrak{C}-\sum_{n \in \mathbb{Z}, i \neq j} e_{i j}^{(-n)} \otimes e_{j i}^{(n)}-\sum_{n \in \mathbb{Z}, i} h^{i,(-n)} \otimes h_{i}^{(n)} .
$$

Since for both $\mathscr{M}^{\dagger}$ and $\mathscr{V}^{\infty}$, at each point, there are only finitely many elements from $\Sigma$ which act nontrivially on it, the action of $\mathfrak{C}$ on the tensor product is well defined.

Step 5. Now, let us calculate the action of $\mathfrak{C}$ on $\left(\mathscr{M}^{\dagger} \otimes_{\mathscr{O}_{X}} \mathscr{L}^{\nu_{i}}\right)^{\mathbb{C}}$. We have

$$
\operatorname{Ad}_{g^{-1}}(\mathfrak{C})=\mathfrak{C}, \quad \forall g \in G .
$$

Therefore the global section $\delta_{l}(\mathfrak{C})$ is $G$-invariant and its value at $e$ is

$$
\delta_{l}(\mathfrak{C})(e)=\delta_{l}\left(2 \rho^{\vee}+\sum_{i} h^{i} h_{i}+2 \partial \mathbf{1}\right)(e) .
$$

On the other hand, the right $T$-action on $X^{\dagger}$ yields a map

$$
\delta_{r}: \mathfrak{t} \rightarrow \Gamma\left(X^{\dagger}, \mathscr{D}_{X^{\dagger}}\right) .
$$

Since the right $T$-action commutes with the left $G$-action, for any $h \in \mathfrak{t}$ the global section $\delta_{r}(h)$ is $G$-invariant. We have $\delta_{r}(h)(e)=-\delta_{l}(h)(e)$ because the left and right $T$-actions on the point $e$ are inverse to each other. Therefore the global 
sections $\delta_{l}(\mathfrak{C})$ and $\delta_{r}\left(-2 \rho^{\vee}+\sum_{i} h^{i} h_{i}+2 \partial \mathbf{1}\right)$ takes the same value at the point $e$. Since both of them are $G$-invariant, we deduce that

$$
\delta_{l}(\mathfrak{C})=\delta_{r}\left(-2 \rho^{\vee}+\sum_{i} h^{i} h_{i}+2 \partial \mathbf{1}\right) .
$$

Recall from Section 3.4 that for $\lambda \in \mathfrak{t}^{*}$ and $\mathscr{M} \in \mathbf{M}^{\lambda}(X)$ the operator $\delta_{r}\left(-2 \rho^{\vee}+\right.$ $\left.\sum_{i} h^{i} h_{i}+2 \partial \mathbf{1}\right)$ acts on $\mathscr{M}^{\dagger}$ by the scalar

$$
-\lambda\left(-2 \rho^{\vee}+\sum_{i} h^{i} h_{i}+2 \partial \mathbf{1}\right)=\|\lambda+\rho\|^{2}-\|\rho\|^{2} .
$$

Therefore $\mathfrak{C}$ acts on $\mathscr{M}^{\dagger}$ by the same scalar. In particular, for $\mathscr{M} \in \mathbf{M}^{\mu}(X)$ and $i \in \mathbb{N}$, the element $\mathfrak{C}$ acts on $\mathscr{M}^{\dagger} \otimes_{\mathscr{O}_{X}} \mathscr{L}^{\nu_{i}}$ by $\left\|\mu+\nu_{i}+\rho\right\|^{2}-\|\rho\|^{2}$. Note that the isomorphism A.18 is compatible with the $\mathfrak{g}$-actions. So $\mathfrak{C}$ also acts by $\left\|\mu+\nu_{i}+\rho\right\|^{2}-\|\rho\|^{2}$ on $\left(\mathscr{M}^{\dagger} \otimes_{\mathscr{O}_{X}} \mathscr{V}^{i} / \mathscr{M}^{\dagger} \otimes_{\mathscr{O}_{X}} \mathscr{V}^{i-1}\right) \mathbb{C}$.

Step 6 . Now, we can complete the proof of the proposition. First, we claim that

$$
\|\lambda+\rho\|^{2}-\|\rho\|^{2}=\left\|\mu+\nu_{i}+\rho\right\|^{2}-\|\rho\|^{2} \Longleftrightarrow \nu_{i}=\nu .
$$

The "if" part is trivial. For the "only if" part, we have by assumption

$$
\begin{aligned}
\|\mu+\nu+\rho\|^{2} & =\left\|\mu+\nu_{i}+\rho\right\|^{2} \\
& =\|\mu+\nu+\rho\|^{2}+\left\|\nu-\nu_{i}\right\|^{2}-2\left\langle\mu+\nu+\rho: \nu-\nu_{i}\right\rangle .
\end{aligned}
$$

Since $\nu-\nu_{i} \in \mathbb{N}^{+}$and $\mu+\nu+\rho=\lambda+\rho$ is antidominant, the term $-2\left\langle\lambda+\rho: \nu-\nu_{i}\right\rangle$ is positive. Hence the equality implies that $\left\|\nu-\nu_{i}\right\|^{2}=0$. So $\nu-\nu_{i}$ belongs to $\mathbb{N} \delta$. But $\langle\lambda+\rho: \delta\rangle=\kappa<0$. So we have $\nu=\nu_{i}$. This proves the claim in A.20). A direct consequence of this claim and of Step 5 is that the $\mathfrak{g}$-module monomorphism (A.15) splits. It induces an isomorphism of $\mathfrak{g}$-modules

$$
\Gamma\left(\mathscr{M}^{\dagger} \otimes_{\mathscr{O}_{X}} \mathscr{L}^{\nu}\right)=\operatorname{pr}_{\lambda} \Gamma\left(\mathscr{M}^{\dagger} \otimes_{\mathscr{O}_{X}} \mathscr{V}^{\infty}\right), \quad \mathscr{M} \in \mathbf{M}_{0}^{\mu}(X) .
$$

Finally, note that the vector spaces isomorphisms (A.13) and (A.14) are indeed isomorphisms of $\mathfrak{g}$-modules by the definition of the $\mathfrak{g}$-actions on $\left(\mathscr{M}^{\dagger} \otimes_{\mathscr{O}_{X}} \mathscr{V}^{\infty}\right)^{\mathbb{C}}$ and $\left(\mathscr{M}^{\dagger} \otimes_{\mathscr{O}_{X}} \mathscr{L}^{\nu}\right)^{\mathbb{C}}$. Therefore (A.21) yields an isomorphism of $\mathfrak{g}$-modules

$$
\Gamma\left(\Theta^{\nu}(\mathscr{M})\right)=\theta^{\nu}(\Gamma(\mathscr{M})) .
$$

Remark A.3. We have assumed $\mu+\rho$ regular in Proposition A.2 in order to have $\Gamma\left(\mathbf{M}_{0}^{\mu}(X)\right) \subset \tilde{\mathcal{O}}_{\kappa, \mu}$. It follows from Proposition A.2 that this inclusion still holds if $\mu+\rho$ is not regular. So Proposition A.2 makes sense without this regularity assumption, and the proof is the same in this case.

A.6. Proof of Proposition 4.2, By Proposition A.1 it is enough to prove the proposition in the case when $\lambda+\rho$ is not regular. Let $\omega_{i}, 0 \leqslant i \leqslant m-1$, be the fundamental weights in $\mathfrak{t}^{*}$. Let

$$
\nu=\sum \omega_{i}
$$

where the sum runs over all $i=0, \ldots, m-1$ such that $\left\langle\lambda+\rho: \alpha_{i}\right\rangle=0$. The weight $\nu$ is dominant. Let $\mu=\lambda-\nu$. Then $\mu+\rho$ is an antidominant weight. It is, moreover, regular, because we have

$$
\left\langle\mu+\rho: \alpha_{i}\right\rangle=\left\langle\lambda+\rho: \alpha_{i}\right\rangle-\left\langle\nu: \alpha_{i}\right\rangle<0, \quad 0 \leqslant i \leqslant m-1 .
$$

Let $\kappa^{\prime}=\langle\mu+\rho: \delta\rangle$. So Propositions A.1, A.2 and the equation A.9 imply that $\Gamma\left(\mathscr{A}_{w !}^{\lambda}\right)=\theta^{\nu}\left(N_{\kappa^{\prime}}(w \cdot \mu)\right), \quad \Gamma\left(\mathscr{A}_{w ! \bullet}^{\lambda}\right)=\theta^{\nu}\left(L_{\kappa^{\prime}}(w \cdot \mu)\right), \quad \Gamma\left(\mathscr{A}_{w \bullet}^{\lambda}\right)=\theta^{\nu}\left(\mathbf{D} N_{\kappa^{\prime}}(w \cdot \mu)\right)$. 
So parts (a) and (c) follow from the properties of the translation functor $\theta^{\nu}$ given in [Ku1, Proposition 1.7]. Part (b) follows from (a) and the equality (A.8).

\section{Appendix B. Proof of Proposition 5.4}

In this appendix, we prove Proposition 5.4. We will first study localization of deformed Verma modules; see Lemmas B.2 and B.3. Then we use them to deduce the parabolic version. In this appendix we will keep the notation of Section 5. In particular, recall that $j=j_{w}, f=f_{w}$, etc.

B.1. Deformed Verma modules. Fix $\lambda \in \Lambda$ and $w \in Q_{\mathfrak{S}}$. Let $n>0$ be an integer. For $v=x w \in \mathfrak{S}$ with $x \in \mathfrak{S}_{0}$, let $r_{x}: X_{v}^{\dagger} \rightarrow Y_{w}^{\dagger}$ be the canonical inclusion. We have $i_{v}=j \circ r_{x}$. The tensor product

$$
\mathscr{A}_{v}^{(n)}=\mathscr{A}_{v}^{\lambda} \otimes \mathscr{O}_{X_{v}^{\dagger}} r_{x}^{*} f^{*} \mathscr{I}^{(n)}
$$

is equal to $r_{x}^{*}\left(\mathscr{B}^{(n)}\right)$. By Lemma 5.3 it is an object of $\mathbf{M}_{h}^{\tilde{\lambda}}\left(X_{v}\right)$. We consider the following objects in $\mathbf{M}_{h}^{\tilde{\lambda}}(X)$ :

$$
\mathscr{A}_{v !}^{(n)}=i_{v !}\left(\mathscr{A}_{v}^{(n)}\right), \quad \mathscr{A}_{v ! \bullet}^{(n)}=i_{v ! \bullet}\left(\mathscr{A}_{v}^{(n)}\right), \quad \mathscr{A}_{v \bullet}^{(n)}=i_{v \bullet}\left(\mathscr{A}_{v}^{(n)}\right) .
$$

For $\mu \in{ }_{\kappa} \mathrm{t}^{*}$ we have defined the Verma module $N_{\kappa}(\mu)$ in Section 4.4. The deformed Verma module is the $\mathcal{U}_{\mathbf{k}}$-module given by

$$
N_{\mathbf{k}}(\mu)=\mathcal{U}(\mathfrak{g}) \otimes \mathcal{U}(\mathfrak{b}) R_{\mu+s \omega_{0}} .
$$

Here the $\mathfrak{b}$-module $R_{\mu+s \omega_{0}}$ is a rank one $R$-module over which $\mathfrak{t}$ acts by $\mu+s \omega_{0}$, and $\mathfrak{n}$ acts trivially. The deformed dual Verma module is

$$
\mathbf{D} N_{\mathbf{k}}(\mu)=\bigoplus_{\lambda \in \mathbf{k} \mathbf{t}^{*}} \operatorname{Hom}_{R}\left(N_{\mathbf{k}}(\mu)_{\lambda}, R\right)
$$

see (2.5). We will abbreviate

$$
N_{\mathbf{k}}^{(n)}(\mu)=N_{\mathbf{k}}(\mu)\left(\wp^{n}\right), \quad \mathbf{D} N_{\mathbf{k}}^{(n)}(\mu)=\mathbf{D} N_{\mathbf{k}}(\mu)\left(\wp^{n}\right) .
$$

For any $R^{(n)}$-module (resp. $R$-module) $M$ let $\mu\left(s^{i}\right): M \rightarrow M$ be the multiplication by $s^{i}$ and write $s^{i} M$ for the image of $\mu\left(s^{i}\right)$. We define a filtration

$$
F^{\bullet} M=\left(F^{0} M \supset F^{1} M \supset F^{2} M \supset \ldots\right)
$$

on $M$ by putting $F^{i} M=s^{i} M$. We say that it is of length $n$ if $F^{n} M=0$ and $F^{n-1} M \neq 0$. We set

$$
\operatorname{gr} M=\bigoplus_{i \geqslant 0} \operatorname{gr}^{i} M, \quad \operatorname{gr}^{i} M=F^{i} M / F^{i+1} M .
$$

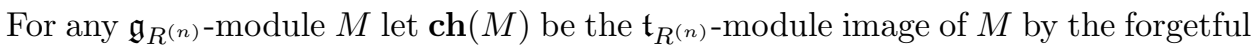
functor.

Lemma B.1. If $\lambda+\rho$ is antidominant, then we have an isomorphism of $\mathfrak{t}_{R^{(n)}}$ modules

$$
\operatorname{ch}\left(\Gamma\left(\mathscr{A}_{v \bullet}^{(n)}\right)\right)=\operatorname{ch}\left(\mathbf{D} N_{\mathbf{k}}^{(n)}(v \cdot \lambda)\right) .
$$

Proof. The proof is very similar to the proof of Proposition A.1. We will use the notation introduced there. 
Step 1. Consider the nowhere vanishing section $f^{s}$ of $\left(f^{*} \mathscr{I}^{(n)}\right)^{\dagger}$ over $Y_{w}$. Its restriction to $X_{v}$ yields an isomorphism

$$
\left(\mathscr{A}_{v}^{(n)}\right)^{\dagger} \cong \Omega_{X_{v}} \otimes_{\mathscr{O}_{X_{v}}} \mathscr{L}_{X_{v}}^{\lambda} \otimes R^{(n)} .
$$

Let $\omega$ be a nowhere vanishing section of $\Omega_{X_{v}}$, and let $t^{\lambda}$ be the nowhere vanishing section of $\mathscr{L}_{X_{v}}^{\lambda}$ over $X_{v}$ such that $t^{\lambda}(u \dot{v} b)=e^{-\lambda}(b)$ for $u \in N, b \in B$. Then the global section $\omega \otimes t^{\lambda} \otimes f^{s}$ of $\mathscr{A}_{v}^{(n)}$ defines an element

$$
\vartheta^{s} \in \hat{\Gamma}\left(\mathscr{A}_{v \bullet}^{(n)}\right)
$$

in the same way as $\vartheta$ is defined in the first step of the proof of Proposition A.1

Step 2. Let us show that

$$
\operatorname{ch}\left(\bar{\Gamma}\left(\mathscr{A}_{v \bullet}^{(n)}\right)\right)=\operatorname{ch}\left(\mathbf{D} N_{\mathbf{k}}^{(n)}(v \cdot \lambda)\right) .
$$

The proof is the same as in the second step of the proof of Proposition A.1. The right $\mathscr{D}_{X_{v}}^{\dagger}$-module structure on $\left(\mathscr{A}_{v}^{(n)}\right)^{\dagger}$ yields an isomorphism of sheaves of $\mathbb{C}$-vector spaces over $V_{l}^{y}$,

$$
\begin{aligned}
j_{l}^{y *}\left(i_{l *}^{v} \mathscr{O}_{X_{v}}\right) \otimes \mathcal{U}\left(\mathfrak{n}^{-}\left(\Pi^{-} \cap v\left(\Pi^{-}\right)\right) / \mathfrak{n}_{l}^{-}\right) \otimes R^{(n)} & \stackrel{\sim}{\rightarrow} j_{l}^{y *}\left(i_{l \bullet}^{v}\left(\mathscr{A}_{v}^{(n)}\right)^{\dagger}\right), \\
f \otimes p \otimes r & \mapsto\left(\left(\vartheta^{s} \cdot f\right) \cdot \delta_{l}(p)\right) r .
\end{aligned}
$$

This yields an isomorphism of $\mathfrak{t}_{R^{(n)}}$-modules

$$
\operatorname{ch}\left(\Gamma\left(\mathfrak{X}_{l}^{y}, i_{l \bullet}^{v}\left(\mathscr{A}_{v}^{(n)}\right)\right)\right)=\operatorname{ch}\left(\mathcal{U}\left(\mathfrak{n}^{-} / \mathfrak{n}_{l}^{-}\right) \otimes R_{v \cdot \lambda+s \omega_{0}}^{(n)}\right) .
$$

Therefore we have

$$
\operatorname{ch}\left(\hat{\Gamma}\left(\mathscr{A}_{v \bullet}^{(n)}\right)\right)=\operatorname{ch}\left(\left(亡_{l}^{\lim } \mathcal{U}\left(\mathfrak{n}^{-} / \mathfrak{n}_{l}^{-}\right)\right) \otimes R_{v \cdot \lambda+s \omega_{0}}^{(n)}\right)
$$

and

$$
\begin{aligned}
\operatorname{ch}\left(\bar{\Gamma}\left(\mathscr{A}_{v \bullet}^{(n)}\right)\right) & =\operatorname{ch}\left(\mathcal{U}\left(\mathfrak{n}^{-}\right) \otimes R_{v \cdot \lambda+s \omega_{0}}^{(n)}\right) \\
& =\operatorname{ch}\left(\mathbf{D} N_{\mathbf{k}}^{(n)}(v \cdot \lambda)\right)
\end{aligned}
$$

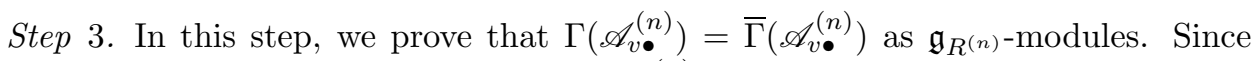
both of them are $\mathfrak{g}_{R^{(n)}}$-submodules of $\hat{\Gamma}\left(\mathscr{A}_{v \bullet}^{(n)}\right)$. It is enough to prove that they are equal as vector spaces. Consider the filtration $F^{\bullet}\left(\mathscr{A}_{v}^{(n)}\right)$ on $\mathscr{A}_{v}^{(n)}$. It is a filtration in $\mathbf{M}^{\tilde{\lambda}}\left(X_{v}\right)$ of length $n$ and

$$
\operatorname{gr}^{i}\left(\mathscr{A}_{v}^{(n)}\right)=\mathscr{A}_{v}^{\lambda}, \quad 0 \leqslant i \leqslant n-1 .
$$

Since $i_{v}$ is exact and

$$
R^{i} \Gamma\left(\mathscr{A}_{v \bullet}^{\lambda}\right)=R^{i} \bar{\Gamma}\left(\mathscr{A}_{v \bullet}^{\lambda}\right)=0, \quad \forall i>0,
$$

the functor $\Gamma \circ i_{v}$ commutes with the filtration. Therefore both the filtrations $F^{\bullet} \Gamma\left(\mathscr{A}_{v \bullet}^{(n)}\right)$ and $F^{\bullet} \bar{\Gamma}\left(\mathscr{A}_{v \bullet}^{(n)}\right)$ have length $n$ and

$$
\operatorname{gr}^{i} \Gamma\left(\mathscr{A}_{v \bullet}^{(n)}\right)=\Gamma\left(\mathscr{A}_{v \bullet}^{\lambda}\right), \quad \operatorname{gr}^{i} \bar{\Gamma}\left(\mathscr{A}_{v \bullet}^{(n)}\right)=\bar{\Gamma}\left(\mathscr{A}_{v \bullet}^{\lambda}\right), \quad 0 \leqslant i \leqslant n-1 .
$$


By Step 3 of the proof of Proposition A.1 we have $\Gamma\left(\mathscr{A}_{v}^{\lambda}\right)=\bar{\Gamma}\left(\mathscr{A}_{v}^{\lambda}\right)$. We deduce that all the sections in $\Gamma\left(\mathscr{A}_{v \bullet}^{(n)}\right)$ are $\mathfrak{t}$-finite and all the sections in $\bar{\Gamma}\left(\mathscr{A}_{v \bullet}^{(n)}\right)$ are supported on finite dimensional subschemes. This proves that

$$
\Gamma\left(\mathscr{A}_{v \bullet}^{(n)}\right)=\bar{\Gamma}\left(\mathscr{A}_{v \bullet}^{(n)}\right) .
$$

We are done by Step 2 .

Lemma B.2. If $\lambda+\rho$ is antidominant there is an isomorphism of $\mathfrak{g}_{R^{(n)}}$-modules

$$
\Gamma\left(\mathscr{A}_{v \bullet}^{(n)}\right)=\mathbf{D} N_{\mathbf{k}}^{(n)}(v \cdot \lambda) .
$$

Proof. Note that

$$
\begin{aligned}
\mathbf{D} N_{\mathbf{k}}^{(n)}(v \cdot \lambda) & =\bigoplus_{\mu \in \mathbf{k}^{\mathbf{t}^{*}}} \operatorname{Hom}_{R}\left(N_{\mathbf{k}}(v \cdot \lambda)_{\mu}, R\right)\left(\wp^{n}\right) \\
& =\bigoplus_{\mu \in \mathbf{k}^{\mathbf{t}^{*}}} \operatorname{Hom}_{R^{(n)}}\left(N_{\mathbf{k}}^{(n)}(v \cdot \lambda)_{\mu}, R^{(n)}\right) .
\end{aligned}
$$

For $\mu \in \mathbf{k}^{\mathfrak{t}^{*}}$ let $\Gamma\left(\mathscr{A}_{v}^{(n)}\right)_{\mu}$ be the weight space as defined in (2.2). By Lemma B.1 we have

$$
\Gamma\left(\mathscr{A}_{v \bullet}^{(n)}\right)=\bigoplus_{\mu \in \mathbf{k}^{\mathbf{t}^{*}}} \Gamma\left(\mathscr{A}_{v \bullet}^{(n)}\right)_{\mu}
$$

because the same equality holds for $\mathbf{D} N_{\mathbf{k}}^{(n)}(v \cdot \lambda)$. So we can consider the following $\mathfrak{g}_{R^{(n)}}$-module

$$
\mathrm{D} \Gamma\left(\mathscr{A}_{v \bullet}^{(n)}\right)=\bigoplus_{\mu \in \mathbf{k}^{\mathrm{t}^{*}}} \operatorname{Hom}_{R^{(n)}}\left(\Gamma\left(\mathscr{A}_{v \bullet}^{(n)}\right)_{\mu}, R^{(n)}\right) .
$$

It is enough to prove that we have an isomorphism of $\mathfrak{g}_{R^{(n)} \text {-modules }}$

$$
\mathbf{D} \Gamma\left(\mathscr{A}_{v \bullet}^{(n)}\right)=N_{\mathbf{k}}^{(n)}(v \cdot \lambda) .
$$

By (B.4) we have

$$
\operatorname{ch}\left(\mathrm{D} \Gamma\left(\mathscr{A}_{v \bullet}^{(n)}\right)\right)=\operatorname{ch}\left(\Gamma\left(\mathscr{A}_{v \bullet}^{(n)}\right)\right)
$$

Together with Lemma B.1, this yields an isomorphism of $R^{(n)}$-modules

$$
N_{\mathbf{k}}^{(n)}(v \cdot \lambda)_{v \cdot \lambda+s \omega_{0}}=\left(\mathbf{D} \Gamma\left(\mathscr{A}_{v \bullet}^{(n)}\right)\right)_{v \cdot \lambda+s \omega_{0}} .
$$

By the universal property of Verma modules, such an isomorphism induces a morphism of $\mathfrak{g}_{R^{(n)}}$-module

$$
\varphi: N_{\mathbf{k}}^{(n)}(v \cdot \lambda) \rightarrow \mathrm{D} \Gamma\left(\mathscr{A}_{v \bullet}^{(n)}\right) .
$$

We claim that for each $\mu \in \mathbf{k}^{\mathbf{t}^{*}}$ the $R^{(n)}$-module morphism

$$
\varphi_{\mu}: N_{\mathbf{k}}^{(n)}(v \cdot \lambda)_{\mu} \rightarrow\left(\mathbf{D} \Gamma\left(\mathscr{A}_{v \bullet}^{(n)}\right)\right)_{\mu}
$$

given by the restriction of $\varphi$ is invertible. Indeed, by Lemma B.1 and (B.5), we have

So

$$
\operatorname{ch}\left(\mathbf{D} \Gamma\left(\mathscr{A}_{v \bullet}^{(n)}\right)\right)=\operatorname{ch}\left(\mathbf{D} N_{\mathbf{k}}^{(n)}(v \cdot \lambda)\right)=\operatorname{ch}\left(N_{\mathbf{k}}^{(n)}(v \cdot \lambda)\right) .
$$

$$
N_{\mathbf{k}}^{(n)}(v \cdot \lambda)_{\mu}=\left(\mathbf{D} \Gamma\left(\mathscr{A}_{v \bullet}^{(n)}\right)\right)_{\mu}
$$

as $R^{(n)}$-modules. On the other hand, Proposition 4.2 yields that the map

$$
\varphi(\wp)=\varphi \otimes_{R^{(n)}}\left(R^{(n)} / \wp R^{(n)}\right): N_{\kappa}(v \cdot \lambda) \rightarrow \mathbf{D} \Gamma\left(\mathscr{A}_{v \bullet}^{\lambda}\right)
$$


is an isomorphism of $\mathfrak{g}$-modules. So $\varphi_{\mu}(\wp)$ is also an isomorphism. Since the $R^{(n)}$-modules $N_{\mathbf{k}}^{(n)}(v \cdot \lambda)_{\mu}$ and $\left(\mathbf{D} \Gamma\left(\mathscr{A}_{v \bullet}^{(n)}\right)\right)_{\mu}$ are finitely generated, Nakayama's lemma implies that $\varphi_{\mu}$ is an isomorphism. So $\varphi$ is an isomorphism. The lemma is proved.

Lemma B.3. If $\lambda+\rho$ is antidominant and $v$ is a shortest element in $v \mathfrak{S}(\lambda)$, then there is an isomorphism of $\mathfrak{g}_{R^{(n)}}$-modules

$$
\Gamma\left(\mathscr{A}_{v !}^{(n)}\right)=N_{\mathbf{k}}^{(n)}(v \cdot \lambda) .
$$

Proof. We abbreviate $\nu=v \cdot \lambda$. The lemma will be proved in three steps.

Step 1. Recall the character map from (4.4). Note that since $\Gamma$ and $i_{!}$are exact, and

$$
\operatorname{gr} \mathscr{A}_{v}^{(n)}=\left(\mathscr{A}_{v}^{\lambda}\right)^{\oplus n},
$$

we have an isomorphism of $\mathfrak{t}$-modules

$$
\operatorname{gr} \Gamma\left(\mathscr{A}_{v !}^{(n)}\right)=\Gamma\left(\mathscr{A}_{v !}^{\lambda}\right)^{\oplus n} .
$$

Next, since the action of $s$ on $\Gamma\left(\mathscr{A}_{v !}^{(n)}\right)$ is nilpotent, for any $\mu \in \mathfrak{t}^{*}$ we have

$$
\operatorname{dim}_{\mathbb{C}}\left(\Gamma\left(\mathscr{A}_{v !}^{(n)}\right)_{\tilde{\mu}}\right)=\operatorname{dim}_{\mathbb{C}}\left(\left(\operatorname{gr} \Gamma\left(\mathscr{A}_{v !}^{(n)}\right)\right)_{\tilde{\mu}}\right) .
$$

We deduce that as a $\mathfrak{t}$-module $\Gamma\left(\mathscr{A}_{v !}^{(n)}\right)$ is a generalized weight module and

$$
\operatorname{ch}\left(\Gamma\left(\mathscr{A}_{v !}^{(n)}\right)\right)=\operatorname{ch}\left(\operatorname{gr} \Gamma\left(\mathscr{A}_{v !}^{(n)}\right)\right)=n \operatorname{ch} \Gamma\left(\mathscr{A}_{v !}^{\lambda}\right) .
$$

On the other hand, we have the following isomorphism of $\mathfrak{t}$-modules

$$
\operatorname{gr} N_{\mathbf{k}}^{(n)}(\nu)=N_{\kappa}(\nu)^{\oplus n} \text {. }
$$

Therefore we have

$$
\operatorname{ch}\left(N_{\mathbf{k}}^{(n)}(\nu)\right)=n \operatorname{ch}\left(N_{\kappa}(\nu)\right) .
$$

Since $\Gamma\left(\mathscr{A}_{v !}^{\lambda}\right)=N_{\kappa}(\nu)$ as $\mathfrak{g}$-modules by Proposition 4.2, this yields

$$
\operatorname{ch}\left(\Gamma\left(\mathscr{A}_{v !}^{(n)}\right)\right)=\operatorname{ch}\left(N_{\mathbf{k}}^{(n)}(\nu)\right) .
$$

Further, we claim that there is an isomorphism of $R^{(n)}$-module

$$
\Gamma\left(\mathscr{A}_{v !}^{(n)}\right)_{\tilde{\mu}}=N_{\mathbf{k}}^{(n)}(\nu)_{\tilde{\mu}}, \quad \forall \mu \in \mathfrak{t}^{*} .
$$

Note that $\Gamma\left(\mathscr{A}_{v !}^{(n)}\right)_{\tilde{\mu}}$ is indeed an $R^{(n)}$-module because the action of $s$ on $\Gamma\left(\mathscr{A}_{v !}^{(n)}\right)$ is nilpotent. To prove the claim, it suffices to notice that for any finitely generated $R^{(n)}$-modules $M, M^{\prime}$ we have that $M$ is isomorphic to $M^{\prime}$ as $R^{(n)}$-modules if and only if $\operatorname{gr}^{i} M=\operatorname{gr}^{i} M^{\prime}$ for all $i$. So the claim follows from the isomorphisms of t-modules (B.6), (B.8) and Proposition 4.2 .

Step 2. In this step, we prove that as a $\mathfrak{t}_{R^{(n)} \text {-module }}$

$$
\Gamma\left(\mathscr{A}_{v !}^{(n)}\right)_{\tilde{\nu}}=R_{\nu+s \omega_{0}}^{(n)}
$$

where $R_{\nu+s \omega_{0}}^{(n)}$ is the rank one $R^{(n)}$-module over which $\mathfrak{t}$ acts by the weight $\nu+s \omega_{0}$. Let us consider the canonical morphisms in $\mathbf{M}_{h}^{\tilde{\lambda}}(X)$ :

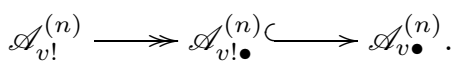


Since $\Gamma$ is exact on $\mathbf{M}^{\tilde{\lambda}}(X)$, we deduce the following chain of $\mathfrak{g}_{R^{(n)}}$-module morphisms

$$
\Gamma\left(\mathscr{A}_{v !}^{(n)}\right) \stackrel{\alpha}{\longrightarrow} \Gamma\left(\mathscr{A}_{v ! \bullet}^{(n)}\right) \stackrel{\beta}{\longrightarrow} \Gamma\left(\mathscr{A}_{v \bullet}^{(n)}\right) .
$$

Consider the following $\mathfrak{t}_{R^{(n)}}$-morphisms given by the restrictions of $\alpha, \beta$

$$
\Gamma\left(\mathscr{A}_{v !}^{(n)}\right)_{\tilde{\nu}} \stackrel{\alpha_{\nu}}{\longrightarrow} \Gamma\left(\mathscr{A}_{v ! \bullet}^{(n)}\right)_{\tilde{\nu}} \stackrel{\beta_{\nu}}{\longrightarrow} \Gamma\left(\mathscr{A}_{v \bullet}^{(n)}\right)_{\tilde{\nu}} .
$$

We claim that $\alpha_{\nu}$ and $\beta_{\nu}$ are isomorphisms. Note that by (B.7) we have

$$
\operatorname{dim}_{\mathbb{C}} \Gamma\left(\mathscr{A}_{v !}^{(n)}\right)_{\tilde{\nu}}=n \operatorname{dim}_{\mathbb{C}} \Gamma\left(\mathscr{A}_{v !}^{\lambda}\right)_{\tilde{\nu}}=n .
$$

By Lemma B.2 we also have $\operatorname{dim}_{\mathbb{C}} \Gamma\left(\mathscr{A}_{v \bullet}^{(n)}\right)_{\tilde{\nu}}=n$. Next, consider the exact sequence in $\mathbf{M}_{h}^{\tilde{\lambda}}\left(X_{v}\right)$,

$$
0 \rightarrow F^{i+1} \mathscr{A}_{v}^{(n)} \rightarrow F^{i} \mathscr{A}_{v}^{(n)} \rightarrow \operatorname{gr}^{i} \mathscr{A}_{v}^{(n)} \rightarrow 0 .
$$

Applying the functor $i_{v ! \bullet}$ to it yields a surjective morphism

$$
i_{v ! \bullet}\left(F^{i} \mathscr{A}_{v}^{(n)}\right) / i_{v ! \bullet}\left(F^{i+1} \mathscr{A}_{v}^{(n)}\right) \rightarrow i_{v ! \bullet}\left(\operatorname{gr}^{i} \mathscr{A}_{v}^{(n)}\right) .
$$

Since $i_{v ! \bullet}\left(F^{i} \mathscr{A}_{v}^{(n)}\right)=F^{i}\left(i_{v ! \bullet}\left(\mathscr{A}_{v}^{(n)}\right)\right)$ and $\operatorname{gr}^{i} \mathscr{A}_{v}^{(n)}=\mathscr{A}_{v}^{\lambda}$, we deduce a surjective morphism

$$
\operatorname{gr}^{i} \mathscr{A}_{v ! \bullet}^{(n)} \rightarrow \mathscr{A}_{v ! \bullet}^{\lambda}, \quad 0 \leqslant i \leqslant n-1 .
$$

Applying the exact functor $\Gamma$ to this morphism and summing over $i$ gives a surjective morphism of $\mathfrak{g}$-modules

$$
\gamma: \operatorname{gr} \Gamma\left(\mathscr{A}_{v ! \bullet}^{(n)}\right) \rightarrow \Gamma\left(\mathscr{A}_{v ! \bullet}^{\lambda}\right)^{\oplus n} .
$$

Since $v$ is minimal in $v \mathfrak{S}(\lambda)$, by Proposition 4.2 (c) the right hand side is equal to $L_{\kappa}(\nu)$. We deduce from the surjectivity of $\gamma$ that

$$
\begin{aligned}
\operatorname{dim}_{\mathbb{C}} \Gamma\left(\mathscr{A}_{v ! \bullet}^{(n)}\right)_{\tilde{\nu}} & =\operatorname{dim}_{\mathbb{C}} \operatorname{gr} \Gamma\left(\mathscr{A}_{v ! \bullet}^{(n)}\right)_{\tilde{\nu}} \\
& \geqslant \operatorname{dim}_{\mathbb{C}}\left(L_{\kappa}(\nu)_{\tilde{\nu}}\right)^{\oplus n} \\
& =n .
\end{aligned}
$$

It follows that the epimorphism $\alpha_{\nu}$ and the monomorphism $\beta_{\nu}$ are isomorphisms.

The claim is proved. So we have an isomorphisms of $\mathfrak{t}_{R^{(n)}}$-modules

$$
\beta_{\nu} \circ \alpha_{\nu}: \Gamma\left(\mathscr{A}_{v !}^{(n)}\right)_{\tilde{\nu}} \rightarrow \Gamma\left(\mathscr{A}_{v \bullet}^{(n)}\right)_{\tilde{\nu}}
$$

In particular, we deduce an isomorphisms of $\mathfrak{t}_{R^{(n)}}$-modules

$$
\Gamma\left(\mathscr{A}_{v !}^{(n)}\right)_{\nu+s \omega_{0}} \rightarrow \Gamma\left(\mathscr{A}_{v \bullet}^{(n)}\right)_{\nu+s \omega_{0}}
$$

because

$$
\Gamma\left(\mathscr{A}_{v \sharp}^{(n)}\right)_{\nu+s \omega_{0}} \subset \Gamma\left(\mathscr{A}_{v \sharp}^{(n)}\right)_{\tilde{\nu}}, \quad \text { for } \sharp=!, \bullet .
$$

By Lemma B.2, we have

$$
\Gamma\left(\mathscr{A}_{v \bullet}^{(n)}\right)_{\nu+s \omega_{0}}=R_{\nu+s \omega_{0}}^{(n)} .
$$

We deduce an isomorphism of $\mathfrak{t}_{R^{(n)} \text {-modules }}$

$$
\Gamma\left(\mathscr{A}_{v !}^{(n)}\right)_{\nu+s \omega_{0}}=R_{\nu+s \omega_{0}}^{(n)} .
$$


Step 3. By the universal property of Verma modules and Step 2, there exists a morphism of $\mathfrak{g}_{R^{(n)}}$-modules

$$
\varphi: N_{\mathbf{k}}^{(n)}(\nu) \rightarrow \Gamma\left(\mathscr{A}_{v !}^{(n)}\right) .
$$

For any $\mu \in \mathfrak{t}^{*}$ this map restricts to a morphism of $R^{(n)}$-modules

$$
\varphi_{\mu}: N_{\mathbf{k}}^{(n)}(\nu)_{\tilde{\mu}} \rightarrow \Gamma\left(\mathscr{A}_{v !}^{(n)}\right)_{\tilde{\mu}} .
$$

By Step 1 , the $R^{(n)}$-modules on the two sides are finitely generated and they are isomorphic. Further, the induced morphism

$$
\varphi(\wp): N_{\kappa}(\nu) \rightarrow \Gamma\left(\mathscr{A}_{v !}^{\lambda}\right)
$$

is an isomorphism by Proposition 4.2. So by Nakayama's lemma, the morphism $\varphi_{\mu}$ is an isomorphism for any $\mu$. Therefore $\varphi$ is an isomorphism. The lemma is proved.

Remark B.4. The hypothesis that $v$ is a shortest element in $v \mathfrak{S}(\lambda)$ is probably not necessary but this is enough for our purpose.

B.2. Proof of Proposition 5.4. Consider the canonical embedding $r: X_{w}^{\dagger} \rightarrow Y_{w}^{\dagger}$. We claim that the adjunction map yields a surjective morphism

$$
r ! r^{*}\left(\mathscr{B}^{(n)}\right) \rightarrow \mathscr{B}^{(n)} .
$$

Indeed, an easy induction shows that it is enough to prove that $\operatorname{gr}^{i}\left(r_{!} r^{*}\left(\mathscr{B}^{(n)}\right)\right) \rightarrow$ $\operatorname{gr}^{i} \mathscr{B}^{(n)}$ is surjective for each $i$. Since the functors $r_{!}, r_{*}$ are exact and $\operatorname{gr}^{i} \mathscr{B}^{(n)}=\mathscr{B}$, this follows from Lemma 4.4 (a). Note that $r^{*}\left(\mathscr{B}^{(n)}\right)=\mathscr{A}_{w}^{(n)}$. So the image of (B.11) by the exact functor $\Gamma \circ j$ ! is a surjective morphism

$$
\Gamma\left(\mathscr{A}_{w !}^{(n)}\right) \rightarrow \Gamma\left(\mathscr{B}_{!}^{(n)}\right) .
$$

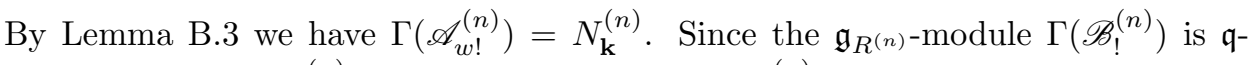
locally finite and $M_{\mathbf{k}}^{(n)}$ is the largest quotient of $N_{\mathbf{k}}^{(n)}$ in $\mathcal{O}_{\mathbf{k}}$, the morphism (B.12) induces a surjective morphism

$$
\varphi: M_{\mathbf{k}}^{(n)} \rightarrow \Gamma\left(\mathscr{B}_{!}^{(n)}\right) .
$$

Further, by Proposition 4.6(b) the map

$$
\varphi(\wp): M_{\kappa} \rightarrow \Gamma\left(\mathscr{B}_{!}\right)
$$

is an isomorphism. The same argument as in Step 1 of the proof of Lemma B.3 shows that for each $\mu \in \mathfrak{t}^{*}$ the generalized weight spaces $\left(M_{\mathbf{k}}^{(n)}\right)_{\tilde{\mu}}$ and $\Gamma\left(\mathscr{B}_{!}^{(n)}\right)_{\tilde{\mu}}$ are isomorphic as $R^{(n)}$-modules. We deduce that $\varphi$ is an isomorphism by Nakayama's lemma. This proves the first equality. The proof for the second equality is similar. We consider the adjunction map

$$
\mathscr{B}^{(n)} \rightarrow r_{\bullet} r^{*}\left(\mathscr{B}^{(n)}\right) .
$$

It is injective by Lemma 4.4(b) and the same arguments as above. So by applying the exact functor $\Gamma \circ j_{\bullet}$, we get an injective morphism

$$
\varphi^{\prime}: \Gamma\left(\mathscr{B}_{\bullet}^{(n)}\right) \rightarrow \mathbf{D} M_{\mathbf{k}}^{(n)} .
$$

Again, by using Proposition 4.6(b) and Nakayama's lemma, we prove that $\varphi^{\prime}$ is an isomorphism. 


\section{INDEX OF NOTATION}

1.1: $\mathcal{C} \cap R$-proj, $R^{\prime} \mathcal{C}$.

1.2: $\mathcal{C}^{\Delta}, \Delta, \nabla, D^{\vee}$.

1.3: $R=\mathbb{C}[[s]], \wp, K, M\left(\wp^{i}\right), M_{K}, f\left(\wp^{i}\right), f_{K}, \mathcal{C}(\wp), \mathcal{C}_{K}$.

1.4: $J^{i} D(\wp)$.

1.5: $F(\wp)$.

2.1: $G_{0}, B_{0}, T_{0}, \mathfrak{g}_{0}, \mathfrak{b}_{0}, \mathfrak{t}_{0}, \mathfrak{g}, \mathfrak{t}, \mathbf{1}, \partial, c, \kappa=c+m, \mathfrak{a}_{R}, \mathcal{U}_{\kappa}, M_{\lambda}, \mathfrak{t}_{0}^{*}, \mathfrak{t}^{*}, \delta, \omega_{0}$, $\epsilon_{i},\langle\bullet: \bullet\rangle,\|h\|^{2}, \kappa^{\mathfrak{t}^{*}}, a, z, \Pi, \Pi_{0}, \Pi^{+}, \Pi_{0}^{+}, \alpha_{i}, \mathfrak{S}, \mathfrak{S}_{0}, w \cdot \lambda, \rho_{0}, \rho, \mathfrak{S}(\lambda)$, $l: \mathfrak{S} \rightarrow \mathbb{N}$.

2.2: $\mathfrak{q}, \mathfrak{l}, \Lambda^{+}, M_{\kappa}(\lambda), L_{\kappa}(\lambda), \mathbf{c}, \mathbf{k}, \mathcal{U}_{\mathbf{k}}, M_{\mathbf{k}}(\lambda), \lambda_{s}, \mathbf{k}^{\mathfrak{t}^{*}}$.

2.3: $\sigma, \mathbf{D} M_{\mathbf{k}}(\lambda), J^{i} M_{\kappa}(\lambda)$.

2.4: $\mathcal{O}_{\mathbf{k}}, \mathcal{O}_{\kappa},{ }_{\kappa}^{r} t^{*},{ }_{\mathbf{k}}^{\mathrm{t}^{*}},{ }^{r} \mathcal{O}_{\kappa},{ }^{r} \mathcal{O}_{\mathbf{k}},{ }^{r} \Lambda^{+},{ }^{r} P_{\kappa}(\lambda), L_{\mathbf{k}}(\lambda),{ }^{r} P_{\mathbf{k}}(\lambda)$.

2.5: $\mathcal{P}_{n}, \preceq, \unlhd, E, \mathcal{E}_{\kappa}, \mathfrak{g}^{\prime}, \mathcal{E}_{\mathbf{k}}, P_{\mathbf{k}}(E), P_{\kappa}(E)$.

2.6: $\mathcal{A}_{\mathbf{k}}, \mathcal{A}_{\kappa}, \Delta_{\mathbf{k}}, \Delta_{\kappa}$.

2.7: D.

2.9: $\mathscr{H}_{\mathbf{v}}, \mathbf{S}_{\mathbf{v}}, \mathcal{A}_{\mathbf{v}}, W_{\mathbf{v}}(\lambda), \Delta_{\mathbf{v}}$.

2.10: $v=\exp (2 \pi i / \kappa), \mathbf{v}=\exp (2 \pi i / \mathbf{k}), J^{i} W_{v}(\lambda)$.

2.11: $\mathbf{H}_{1 / \kappa}, \mathcal{B}_{\kappa}, B_{\kappa}(\lambda), \mathfrak{E}_{\kappa}, \mathbf{H}_{1 / \mathbf{k}}, \mathcal{B}_{\mathbf{k}}, \mathfrak{E}_{\mathbf{k}}, B_{\mathbf{k}}(\lambda)$.

3.1: $\mathscr{O}_{Z}, \mathbf{O}(Z), f_{*}, f^{*}, f^{!}, \mathscr{D}_{Z}, \mathbf{M}(Z), \Omega_{Z}, \mathscr{D}_{Y \rightarrow Z}, \mathscr{M}^{\mathscr{O}}, \mathbf{M}\left(Z, Z^{\prime}\right), i^{*}, i_{\bullet}, i^{!}$.

3.2: $\mathbf{M}_{h}(Z), \mathbb{D}, i_{!}, i_{!}$.

3.3: $\mathbf{M}^{T}(Z), \mathbf{M}^{T}(X, Z)$.

3.4: $\mathscr{M}^{\dagger}, \mathscr{D}_{Z}^{\dagger}, \delta_{r}, \mathfrak{m}_{\lambda}, \mathbf{M}^{\lambda}(Z), \mathbf{M}^{\tilde{\lambda}}(Z), \mathbf{M}^{\lambda}(X, Z), \mathbf{M}^{\tilde{\lambda}}(X, Z), \mathbf{M}\left(\mathscr{D}_{Z}^{\dagger}\right)$.

3.5: $\mathbf{M}_{h}^{T}\left(Z^{\dagger}\right), \mathbf{M}_{h}^{\lambda}(Z), \mathbf{M}_{h}^{\tilde{\lambda}}(Z), \mathbb{D}^{\prime}, \mathscr{L}_{Z}^{\lambda}, \Theta^{\lambda}, \mathbb{D}^{\lambda}=\mathbb{D}^{\lambda}, \mathscr{D}_{Z}^{\lambda}$.

3.6: $2 \lim \mathcal{C}_{\alpha}, 2 \lim _{\longleftarrow} \mathcal{C}_{\alpha}$.

3.7: $X=\lim _{\longrightarrow} X_{\alpha}, \mathbf{O}(X), \Gamma(X, \mathscr{M})($ for $\mathscr{M} \in \mathbf{O}(X))$, $\hat{\mathbf{O}}(X), \mathscr{O}_{X},-\otimes_{\mathscr{O}_{X}} \mathscr{F}$, $\Gamma(X, \vec{F})($ for $\mathscr{F} \in \hat{\mathbf{O}}(X))$.

3.8: $\mathbf{M}(X)$ (with $X$ an ind-scheme), $\mathscr{M}^{\mathscr{O}}, \Gamma(X, \mathscr{M})$ (for $\left.\mathscr{M} \in \mathbf{M}(X)\right), \mathbf{M}^{T}(X)$, $\mathbf{M}^{\lambda}(X), \mathbf{M}^{\tilde{\lambda}}(X), \Gamma(\mathscr{M})\left(\right.$ for $\left.\mathscr{M} \in \mathbf{M}^{\tilde{\lambda}}(X)\right), \mathbf{M}_{h}^{\lambda}(X), \mathbf{M}_{h}^{\tilde{\lambda}}(X), i_{!}, i_{\bullet}$.

3.9: $\mathscr{D}_{X}$ (with $X$ a formally smooth ind-scheme).

4.1: $G, B, Q, T, N, \mathfrak{g}, \mathfrak{b}, \mathfrak{n}$.

4.2: $X=G / B, X^{\dagger}=G / N, \pi: X^{\dagger} \rightarrow X, X_{w}, \dot{w}, \bar{X}_{w}$.

4.3: $\delta_{l}: \mathcal{U}(\mathfrak{g}) \rightarrow \Gamma\left(X^{\dagger}, \mathscr{D}_{X^{\dagger}}\right), \mathbf{M}(\mathfrak{g}), \Gamma$

4.4: $M_{\tilde{\lambda}}, \operatorname{ch}(M), \tilde{\mathcal{O}}, \tilde{\mathcal{O}}_{\kappa}, N_{\kappa}(\lambda), L_{\kappa}(\lambda), \Lambda, \mathscr{A}_{w}^{\lambda}, i_{w}, \mathscr{A}_{w !}^{\lambda}, \mathscr{A}_{w ! \bullet}^{\lambda}, \mathscr{A}_{w \bullet}^{\lambda}$.

4.5: ${ }^{Q} \mathfrak{S}, w_{0}, Y_{w}, \bar{Y}_{w}, j_{w}, \mathscr{B}_{w}^{\lambda}, \mathscr{B}_{w !}^{\lambda}, \mathscr{B}_{w ! \bullet}^{\lambda}, \mathscr{B}_{w \bullet}^{\lambda}, r: X_{w}^{\dagger} \rightarrow Y_{w}^{\dagger}$.

5.1: $\mathcal{C}_{R}, \mu_{M}$, for,$F_{R}$.

5.2: $Q^{\prime}, f_{w}$.

5.3: $j, f, \mathscr{B}, \mathscr{B}_{!}, \mathscr{I}^{(n)}, x^{s}, f^{s}, \mathscr{B}^{(n)}, \mathscr{B}_{!}^{(n)}, \mathscr{B}_{!}^{(n)}, \mathscr{B}_{\bullet}^{(n)}$.

5.4: $M_{\kappa}, M_{\mathbf{k}}, M_{\mathbf{k}}^{(n)}, \mathbf{D} M_{\mathbf{k}}^{(n)}$.

5.5: $\psi(a, n), \pi^{a}(\mathscr{B}), J^{i}\left(\mathscr{B}_{!}\right)$. 
6.1: $\operatorname{MHM}(Z), W^{\bullet} \mathscr{M},(k), \operatorname{Perv}(Z), \varrho$.

6.2: $\mathbf{M}_{0}^{\lambda}\left(\bar{X}_{v}\right), \mathrm{DR}, \mathbf{M H M}_{0}\left(\bar{X}_{v}\right), \eta, \tilde{\mathscr{A}}_{w}^{\lambda}, \tilde{\mathscr{A}}_{w !}^{\lambda}, \tilde{\mathscr{A}}_{w ! \bullet}^{\lambda}, \tilde{\mathscr{B}}_{w}^{\lambda}, \tilde{\mathscr{B}}_{w !}^{\lambda}, \tilde{\mathscr{B}}_{w ! \bullet}^{\lambda}, q, \mathscr{H}_{q}(\mathfrak{S})$, $T_{w}, \Psi, P_{x, w}$,

A.1: $\Pi^{-}, \mathfrak{n}(\Upsilon), \mathfrak{n}^{-}(\Upsilon), \Pi_{l}^{-}, \mathfrak{n}_{l}^{-}, N^{-}, N_{l}^{-}, \mathfrak{X}, \mathfrak{X}^{\dagger}, \mathfrak{X}^{w}, \mathfrak{X}_{l}^{w}, \mathfrak{X}_{l}^{w \dagger}, p_{l_{1} l_{2}}, p_{l}$.

A.2: $\mathbf{H}^{\tilde{\lambda}}\left(\mathfrak{X}^{y}, \bar{X}_{w}\right), \mathbf{H}^{\tilde{\lambda}}\left(\bar{X}_{w}\right), \mathbf{H}^{\tilde{\lambda}}(X)$.

A.3: $\hat{\Gamma}(\mathscr{M}), \bar{\Gamma}(\mathscr{M})$.

A.5: $\tilde{\mathcal{O}}_{\kappa, \lambda}, \operatorname{pr}_{\lambda}, V(\nu), \theta^{\nu}, \mathscr{L}^{\lambda}, \Theta^{\lambda-\mu}$.

B.1: $r_{x}, \mathscr{A}_{v}^{(n)}, \mathscr{A}_{v !}^{(n)}, \mathscr{A}_{v ! \bullet}^{(n)}, \mathscr{A}_{v \bullet}^{(n)}, N_{\mathbf{k}}(\mu), R_{\mu+s \omega_{0}}, \mathbf{D} N_{\mathbf{k}}(\mu), N_{\mathbf{k}}^{(n)}(\mu), \mathbf{D} N_{\mathbf{k}}^{(n)}(\mu)$, $s^{i} M, F^{\bullet} M, \operatorname{gr} M, \operatorname{ch}(M)$.

\section{ACKNOWLEDGEMENT}

The author deeply thanks her advisor Eric Vasserot for many helpful discussions, lots of useful remarks and for his substantial support.

\section{REFERENCES}

[An] H. H. Andersen, The strong linkage principle for quantum groups at roots of 1, J. Algebra 260 (2003), no. 1, 2-15. MR1973573 (2004c:17024)

[Ar] S. Ariki, Graded q-Schur algebras, arxiv: 0903.3453

[B] A. Beilinson, How to glue perverse sheaves, $K$-theory, arithmetic and geometry (Moscow, 1984-1986), 42-51, Lecture Notes in Math., 1289, Springer, Berlin, 1987. MR923134 (89b:14028)

[BB] A. Beilinson, J. Bernstein, A proof of Jantzen conjectures, I. M. Gel'fand Seminar, 1-50, Adv. Soviet Math., 16, Part 1, Amer. Math. Soc., Providence, RI, 1993. MR.1237825 (95a:22022)

[BBD] A. Beilinson, J. Bernstein, P. Deligne, Faisceaux pervers, Analysis and topology on singular spaces, I (Luminy, 1981), 5-171, Astérisque, 100, Soc. Math. France, Paris, 1982. MR.751966 (86g:32015)

[BD] A. Beilinson, V. Drinfeld, Quantization of Hitchin's integrable system and Hecke eigensheaves, available at http://www.math.harvard.edu/〜 gaitsgde/grad_2009.

[BG] A. Beilinson, V. Ginzburg, Wall-crossing functors and $\mathcal{D}$-modules, Represent. Theory 3 (1999), 1-31 (electronic). MR,1659527 (2000d:17007)

[BGS] A. Beilinson, V. Ginzburg, W. Soergel, Koszul duality patterns in representation theory, J. Amer. Math. Soc. 9 (1996), no. 2, 473-527. MR.1322847 (96k:17010)

[CPS] E. Cline, B. Parshall, L. Scott, Duality in highest weight categories, Classical groups and related topics (Beijing, 1987), 7-22, Contemp. Math., 82, Amer. Math. Soc., Providence, RI, 1989. MR982273 (90g:17014)

[Don] S. Donkin, The q-Schur algebra, London Mathematical Society Lecture Note Series, 253. Cambridge University Press, Cambridge, 1998. MR1707336 (2001h:20072)

[DGK] V. V. Deodhar, O. Gabber, V. Kac, Structure of some categories of representations of infinite-dimensional Lie algebras, Adv. in Math. 45 (1982), no. 1, 92-116. MR663417 (83i:17012)

[F] P. Fiebig, Centers and translation functors for the category $\mathcal{O}$ over Kac-Moody algberas, Math. Z. 243 (2003), no. 4, 689-717. MR1974579 (2004c:17051)

[FG] E. Frenkel, D. Gaitsgory, D-modules on the affine grassmannian and representations of affine Kac-Moody algebras, Duke Math. J. 125 (2004), no. 2, 279-327. MR2096675 (2005h:17040)

[GGOR] V. Ginzburg, N. Guay, E. Opdam, R. Rouquier, On the category $\mathcal{O}$ for rational Cherednik algebras, Invent. Math. 154 (2003), no.3, 617-651. MR.2018786 (2005f:20010)

[HTT] R. Hotta, K. Takeuchi, T. Tanisaki, D-modules, perverse sheaves, and representation theory, Progress in Mathematics, 236, Birkhäuser Boston, Inc., 2008. MR2357361 (2008k:32022) 
[J] J. C. Jantzen, Moduln mit einem hohsten Gewicht, Lecture Notes in Math., vol. 750, Springer-Verlag, Berlin and New York, 1980. MR552943 (81m:17011)

[JM] G. James, A. Mathas, A q-analogue of the Jantzen-Schaper theorem, Proc. London Math. Soc. (3) 74 (1997), no. 2, 241-274. MR1425323 (97j:20013)

[Ka] V. Kac, Infinite dimensional Lie algebras, Third edition. Cambridge University Press, Cambridge, 1990. MR.1104219 (92k:17038)

[KV] M. Kapranov, E. Vasserot, Vertex algebras and the formal loop space, Publ. Math. Inst. Hautes Études Sci. No. 100 (2004), 209-269. MR2102701 (2005k:14044)

[K] M. Kashiwara, The flag manifold of Kac-Moody Lie algebra, Algebraic Analysis, Geometry and Number Theory, Johns Hopkins Univ. Press., Baltimore, 1990. MR,1463702 (98i:17030)

[Kas] M. Kashiwara, Equivariant derived category and representation of real semisimple Lie groups, in Representation theory and complex analysis (Lecture Notes in Math., vol. 1931), 137-234, Springer, Berlin, 2008. MR2409699 (2010e:22004)

[KS] M. Kashiwara, P. Shapira, Sheaves on Manifolds, Grundlehren der Mathematischen Wissenschaften, 292, Springer-Verlag, Berlin, 1994. MR.1299726 (95g:58222)

[KT1] M. Kashiwara, T. Tanisaki, Kazhdan-Lusztig conjecture for affine Lie algebras with negative level, Duke Math. J. 77 (1995), no. 1, 21-62. MR1317626 (96j:17016)

[KT2] M. Kashiwara, T. Tanisaki, Parabolic Kazhdan-Lusztig polynomials and Schubert varieties, J. Algebra 249 (2002), no. 2, 306-325. MR.1901161 (2004a:14049)

[KL1] D. Kazhdan, G. Lusztig, Schubert varieties and Poincaré duality, Geometry of the Laplace operator (Proc. Sympos. Pure Math., Univ. Hawaii, Honolulu, Hawaii, 1979), pp. 185-203, Amer. Math. Soc., Providence, R.I., 1980. MR573434 (84g:14054)

[KL2] D. Kazhdan, G. Lusztig, Tensor structures arising from affine Lie algebras I-IV, J. Amer. Math. Soc., 6-7 (1993-1994), 905-947, 949-1011, 335-381, 383-453.

[Ku1] S. Kumar, Toward proof of Lusztig's conjecture concerning negative level representations of affine Lie algebras, J. Algebra 164 (1994), no. 2, 515-527. MR1271251 (95h:17028)

[Ku2] S. Kumar, Kac-Moody groups, their flag varieties and representation theory, Progress in Mathematics, 204. Birkhäuser Boston, Inc., Boston, MR1923198 (2003k:22022)

[L] B. Leclerc, Decomposition numbers and canonical bases, Algebr. Represent. Theory, 3, (2000), no. 3, 277-287. MR1783802 (2001j:17031)

[LT1] B. Leclerc, J-Y. Thibon, Canonical bases of q-deformed Fock spaces, Internat. Math. Res. Notices 1996, no. 9, 447-456. MR1399410 (97h:17023)

[LT2] B. Leclerc, J-Y. Thibon, Littlewood-Richardson coefficients and Kazhdan-Lusztig polynomials, Combinatorial methods in representation theory (Kyoto, 1998), 155-220, Adv. Stud. Pure Math., 28, Kinokuniya, Tokyo, 2000. MR1864481 (2002k:20014)

[M] D. Milicic, Localization and Representation Theory of Reductive Lie Groups, available at http://www.math.utah.edu/ ${ }^{\sim}$ milicic

[PS] B. Parshall, L. Scott, Integral and graded quasi-hereditary algebras, II. with applications to representations of generalized $q$-Schur algebras and algebraic groups, arxiv:0910.0633.

[RT] A. Ram, P. Tingley, Universal Verma modules and the Misra-Miwa Fock space, Int. J. Math. Math. Sci. 2010, Art. ID 326247, 19 pp. MR2753641

[R] R. Rouquier, q-Schur algebras and complex reflection groups, Mosc. Math. J. 8 (2008), no. 1, 119-158, 184. MR2422270 (2010b:20081)

[RW] A. Rocha-Caridi, N. R. Wallach, Projective modules over graded Lie algebras, Math. Z. 180 (1982), no. 2, 151-177. MR661694 (83h:17018)

[Sa] M. Saito, Mixed Hodge modules, Publ. Res. Inst. Sci. 26 (1990), 221-333 MR.1047415 (91m:14014)

[So] W. Soergel, Character formulas for tilting modules over Kac-Moody algberas, Represent. Theory, 2 (1998), 432-448. MR1663141 (2000c:17048)

[St] C. Stroppel, Parabolic category $\mathscr{O}$, perverse sheaves on Grassmannians, Springer fibres and Khovanov homology, Compos. Math. 145 (2009), 954-992. MR2521250 (2011a:17014)

[Su] T. Suzuki, Double affine Hecke algebras, conformal coinvariants and Kostka polynomial, C. R. Math. Acad. Sci. Paris 343 (2006), no. 6, 383-386. MR2259877 (2007f:17038)

[TT] R. W. Thomason, T. Trobaugh, Higher algebraic K-theory of schemes and of derived categories, Grothendieck Festschrift, vol. III, Progr. Math., 88, 247-435, Birkhäuser, 1990. MR.1106918 (92f:19001) 
[VV1] M. Varagnolo, E. Vasserot, On the decomposition matrices of the quantized Schur algebra, Duke Math. J. 100 (1999), no. 2, 267-297. MR1722955 (2001c:17029)

[VV2] M. Varagnolo, E. Vasserot, Cyclotomic double affine Hecke algebras and affine parabolic category $\mathcal{O}$, Adv. Math. 225 (2010), no. 3, 1523-1588. MR.2673739

Département de Mathématiques, Université Paris 7, 175 Rue du Chevaleret, F-75013 PARIS, France

E-mail address: shan@math.jussieu.fr 